\title{
The Near-Saturn Magnetic Field Environment
}

\author{
Author: \\ Ali Haidar Sulaiman \\ Doctoral Supervisor: \\ Professor Michele Dougherty FRS
}

January 2016

Space and Atmospheric Physics Group

Department of Physics

Imperial College London

A thesis submitted in accordance with the requirements for the degree of Doctor of Philosophy and the Diploma of Imperial College London 



\section{Abstract}

Shock waves exist throughout the universe and are fundamental to understanding the nature of collisionless plasmas. The complex coupling between charged particles and electromagnetic fields in plasmas give rise to a whole host of mechanisms for dissipation and heating across shock waves, particularly at high Mach numbers. While ongoing studies have investigated these process extensively both theoretically and via simulations, their observations remain few and far between. This thesis presents a study of very high Mach number shocks in a parameter space that has been poorly explored and identifies reformation using in situ magnetic field observations from the Cassini spacecraft at Saturn's bow shock. This gives an insight into quasiperpendicular shocks across two orders of magnitude in Alfvén Mach number $\left(M_{A}\right)$ and spanning Earth-like to Astrophysical-like regimes. The work here shows evidence for cyclic reformation controlled by specular ion reflection occurring at the predicted timescale of $\sim 0.3 \tau_{c}$, where $\tau_{c}$ is the ion gyroperiod. The relationship between these reformation signatures, magnetic overshoot and variability are also presented.

The final part of this thesis characterises the region downstream of Saturn's bow shock, the magnetosheath. The results show a comprehensive overview of the configuration of the magnetic field in a non-axisymmetric magnetosheath. This non-axisymmetry is revealed to have an impact in the rotation of the magnetic field and is significant enough to influence the magnetic shear at the magnetopause. 


\section{Acknowledgements}

I have received considerable support before and during my $\mathrm{PhD}$. Unfortunately, it is an unspoken rule that the word count in the acknowledgements cannot exceed that of the other chapters and so what follows from here will be a gross understatement.

First and foremost, I wish to thank Prof. Michele Dougherty for giving me the opportunity to embark on $\mathrm{PhD}$ and her unparalleled supervision throughout. In particular, I would like to thank her for always making time to see me, providing such fertile ground to work on and for being willing to sort out many things for me. I have been very fortunate to work with Michele and her team.

I would like to express my sincere gratitude to my advisor, Dr. Adam Masters, for his tireless support, excellent advice and discussions, encouragement during trying times, friendship and keeping me from steering off track.

Furthermore, I would like to thank Prof. David Burgess, Prof. Masaki Fujimoto, Dr. Nick Achilleos and Prof. Steve Schwartz for their brilliant insights and with whom I have had very useful discussions and enjoyed working. My examiners Dr. Jonathan Eastwood, Prof. Jim Slavin and Dr. Ingo Müller-Wodarg for taking their time to read my thesis, conducting a challenging and stimulating viva and offering suggestions that have improved the thesis.

The work in this thesis has been supported financially by STFC, ESA, RAS and NASA, and hosted by Imperial. Many thanks to all.

I have been incredibly lucky to have spent my time at Imperial amongst many new friends, in particular my officemates Simon, for opening my eyes however belatedly to David Bowie, and Jaf, for getting me out of Vegas alive. Josh, Jamie, Flo, Mehdi, Rishi, Steve, Ruth and Luc, thank you for persistently putting up with my excuses for not coming to tea/lunch/pub despite my 1/20 chance of turning up. I'm still adamant they were sound excuses (perhaps apart from "weather-permitting"), but that said, I am already regretting them as I write 
this. I wish you all the utmost success and that sometime in the future I make up for all the times I didn't turn up!

A big part of my $\mathrm{PhD}$ years here were my exhilarating German classes. There I met some of the most inspiring and animated teachers: Dr. Anna Nyburg, Dr. Felicitas Starr-Egger, Dr. Michael Kranert and Herr George Keilbach who passed away recently and will be sadly missed.

This thesis would not have been possible without the treasured companionship of my friends. My undergraduate friends: Tom D, Antoine, Tom C, Navin, Ryan, Axel, Jon, Ahmed, Namir, Yumnah, Max and Shamil. My housemates: Louie, Saul, Veli and Benjy. My school friends: Indie, Kathryn, Scott, Arun, Amar and Briony. My childhood friends: Hussein W, Ali, Maya, Mazen and Hussein F. With each and every one of you, I have my fondest memories, loudest laughs and musical gems both locally and abroad. My partner, Dilsh; her love, reassurance and humour have never ceased to lift me.

Finally and above all, my family for your love, unflagging support and for always believing in me. I owe impossible amounts to my parents, who have now seen me through another chapter in life. My brother and best friend, Tarik. My grandparents, cousins, uncles and aunts, in particular Sherrin who has been a rock all my life.

Thank you. I am indebted to you all. 
For my beloved aunt, Rania 


\section{Declarations}

I hereby declare that this thesis is my own work, it has not been submitted anywhere for any award and all other work has been appropriately referenced. The studies carried out in the course of this degree have led to the publication of the following peer-reviewed scientific papers. Only the first two are presented in this thesis.

Sulaiman, A. H., A. Masters, M. K. Dougherty, D. Burgess, M. Fujimoto, G. B. Hospodarsky (2015), Quasi-perpendicular high Mach number shocks, Phys. Rev. Lett., 115, doi:10.1103/PhysRevLett.115.125001.

Sulaiman, A. H., A. Masters, M. K. Dougherty, X. Jia (2014), The magnetic structure of Saturn's magnetosheath, J. Geophys. Res., 119, doi:10.1002/2014JA020019.

Mistry, R., M. K. Dougherty, A. Masters, A. H. Sulaiman, and E. J. Allen (2014), Separating drivers of Saturnian magnetopause motion, J. Geophys. Res., 119, doi:10.1002/2013JA019489.

The copyright of this thesis rests with the author and is made available under a Creative Commons Attribution Non-Commercial No Derivatives licence. Researchers are free to copy, distribute or transmit the thesis on the condition that they attribute it, that they do not use it for commercial purposes and that they do not alter, transform or build upon it. For any reuse or redistribution, researchers must make clear to others the licence terms of this work. 


\section{Contents}

1. Introduction to Space Plasmas

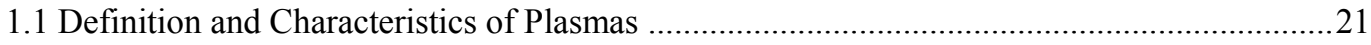

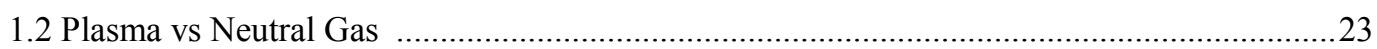

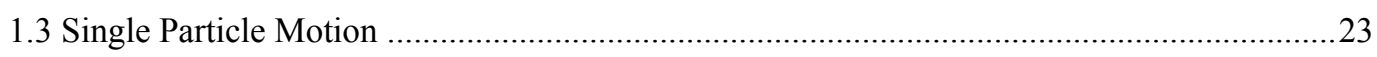

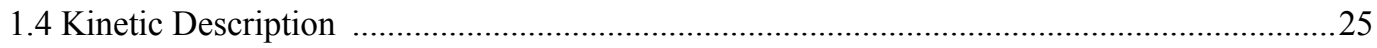

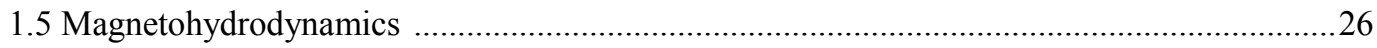

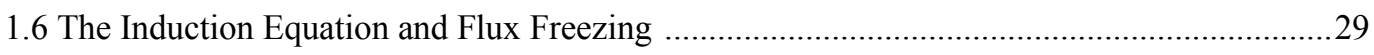

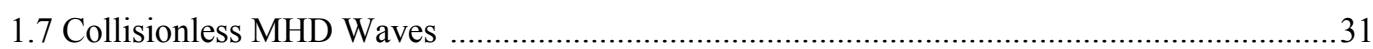

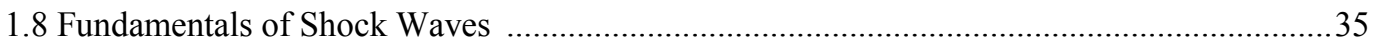

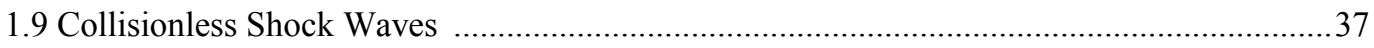

1.9.1 State Variables and Control Parameters ........................................................................ 37

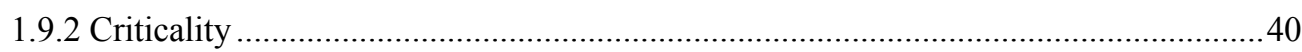

1.9.3 Particle Dynamics and the DC Electric Field ..............................................................

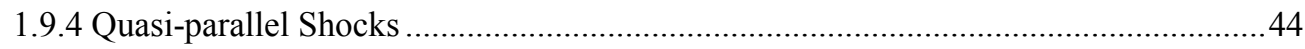

1.10 Applicability of MHD and Kinetic Simulations of Plasmas ..............................................45

2. The Sun-Saturn Connection $\quad 49$

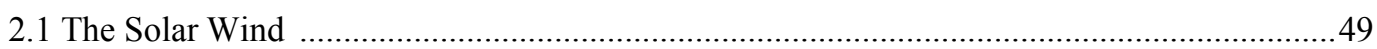

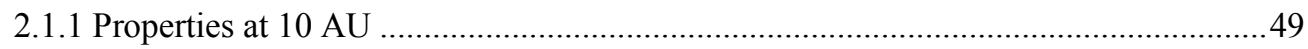

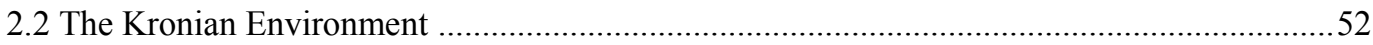

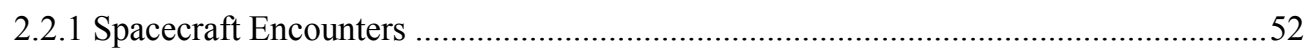

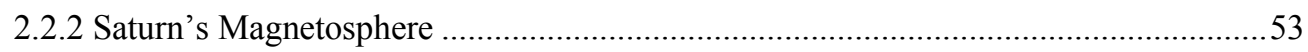

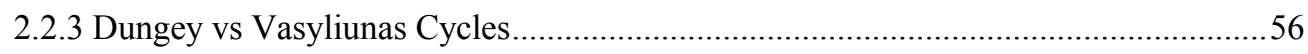

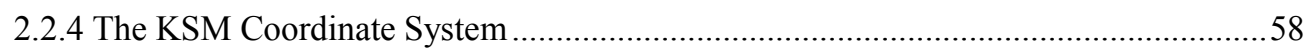

2.2.5 Cassini Observations of Saturn's Dayside Environment ............................................

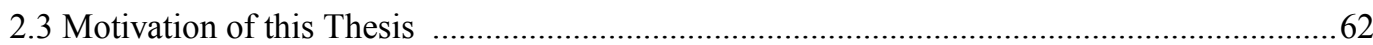

2.3.1 Saturn as a Unique Laboratory for Collisionless Shocks ............................................63

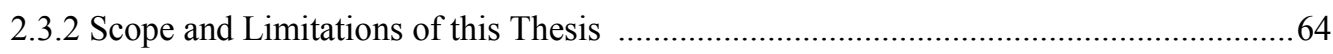

$\begin{array}{lr}\text { 3. Spacecraft and Instrumentation } & 67\end{array}$

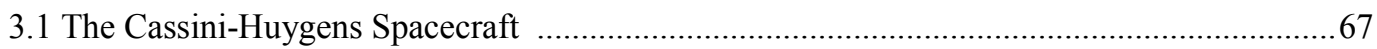




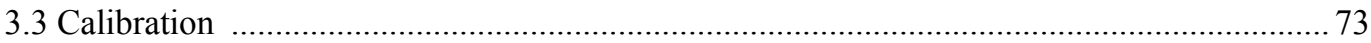

3.4 Cassini Radio and Plasma Wave Science (RPWS) Instrument ........................................ 74

3.5 Cassini Ion Mass Spectrometer (CAPS/IMS) ................................................................... 74

4. Magnetic Field Characterisation of Saturn's Bow Shock $\quad 77$

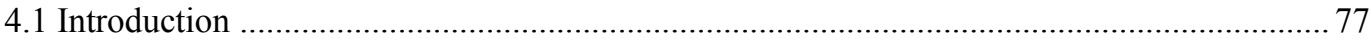

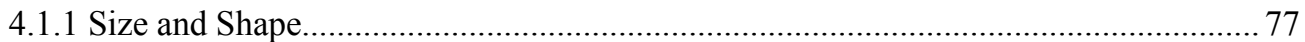

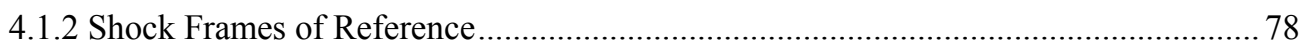

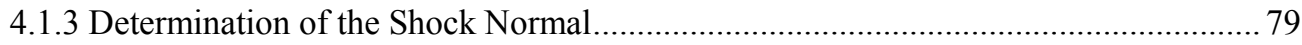

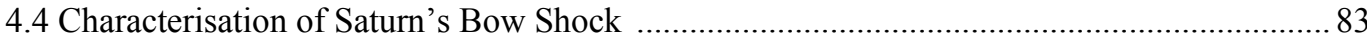

4.4.1 Determination of the Alfvén Mach Number........................................................... 85

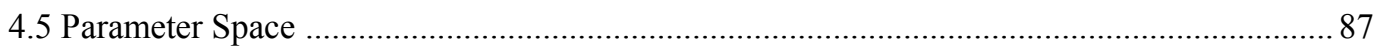

4.6 Shock Relative Overshoot and Variability ................................................................... 91

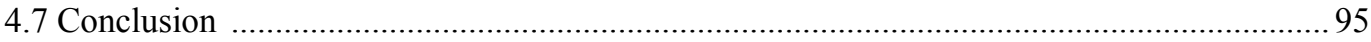

5. Quasi-perpendicular High Mach Number Collisionless Shocks 97

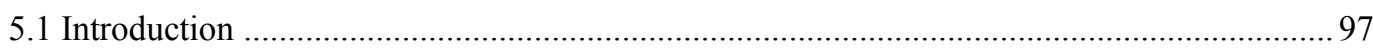

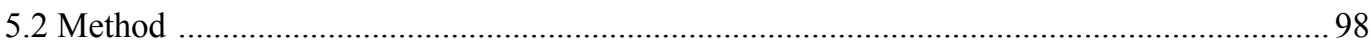

5.2.1 Determination of the Foot Length and Shock Speed ........................................... 99

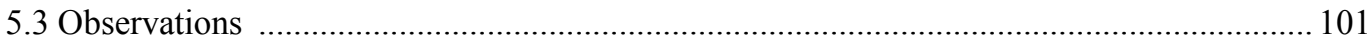

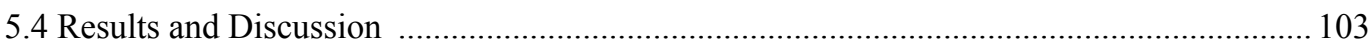

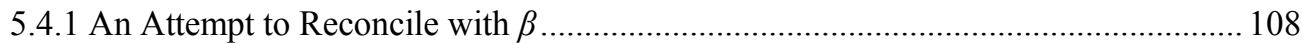

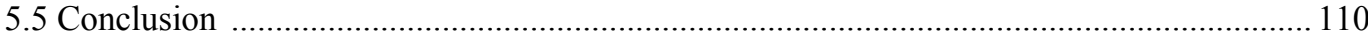

6. The Magnetic Structure of Saturn's Magnetosheath 113

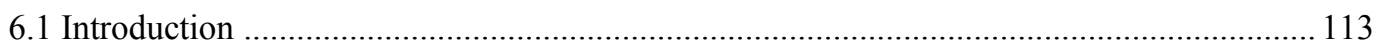

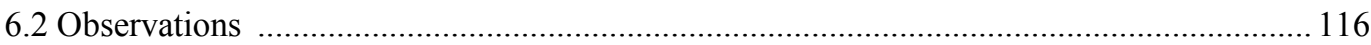

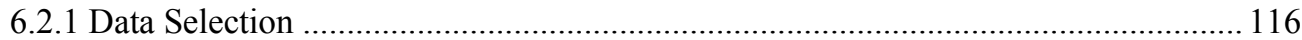

6.2.2 Upstream Conditions and the Overall Configuration of the Magnetic Field in Saturn's

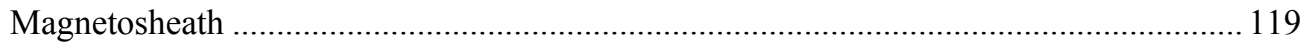

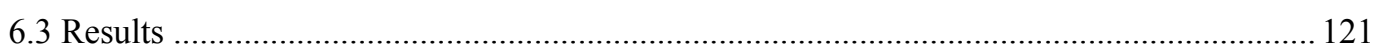

6.3.1 MHD Simulations ..................................................................................... 121

6.3.2 Correspondence between observations and predictive model .............................. 126

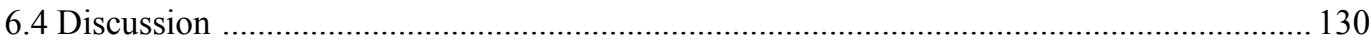

6.Summary and Perspective $\quad 133$

$\begin{array}{ll}\text { Bibliography } & 137\end{array}$ 


\section{List of Figures}

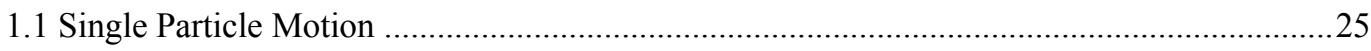

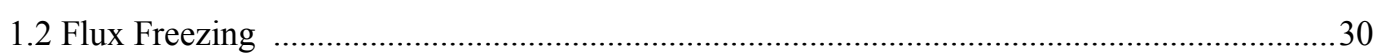

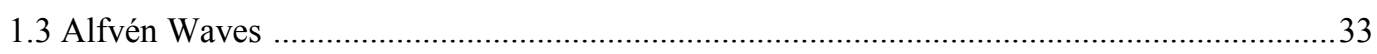

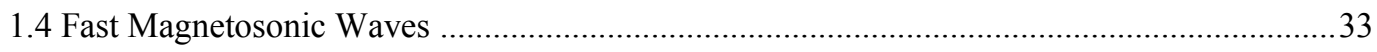

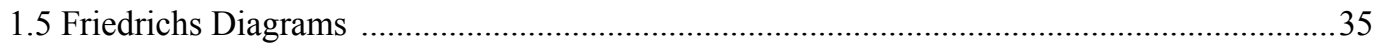

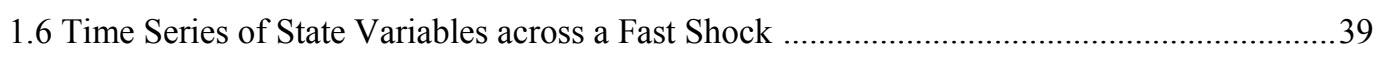

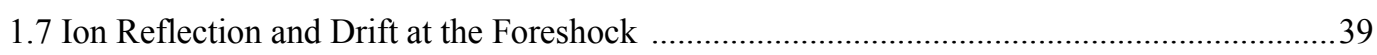

1.8 Hybrid Simulations of a Supercritical Perpendicular Shock .................................................42

1.9 Ion Trajectory and Velocity Space across a Supercritical Shock ............................................43

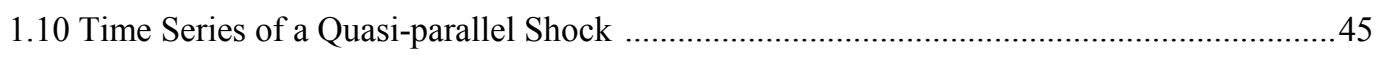

2.1 Solar Wind State Variables with Heliocentric Distance …….................................................50

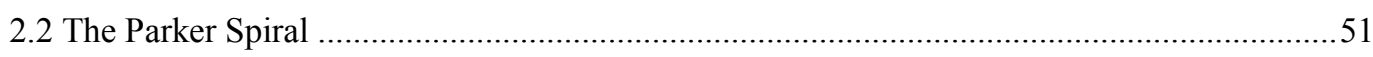

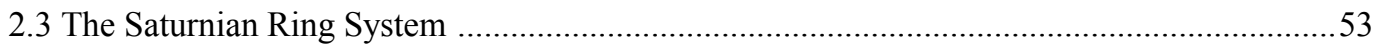

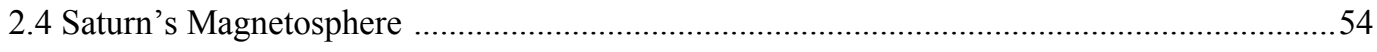

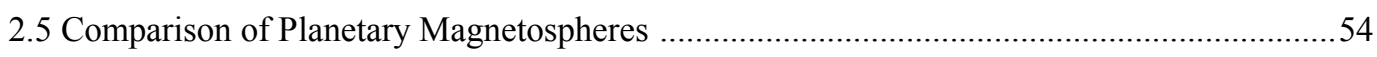

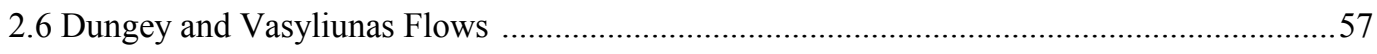

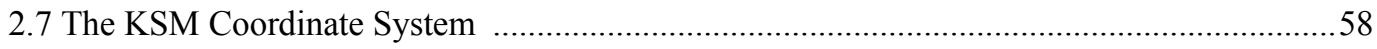

2.8 Time Series of Cassini Traversal of the Magnetosheath ......................................................60

2.9 Global Draping Pattern in the Magnetosheath .................................................................61

2.10 Solar Wind Dimensionless Parameters with Heliocentric Distance ......................................63

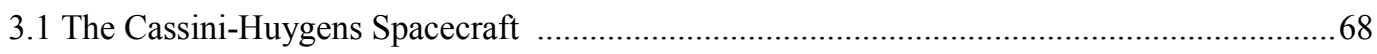

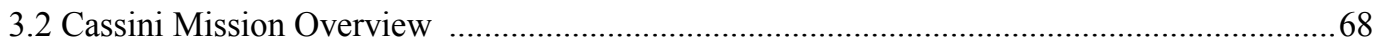

3.3 The Fluxgate Magnetometer ...................................................................................... 71

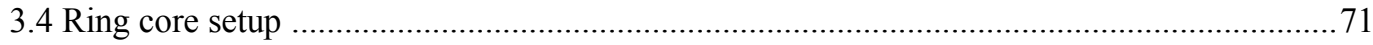

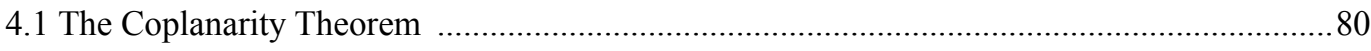

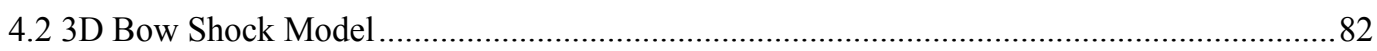




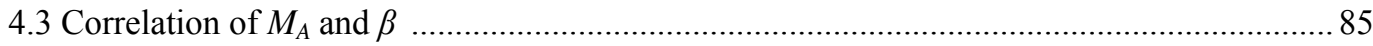

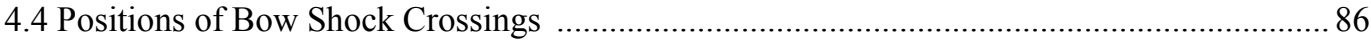

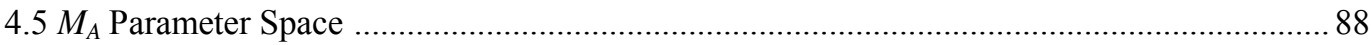

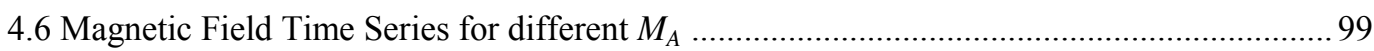

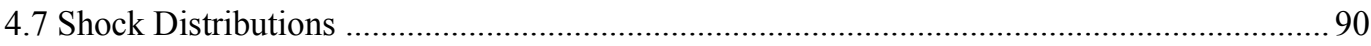

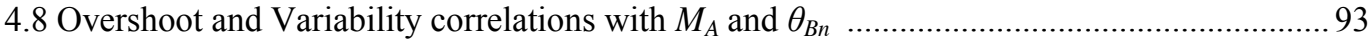

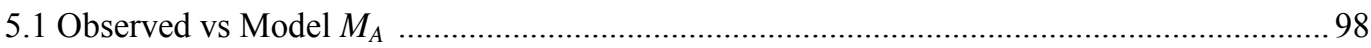

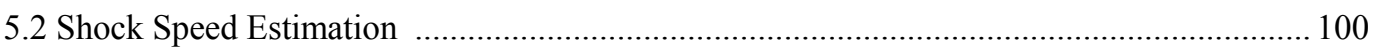

5.3 Magnetic Field Time Series of Reforming Shocks ............................................................ 102

5.4 Normalised Reforming Cycle Periods to Upstream Gyroperiod .......................................... 105

5.5 Trajectories of Specularly Reflected Ions ........................................................................ 106

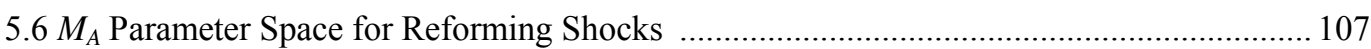

6.1 Z-component of Magnetic Field Upstream of Saturn's Magnetopause …………............... 116

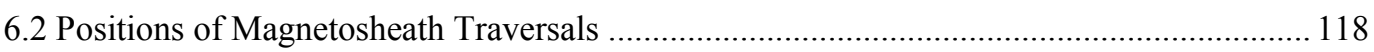

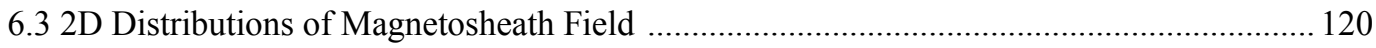

6.4 MHD Simulations for Draping of Field in the Magnetosheath ........................................... 122

6.5 Distribution of Observed Azimuthal Angle vs Local Time ................................................ 124

6.6 Distribution of Observed Meridional Angle vs Latitude .................................................... 125

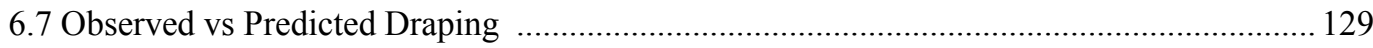

6.8 Conceptual Representation of Polar Flattened Magnetosheath ............................................ 131 


\section{List of Tables}

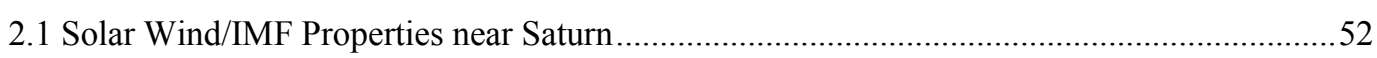

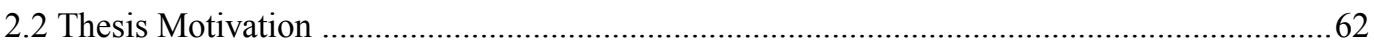

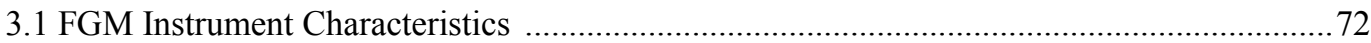

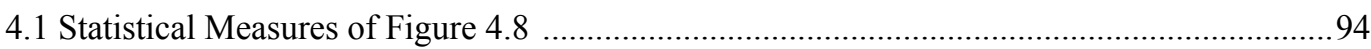

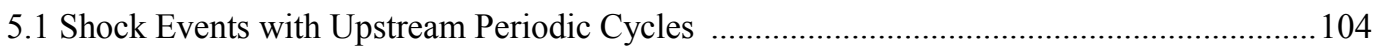

6.1 Upstream Conditions of MHD Simulations ………………................................................123 


\section{Nomenclature}

\begin{tabular}{ll}
$\beta$ & Plasma beta - ratio of thermal and magnetic pressures \\
$\gamma$ & Adiabatic index \\
$\varepsilon_{0}$ & Permittivity of free space \\
$\lambda_{\mathrm{D}}$ & Debye length \\
$\eta$ & Magnetic diffusivity \\
$\mu$ & Mach cone angle \\
$\mu_{0}$ & Permeability of free space \\
$\varphi$ & Spiral angle of interplanetary magnetic field \\
$\Phi$ & Electric potential \\
$\rho$ & Mass density \\
$\rho_{c}$ & Charge density \\
$\rho_{i}$ & Ion mass density \\
$\sigma$ & Conductivity \\
$\tau_{c i}$ & Ion gyroperiod \\
$\theta_{B n}$ & Magnetic contact angle \\
$\omega$ & Angular frequency \\
$\omega_{p e}$ & Electron plasma angular frequency \\
$\omega_{p i}$ & Ion plasma angular frequency \\
$\Omega_{c i}$ & Ion cyclotron frequency \\
ACE & Advanced Composition Explorer \\
AU & Astronomical unit \\
$\boldsymbol{B}_{0}$ & Mean magnetic field \\
$\mathrm{BATS}-\mathrm{R}-\mathrm{US}$ & Block-Adaptive-Tree-Solarwind-Roe-Upwind-Scheme MHD model \\
$\mathrm{CAPS}-\mathrm{IMS}$ & Cassini Ion Mass Spectrometer \\
$c_{s}$ & Speed of sound \\
$d$ (subscript) & Downstream \\
\hline
\end{tabular}




\begin{tabular}{|c|c|}
\hline $\boldsymbol{E}$ & Electric field \\
\hline$e$ & Electric charge \\
\hline FGM & Fluxgate Magnetometer \\
\hline$f_{p e}$ & Electron plasma frequency \\
\hline IMF & Interplanetary Magnetic Field \\
\hline$j$ & Current density \\
\hline KSM & Kronocentric Solar Magnetic coordinate system \\
\hline $\boldsymbol{k}$ & Wave vector \\
\hline$k_{B}$ & Boltzmann constant \\
\hline $\mathrm{L}$ & Length scale \\
\hline $\mathrm{LT}$ & Local Time \\
\hline MAG & Magnetometer \\
\hline MHD & Magnetohydrodynamics \\
\hline$M$ & Magnetic moment \\
\hline$M$ & Mach number \\
\hline$M_{A}$ & Aflvén Mach number \\
\hline$M_{c}$ & Critical Mach number \\
\hline$M_{f}$ & Fast magnetosonic Mach number \\
\hline$M_{s}$ & Sonic Mach number \\
\hline$m_{e}$ & Electron rest mass \\
\hline$n$ & Normal vector to bow shock \\
\hline$n$ & Number density (electrons $n_{e}$; ions $n_{i}$ ) \\
\hline$p$ & Pressure \\
\hline$P_{B}$ & Magnetic pressure \\
\hline$P_{d y n}$ & Dynamic pressure \\
\hline$P_{e}$ & Electron pressure \\
\hline$P_{t h}$ & Thermal pressure \\
\hline$q$ & Electric charge \\
\hline RPWS & Radio and Plasma Wave Instrument \\
\hline ROA & Relative Overshoot Amplitude \\
\hline$R_{m}$ & Magnetic Reynolds number \\
\hline$R_{S}$ & Saturn radius \\
\hline
\end{tabular}




$\begin{array}{ll}R_{S N} & \text { Subsolar distance } \\ r(\theta) & \begin{array}{l}\text { Planetocentric radial distance to bow shock as a function of cone } \\ \text { angle }\end{array} \\ r_{c} & \text { Radius of gyration } \\ \text { SOI } & \text { Saturn Orbital Insertion } \\ T_{e} & \text { Electron temperature } \\ T_{i} & \text { Ion temperature } \\ \text { UTC } & \text { Coordinate Universal Time } \\ \text { u (subscript) } & \text { Upstream } \\ u & \text { Flow speed } \\ \mathbf{v} & \text { Particle speed } \\ \boldsymbol{V} & \text { Bulk flow speed } \\ v_{A} & \text { Alfvén speed } \\ v_{f} & \text { Fast magnetosonic speed }\end{array}$




\section{Introduction to Space Plasmas}

Plasmas are ionised gases in which atoms have dissociated into positively charged ions and negatively charged electrons and, to an extent, can move independently from one another. More than $99 \%$ of the visible Universe is estimated to be in the plasma state, thus making it the most important of the four fundamental states of matter. This chapter introduces the ways of mathematically treating plasma and lays the foundation for the work in this thesis. Most of the background information here can be found in introductory textbooks [e.g. Boyd and Sanderson, 2003].

\subsection{Definition and Characteristics of Plasmas}

The Sun, like any main-sequence star, is a source of radiation strong enough to strip electrons off atoms to form ions. The resulting collection of charged particles is a plasma; a state of matter that fills most of the visible Universe. The solar wind is a stream of plasma remnants originating from the Sun's upper atmosphere and pervading the solar system. The net radial motion of the flow is set up by the large pressure gradient between the solar corona and interplanetary space overcoming the Sun's gravitational pull [e.g. Hundhausen, 1995].

The solar wind comprises nearly equal numbers of electrons and ions. These ions are largely in the form of ionised hydrogen (protons) with some doubly-ionised helium and heavier ions. Overall the number densities of the species are such that charge neutrality is maintained. This property, termed quasineutrality, is fundamental to the treatment of plasmas and the basis upon which one of the characteristic length scales, the Debye length $\lambda_{D}$, is introduced. This length is derived from the effect of Debye shielding [Debye and Hückel, 1923] which arises when a negative test charge is placed in a homogenous plasma. The 
response of the displaced neighbouring electrons and ions produces a polarisation charge that acts to shield the plasma from the test charge. The associated length over which the shielding occurs is given by

$$
\lambda_{D}=\left(\frac{\varepsilon_{0} k_{B} T_{e}}{n_{e} e^{2}}\right)^{\frac{1}{2}}
$$

where $T_{e}$ is the electron temperature and $n_{e}$ is the electron number density. At 10 $\mathrm{AU}\left(1 \mathrm{AU}=1.5 \times 10^{8} \mathrm{~km}\right)$, typical values for $k_{B} T_{e}$ and $n_{e}$ are $\sim 1 \mathrm{eV}$ [Slavin et al., 1985] and $\sim 0.05 \mathrm{~cm}^{-3}$ [Crary et al., 2005] respectively yielding $\lambda_{\mathrm{D}} \sim 30 \mathrm{~m}$. This length is much larger than a spacecraft and thus allows true point measurements [Kivelson, 1995].

A collective interaction in plasmas can be triggered when the electrons are displaced relative to the ions. This separation generates an electrostatic force between the species which acts to restore charge neutrality. Since the electrons have inertia, the response of the system is harmonic with a natural (angular) frequency, $\omega_{p e}$, of the oscillations [Tonks and Langmuir, 1929] derived from Newton's second law and given by:

$$
\omega_{p e}=\left(\frac{n_{e} e^{2}}{\varepsilon_{0} m_{e}}\right)^{\frac{1}{2}}
$$

This quantity is extremely useful in determining the number density of electrons (and ion density by assuming quasi-neutrality) from frequency measurements. A simpler form of the equation is given by:

$$
f_{p e}(H z)=8980 \sqrt{n_{e}\left(\mathrm{~cm}^{-3}\right)}
$$


There exists an equivalent ion plasma frequency, $\omega_{p i}$, which is lower due to the ions' higher inertia. These oscillations are non-propagating and dispersionless, i.e. $c \neq c(k)$, in the limit of a cold plasma where $c$ is the wave speed. In warm plasmas where the thermal temperatures become important, they lead to propagating Langmuir waves.

\subsection{Plasma vs Neutral Gas}

This thesis concerns the solar wind as a supersonic flow and most of the physical problems are therefore addressed using a fluid treatment of plasmas. It is important at this early stage to introduce the distinctions between "everyday" neutral gases and plasmas. Plasmas, like all fluids, exhibit a collective behaviour and its state can be described by macroscopic kinematic and thermodynamic properties. The distinction comes in the nature of the particle interactions within the fluid. The presence of charged particles in a plasma means that the fluid is conductive and therefore responds to, as well as changes, electromagnetic fields. The solar wind, a collisionless plasma, is hot and tenuous with a mean free path of $\sim 1$ AU and thus cannot rely on sufficiently frequent collisions to maintain thermodynamic equilibrium [Baumjohann and Treumann, 1997]. The charged particles instead communicate via complex long-range and bi-directional interactions with the fields. This unique nature of coupling allows information, such as energy changes, to be conveyed across the fluid domain and the bulk properties are adjusted accordingly.

\subsection{Single Particle Motion}

Since the solar wind is supersonic (a term loosely used here and explained in more depth later), the presence of obstacles such as planets and comets give rise to shock waves. It is immediately obvious that in the absence of collisions, the necessary dissipation must be achieved via alternative means unique to collisionless plasmas. Particle trajectories at shock fronts play a major role in contributing to this and it is thus instructive to develop an understanding on how particles respond singly to a background of electromagnetic fields. This approach is the simplest treatment of plasmas. 
For a particle of mass $m$ and charge $q$ moving at a non-relativistic velocity $\mathbf{v}$, the equation of motion in a uniform magnetic field $\boldsymbol{B}$ and in the presence of an external electric field $\boldsymbol{E}$ is given by:

$$
m \frac{d \mathbf{v}}{d t}=q(\mathbf{E}+\mathbf{v} \times \mathbf{B})
$$

where the RHS of the equation is the Lorentz force acting on the particle. The velocity vector can be decomposed into parallel and perpendicular components to the magnetic field, i.e. $\mathbf{v}=\mathbf{v}_{\|}+\mathbf{v}_{\perp}$. It can be shown in the absence of an external electric field $\mathbf{v}_{\|}$is constant while $\mathbf{v}_{\perp}$ changes only direction but not magnitude. The centripetal acceleration sets the particle in motion following a circular path around the magnetic field line with the sense of motion depending on the charge state (see Figure 1.1). The radius of gyration is given by

$$
r_{c}=\frac{\mathrm{v}_{\perp}}{\Omega_{c}}=\frac{m \mathrm{v}_{\perp}}{|q| B}
$$

The particle thus travels a distance $2 \pi r_{c}$ at speed $\mathrm{v}_{\perp}$ in one gyration and it follows that the (angular) gyrofrequency, another natural characteristic frequency, is given by:

$$
\Omega_{c}=\frac{2 \pi}{\tau_{c}}=\frac{q B}{m}
$$

where $\tau_{c}$ is the gyroperiod. The combined motion of a charged particle moving at a uniform speed along a magnetic field line and the gyration around that field line is a helix of constant pitch. 


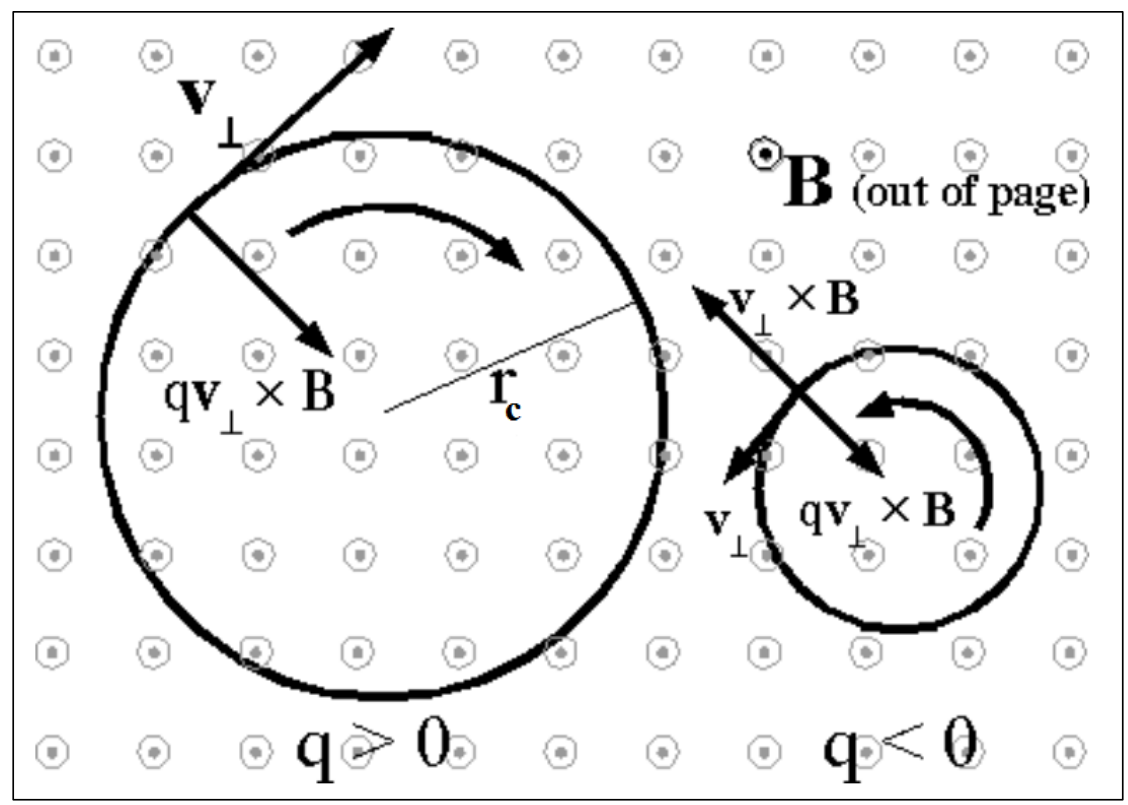

Figure 1.1.: Gyrations of charged particles induced only by a uniform magnetic field $\mathbf{B}$. The Lorentz force acts towards the centre of the gyration with the size of the radius attributed to the mass of particle and the sense - larger $r_{c}$ for heavier proton and smaller $r_{c}$ for lighter electron. The sense of gyration is attributed to the charge state - left-handed for proton and right-handed electron. Figure taken from Schwartz et al. (2002).

\subsection{Kinetic Description}

In certain cases, it is instructive to understand plasmas collectively in a microscopic level that is not within the scope (or interest) of the macroscopic fluid description. This microscopic detail would require the additional information of the particle velocities within a fluid element, since fluid variables in the conservation equations are only functions (and derivatives) of position $\boldsymbol{r}$ and time $t$. Thus, instead of beginning with the density of particles $n(\boldsymbol{r}, t)$, a six-dimensional distribution function $f(\boldsymbol{r}, \boldsymbol{v}, t)$ can be introduced which essentially gives the numbers of particles for a given position $\boldsymbol{r}$ with velocity $\boldsymbol{v}$ at time $t$.

Provided there is no production or loss of particles within a control volume, the distribution function $f$ satisfies the continuity equation. The total number of particles of a species in the volume is given by 


$$
N=\int d^{3} \boldsymbol{r} \int d^{3} \boldsymbol{v} f(\boldsymbol{r}, \boldsymbol{v}, t)
$$

The equation of motion governs the evolution of a phase-space volume along a trajectory defined by $(\boldsymbol{v}, \boldsymbol{a})$, where $\boldsymbol{a}=\boldsymbol{F} / \boldsymbol{m}$ is the acceleration of the volume set up by a Lorentz force $\boldsymbol{F}$ a location $(\boldsymbol{r}, \boldsymbol{v})$. In the absence of collisions, this takes the form

$$
\frac{\partial f}{\partial t}+\boldsymbol{v} \cdot \nabla_{r} f+\frac{\boldsymbol{F}}{m} \cdot \nabla_{v} f=0
$$

The RHS is non-zero if a time-dependent collision term is estimated. This form of the equation is the simplest of the kinetic equation of plasma known as the Vlasov equation [Vlasov, 1968]. This approach of statistically treating plasmas as phase-space volumes greatly simplifies the problem of specifying each of the particles' six dimensions at any given time. There is yet enough information to allow for kinetic phenomena such as wave-particle interactions, otherwise overlooked by MHD.

Macroscopic variables can be approximated by taking moments of the distribution function to yield a number density, bulk velocity and thermal pressure tensor. This approach is valid over a hydrodynamic length scale which is much greater than the particle gyroradius. This gives rise to the concept of a fluid element, characterised by a macroscopic state and from which the fluid approximation of a plasma developed.

\subsection{Magnetohydrodynamics}

The magnetohydrodynamic (MHD) approach treats the plasma as a single conducting fluid thereby obeying the conservation equations of fluid mechanics with appropriate modifications made to account for electromagnetic forces via 
Maxwell's equations [Gurnett and Bhattacharjee, 2005]. The latter set of equations is given by

$$
\text { Poisson's equation: } \quad \nabla \cdot \boldsymbol{E}=\frac{\rho_{c}}{\varepsilon_{0}}
$$

Gauss's law for magnetism:

$$
\nabla \cdot \boldsymbol{B}=0
$$

$$
\text { Faraday's law: } \quad \nabla \times \boldsymbol{E}=-\frac{\partial \boldsymbol{B}}{\partial t}
$$

$$
\text { Ampére-Maxwell law: } \quad \nabla \times \boldsymbol{B}=\mu_{0}\left(\boldsymbol{j}+\varepsilon_{0} \frac{\partial \boldsymbol{E}}{\partial t}\right)
$$

For a MHD fluid limit, two modifications can be made to simplify the algebra in Maxwell's equations. For a single fluid treatment, the Poisson equation can be ignored. Furthermore, the displacement current (i.e. time-dependent term in the $\boldsymbol{\nabla} \times \boldsymbol{B}$ ) equation can also be neglected for a non-relativistic scenario (i.e. $V \ll c$, where $c$ is the speed of light in a vacuum).

The charge-neutral fluid is appropriately described, over a length scale $L \gg \lambda_{D}$, time scale $\tau \gg \Omega_{c}{ }^{-1}$ and speed $V \ll c$, by a mass density $\rho=n\left(m_{i}+m_{e}\right)$, a bulk velocity $\boldsymbol{v}=n\left(m_{i} \boldsymbol{v}_{i}+m_{e} \boldsymbol{v}_{e}\right) / \rho$ and an isotropic thermal pressure $P_{t h}=P_{i}+P_{e}$. The principal differences from the traditional Navier-Stokes equations are the addition of the electromagnetic $\boldsymbol{j} \times \boldsymbol{B}$ force (per unit volume) in the equation of motion and the neglect of the viscous $\mu \nabla^{2} V$ force by virtue of non-collisionality (here $\mu$ is the dynamic viscosity) as well as gravitational and other body forces. The conservation equations of mass and momentum are closed by a constitutive relation of the fluid state variables in the form of an adiabatic equation of state with a ratio of specific heats $\gamma=5 / 3$. Embedded in this are a number of thermodynamic assumptions; mainly that the effect of Ohmic heating $(\boldsymbol{j} \cdot \boldsymbol{E})$ is not important and the pressure is isotropic. Lastly, an Ohm's law describes the conducting properties of the plasma, completing the set. These equations consider an inertial frame of a control volume of plasma, combining the time-dependent changes of the fluid parameters with the convective changes due to spatial gradients. They hold for all species and are given by: 
Mass: $\quad \frac{\partial \rho}{\partial t}+\nabla \cdot(\rho \boldsymbol{V})=0$

Momentum:

$$
\rho\left(\frac{\partial \boldsymbol{V}}{\partial t}+(\boldsymbol{V} \cdot \nabla) \boldsymbol{V}\right)=-\nabla P_{t h}+\boldsymbol{j} \times \boldsymbol{B}
$$

Equation of state:

$$
\left(\frac{\partial}{\partial t}+\boldsymbol{V} \cdot \nabla\right)\left(\frac{p}{\rho^{\gamma}}\right)=0
$$

Ohm's law: $\quad \boldsymbol{E}+(\boldsymbol{V} \times \boldsymbol{B})=\frac{\boldsymbol{j}}{\sigma}+\frac{\boldsymbol{j} \times \boldsymbol{B}}{n e}-\frac{1}{n e} \nabla P_{e}+\frac{m_{e}}{n e^{2}} \frac{d \boldsymbol{J}}{d t}$

Here, $\boldsymbol{V}$ is the bulk motion of the plasma fluid. The RHS of Ohm's law is composed of a $\boldsymbol{j} / \sigma$ resistive term (where $\sigma$ is the conductivity), a $\boldsymbol{j} \times \boldsymbol{B}$ Hall term due to the presence of a magnetic field and a pressure gradient term which drives the lighter electrons, separating them from the ions and consequently setting up an electric field to restore neutrality. A generally small electron inertial term (last term in Equation 1.8d) is often neglected. A special case known as ideal MHD considers the limit where the conductivity is high $(\sigma \rightarrow \infty)$ leading to an electric field that is only motional i.e. $\boldsymbol{E}=-\boldsymbol{V} \times \boldsymbol{B}$.

The electromagnetic $\boldsymbol{j} \times \boldsymbol{B}$ force from the momentum equation can be shown to have two components by manipulating Ampére's law [Kivelson, 1995] as follows

$$
\boldsymbol{j} \times \boldsymbol{B}=\frac{1}{\mu_{0}}(\nabla \times \boldsymbol{B}) \times \boldsymbol{B}=-\nabla_{\perp}\left(\frac{B^{2}}{2 \mu_{0}}\right)+\frac{1}{\mu_{0}}(\boldsymbol{B} \cdot \nabla) \boldsymbol{B}
$$

By drawing an analogy between Equations $1.8 \mathrm{~b}$ and 1.9 , it can be seen from the pressure gradient term that the (perpendicular) pressure contribution from the magnetic field is given by $P_{B}=B^{2} / 2 \mu_{0}$. The second term acts to straighten a magnetic field line under (axial) tension. Using the pressure contribution from the kinetic energy of the plasma's constituent particles, $P_{t h}$, we can express a fundamental and widely-used ratio of the two pressures as: 


$$
\beta \equiv \frac{P_{t h}}{P_{B}}=\frac{P_{t h}}{B^{2} / 2 \mu_{0}}
$$

which is a measure of the relative importance of the kinetic and magnetic forces exerted on a plasma thus determining its behaviour.

\subsection{The Induction Equation and Flux Freezing}

Faraday's law and Ohm's law can be combined and manipulated to eliminate $\mathbf{E}$ and form a governing equation which describes the development of the magnetic field in a plasma with time.

$$
\frac{\partial \boldsymbol{B}}{\partial t}=\nabla \times(\boldsymbol{V} \times \boldsymbol{B})+\eta \nabla^{2} \boldsymbol{B}
$$

The RHS of the equation comprises a convective and a diffusive term (in that order). The former corresponds to the magnetic field lines being intimately connected to the plasma structure and this is the basis upon which the concept of "flux freezing" is introduced. The latter corresponds to the converse when the magnetic field lines can decouple from the plasma structure and diffuse down field gradients. In ideal MHD, the diffusive term vanishes in the limit of an infinite conductivity (where $\eta=1 / \sigma \mu_{0}$ is the magnetic diffusivity) and the induction equation is reduced to only the convective term [Alfvén, 1942; Bondi and Gold, 1950].

A simple scaling analysis can be employed to determine the relative importance of the two terms (as a ratio) in the induction equation. Here the characteristic length is such that $\nabla \sim 1 / L$. This leads to the derivation of the magnetic Reynolds number given by 


$$
R_{m} \equiv\left|\frac{\nabla \times(\boldsymbol{V} \times \boldsymbol{B})}{\eta \nabla^{2} \boldsymbol{B}}\right| \sim \frac{V L}{\eta}
$$

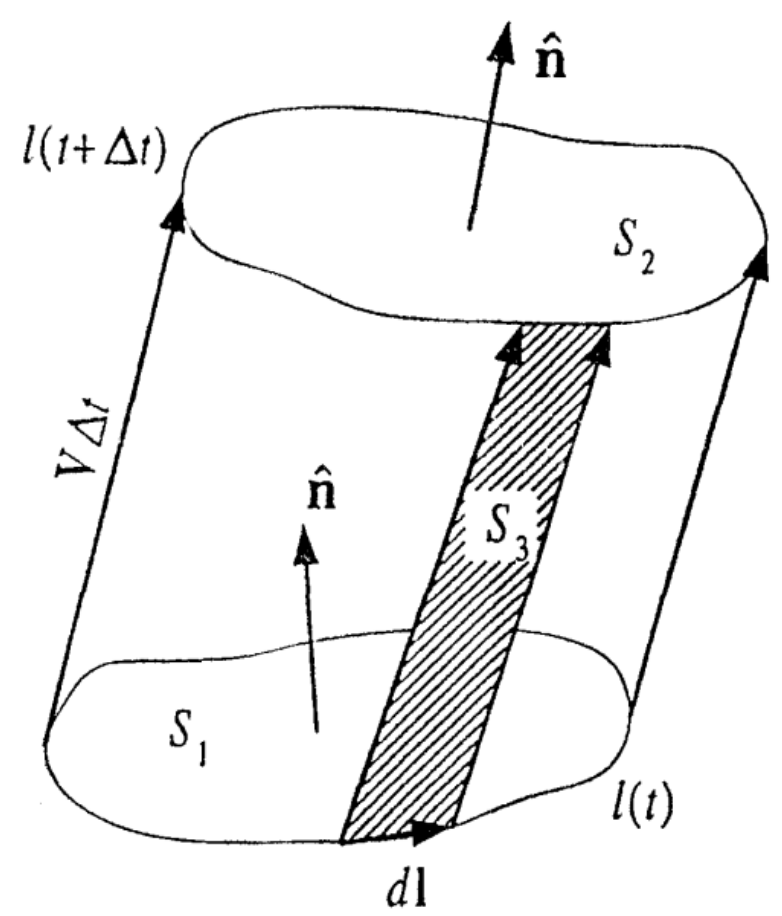

Figure 1.2.: Illustration of "flux freezing". The flux quantity entering the tube at $S_{1}$ remains unchanged at the exit $\mathrm{S}_{2}$. The separation of the field lines changes (linearly) as a result. [Credit: http://www.sp.ph.imperial.ac.uk/ mkd/Handout4.pdf]

For most space plasmas, $R_{m} \gg 1$ (i.e. the ideal MHD approximation holds) and the system can be treated as a perfect conductor into which the field lines are frozen. The case of $R_{m} \ll 1$ is descriptive of very small length scales such as current sheets where field lines can diffuse out of the neighbouring plasma and merge. This reconfiguration of the magnetic topology is known as reconnection and there is an associated transfer from magnetic energy to thermal and kinetic energy resulting in heating and acceleration of the local plasma [Paschmann, 2008].

Now consider the case when the induction equation is only the convective term. Equation 1.13 shows that the rate of change of magnetic flux $\Phi_{B}$ $(\equiv \oint \boldsymbol{B} \cdot \hat{n} d S)$ through a bounded surface is zero. 


$$
\frac{d \Phi_{B}}{d t}=\frac{d}{d t} \iint_{S} \boldsymbol{B} \cdot \hat{n} d S=\iint_{S}\left(\frac{\partial \boldsymbol{B}}{\partial t}-\nabla \times(\boldsymbol{V} \times \boldsymbol{B})\right) \cdot \hat{n} d S=0
$$

Figure 1.2 sketches the necessary components to follow the time derivative of $\Phi_{B}$. The number of magnetic field lines threading an area $S(t)$ bounded by a deformable closed loop $l(t)$ remains unchanged after a time $\Delta t$. This is known as the "frozen-in condition". More intuitively, a fluid element connected by a field line remains connected to the same field line. In space where the flow of plasma is slowed and deflected by an obstacle, the embedded magnetic field lines outside the region continue to convect at the freestream velocity while the field lines near the obstacle are slowed as much as the flow. This leads to the field lines being stretched out behind the obstacle, thereby forming a structure in the form of a "tail". All planets and comets that are obstacles to the solar wind exhibit such structures draped field lines around them.

\subsection{Collisionless MHD Waves}

As touched upon earlier, the conductive nature of a plasma establishes complex interactions between charged particles and electromagnetic fields. For this reason, a plasma can support a variety of wave modes thus allowing information to be propagated by electrostatic and electromagnetic waves. For simplicity and relevance, we shall introduce only magnetohydrodynamic waves.

Wave solutions can be derived from the MHD equations using linear perturbation theory. This involves decomposing the state variables into sums of their background values (as stationary and homogenous, i.e. $\boldsymbol{v}_{\boldsymbol{0}}, \boldsymbol{E}_{\boldsymbol{0}}=0$ ) and space and time dependent small-amplitude fluctuations. The resulting equation is then linearised by dropping higher-order terms. The time and space derivatives are substituted with a frequency $-i \omega$ and a wavenumber $-i k$ index respectively to yield oscillatory solutions. This is by virtue of conveniently assuming the perturbed quantities to vary as $e^{i(k x-\omega t)}$. For "warm" isotropic plasmas (i.e. $\beta$ is not small) 
and assuming no heat flux, the MHD wave equations have non-trivial solutions with a dispersion relation [Kivelson, 1995] given by

$$
\left(\omega^{2}-k^{2} v_{A}^{2} \cos ^{2} \theta_{k B}\right)\left[\omega^{4}-\omega^{2} k^{2}\left(c_{S}^{2}+v_{A}^{2}\right)+k^{4} v_{A}^{2} c_{S}^{2} \cos ^{2} \theta_{k B}\right]=0
$$

where $\theta_{k B}$ is the angle between the wavevector $\boldsymbol{k}$ and the background magnetic field $\boldsymbol{B}$. The dispersion relation has three solutions, namely the fast, intermediate and slow modes. These are:

$$
\begin{gathered}
\text { Fast and Slow: } \frac{\omega^{2}}{k^{2}}=\frac{1}{2}\left(c_{S}^{2}+v_{A}^{2} \pm\left[\left(c_{S}^{2}+v_{A}^{2}\right)^{2}-4 c_{S}^{2} v_{A}^{2} \cos ^{2} \theta_{k B}\right]^{\frac{1}{2}}\right) \\
\text { Intermediate: } \frac{\omega^{2}}{k^{2}}=v_{A}^{2} \cos ^{2} \theta_{k B}
\end{gathered}
$$

Naturally the simplest wave mode in a fluid, the sound speed $c_{s}$, is one characteristic wave speed. This is expressed as

$$
c_{s}=\sqrt{\frac{\gamma p}{\rho}}
$$

The dispersion relation requires that a relation between the frequency and wavenumber must be satisfied for a wave to exist in a plasma. Equation 1.18 shows the dispersion relation followed by another characteristic wave, the Alfvén wave, with a speed $v_{A}$ [Alfvén, 1942].

$$
v_{A}=\frac{B}{\sqrt{\rho \mu_{0}}}
$$

For the case where $\boldsymbol{k}$ and the background magnetic field $\boldsymbol{B}_{\boldsymbol{0}}$ are along the same direction, i.e. $\theta_{k B}=0^{\circ}$ and $\omega^{2}=v_{A} k^{2}$, the Alfvén wave perturbation is trans- 
verse such that the fluctuations $\delta \boldsymbol{v}$ and $\delta \boldsymbol{B}$ are perpendicular to both $\boldsymbol{k}$ and $\boldsymbol{B}_{\boldsymbol{0}}$. The wave is purely magnetic with no plasma compressions, i.e. $\delta \rho, \delta p=0$, and is analogous to a string under tension with the inertia provided by the ions, $\rho=\rho_{i}$.

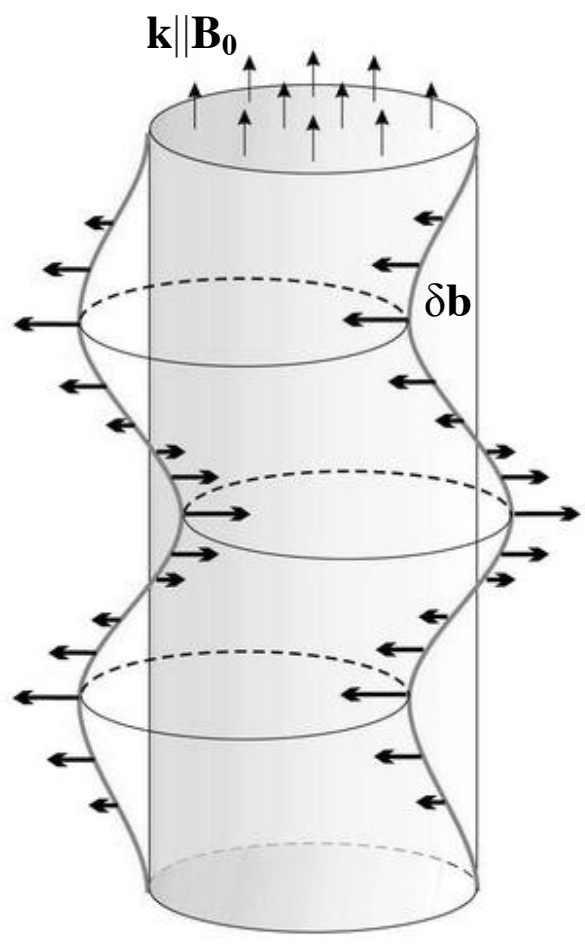

Figure 1.3.: Schematic of Alfvén waves ( $\boldsymbol{k}$ direction) propagating parallel to the background magnetic field $\boldsymbol{B}_{\boldsymbol{0}}$. The magnetic fluctuations $\delta \mathbf{b}$ (and restoring velocity $-\delta \mathbf{v})$ are perpendicular to $\boldsymbol{B}_{0}$ [figure from Morton et al. (2012)].

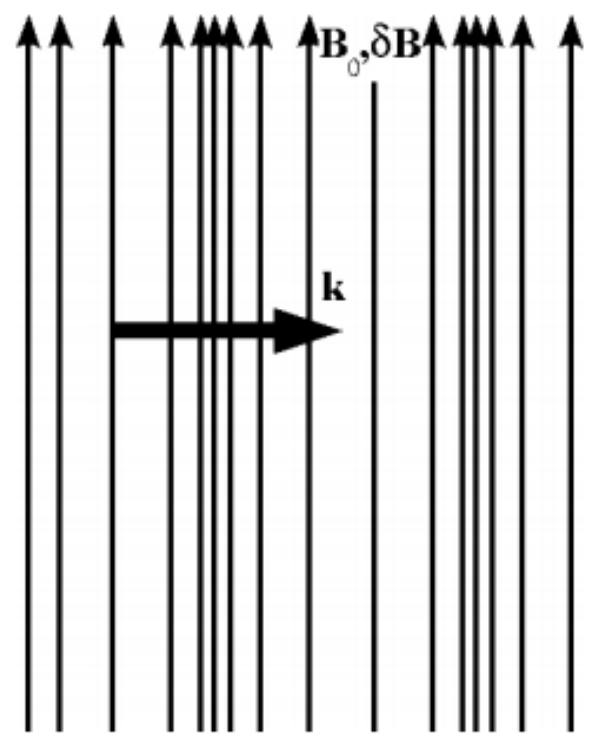

Figure 1.4.: Schematic of fast magnetosonic wave ( $\boldsymbol{k}$ direction) propagating perpendicular to the $\boldsymbol{B}_{\boldsymbol{0}}$. $\delta \mathbf{b}$ are along the magnetic field while $\delta \mathbf{v}$ are perpendicular. The wave is characterised by a series of compressions and rarefactions [figure from

http://www.sp.ph.imperial.ac.uk/ sjs/ APmaster.pdf (page 47)] 
Equation 1.17 represents two solutions combining both the sound and Alfvén speeds to yield a fast (positive sign) and a slow (negative sign) magnetosonic speed. For the case where $\boldsymbol{k}$ and $\boldsymbol{B}_{\boldsymbol{0}}$ are perpendicular to one another, a good assumption for the solar wind near Saturn (as will be explained further), the dispersion relation for the fast mode is reduced to $\omega^{2}=v_{f} k^{2}$ where:

$$
v_{f}=\sqrt{v_{A}+c_{s}}
$$

The fast magnetosonic wave is compressional both in the plasma density and magnetic field. The perturbations of the particle pressure and density are in phase with the perturbations along $\boldsymbol{B}_{\boldsymbol{0}}$. The slow-mode wave, on the other hand, exhibits perturbations of pressure and density that are out of phase with the perturbations along $\boldsymbol{B}_{\boldsymbol{0}}$. The wave motion can occur obliquely with the largest possible value perpendicular to $\boldsymbol{B}_{\boldsymbol{0}}$. The general case for the fast, intermediate and slow wave modes can be illustrated using Friedrichs diagrams (Figure 1.5). These show the variations of the wave modes over a range of $\theta_{k B}$. Starting from the end case $\boldsymbol{k} \| \boldsymbol{B}_{\boldsymbol{0}}$, it can be seen for $v_{A}>c_{s}$, the fast mode coincides with the Alfvén speed and the slow mode with the sound speed, thereby decoupled from the magnetic field. The converse is also true for $v_{A}<c_{s}$. As $\theta_{k B}$ increases, there is a smooth variation in the phase speeds until $\boldsymbol{k} \perp \boldsymbol{B}_{\boldsymbol{0}}$ where the fast mode is $\left(v_{A}+c_{S}\right)^{1 / 2}$ and the other two vanish. Both the fast mode and slow mode shocks are compressive and associated with an increase in entropy. They differ in the tangential component of the magnetic field which decreases across a slow mode shock and increases across a fast mode shock.

Figure 1.5. (following page): Friedrichs diagrams illustrating the phase speeds (normalised to $v_{A}$ ) of the fast, intermediate (shear Alfvénic) and slow wave modes. The background magnetic field is in the vertical direction. A line $(\boldsymbol{k})$ connecting the origin to any point on a given contour has a length proportional to the phase speed of the corresponding wave mode with the angle between $\boldsymbol{k}$ and $\boldsymbol{B}_{\boldsymbol{0}}$ equal to $\theta_{k B}$. The case on the left corresponds to $v_{A}>c_{s}$ and on the right $v_{A}<c_{s}$. 

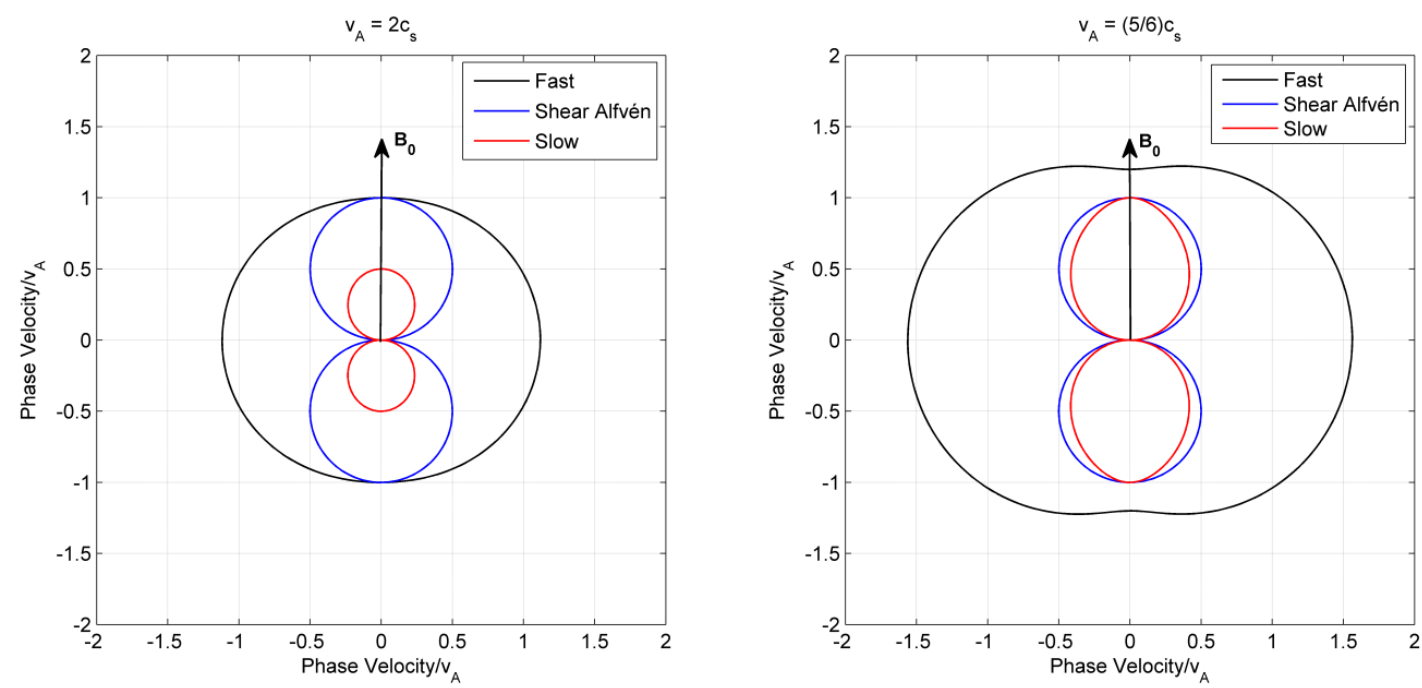

\subsection{Fundamentals of Shock Waves}

A discontinuity is a thin, abrupt transition layer between two equilibrium states of a fluid. Shock waves are a type of discontinuity where mass flows across the transition layer. Unlike the MHD waves in $\S 1.1 .7$, the inherent nonlinear terms in the equations of motions, such as $(\boldsymbol{V} \cdot \boldsymbol{V}) \boldsymbol{V}$, are appreciable and thus retained for their description. This is because the wave steepness scale becomes comparable to the dissipation scale.

In ordinary fluids, the formation of shock waves requires a relative flow between an obstacle and the surrounding fluid. Consider a fixed obstacle immersed in a flowing fluid of speed, $u$. Since the problem is frame invariant, here we shall consider the conventional (Eulerian) frame of reference where the obstacle is stationary. When a parcel of the flow is close enough to the obstacle, it must deflect around it as the conservation laws do not permit piling up of the material. To achieve this in sufficient time, the obstacle conveys information of its presence to the upstream flow. This information is transferred at a characteristic speed - the speed of sound $c_{s}$ - and maintains a constant entropy, as long as the flow is subsonic, i.e. $u<c_{s}$. The entropy assumption is such that $p \rho^{-\gamma}$ is constant and Equation 1.19 is derived from this. 
The arbitrary flow speed has no upper limit, thus there is a transition when the flow can become supersonic, i.e. $u>c_{s}$, and a problem arises when the information about the obstacle's presence cannot propagate to the upstream flow fast enough. As a consequence, this information transfer becomes entropic (and irreversible) through the formation of a shock. The purpose of the shock is to sufficiently and abruptly heat, compress and slow the flow down to a subsonic regime downstream where the timeliness of the information transfer is no longer a problem. In other words, there is a conversion of directed ram energy upstream to random thermal energy downstream with the increase in entropy stemming from the particles' spread in velocity space. The shock is a steepened fast magnetosonic wave and hence the extent of its dissipation to achieve the necessary transition from supersonic to subsonic flows is governed by the conservations of mass, momentum and energy. A set of equations can then be derived, known as the Rankine-Hugoniot (RH) jump conditions, which locally relate the flow states upstream and downstream through these conservation laws. These conditions dictate to a maximum compression, i.e. $\rho_{d} / \rho_{u}$, of 4 for $\gamma=5 / 3$ (where subscripts ' $u$ ' and ' $d$ ' denote the upstream and downstream regions respectively). The temperature rise, however, has no maximum. It is worth noting that the shock's permeability between two regions of different pressures means that the flow properties change from one equilibrium state to another.

The shock has a finite thickness in reality, as opposed to being a perfect discontinuity, within which the dissipation takes place. In ordinary fluids, collisions sufficiently thermalise the flow within the shock yielding a thickness in the order of kinetic scales. This region (and those immediately neighbouring it) are not captured by the fluid description of the RH equations which are only concerned with predicting the fate of the flow state given their initial state and not with the microphysical means by which it is achieved. The values of the flow state parameters entering the $\mathrm{RH}$ equations must therefore be taken from regions far enough from the transition layer so as not to mix in dissipative processes.

Shock waves are characterised by their Mach number, $M$; a ratio of the relative flow speed to a characteristic speed. For ordinary fluids, the sonic Mach number, $M_{s}$ is given by: 


$$
M_{s} \equiv \frac{u}{c_{s}}
$$

where it can easily be seen that $M_{s}>1$ is a necessary condition for shocks to exist. The Mach number is an indicator of a shock's strength; as this increases, the shock must process a larger flow volume per unit time to satisfy the $M_{s}<1$ downstream. Note that the Mach number only concerns the flow along the shock normal, i.e. $u=u_{n}$.

\subsection{Collisionless Shock Waves}

\subsubsection{State Variables and Control Parameters}

The solar wind is essentially collisionless with a particle mean free path of $\sim 1$ AU. It is thus immediately obvious that for (thin) shock waves to form, some other non-local mechanisms must come into play to compensate for the lack of collisions. Nevertheless, the conservation laws must still hold and we will begin by introducing the RH jump conditions for collisionless plasmas which, unlike ordinary fluids, folds in the momentum and energy contributions of electromagnetic fields [Burgess, 1995]. These are:

$$
\begin{aligned}
& {\left[\rho u_{n}\right]=0} \\
& {\left[B_{n}\right]=0} \\
& {\left[\rho u_{n}^{2}+P_{t h}+\frac{B_{t}^{2}}{2 \mu_{0}}\right]=0} \\
& {\left[\rho u_{n} \boldsymbol{u}_{\boldsymbol{t}}-\frac{\boldsymbol{B}_{\boldsymbol{t}} B_{n}}{\mu_{0}}\right]=0} \\
& {\left[\left(\frac{\gamma P_{t h}}{(\gamma-1) \rho}+\frac{u^{2}}{2}\right) \rho u_{n}+\frac{u_{n} B_{t}^{2}}{\mu_{0}}-\frac{B_{n}\left(\boldsymbol{B}_{\boldsymbol{t}} \cdot \boldsymbol{u}_{\boldsymbol{t}}\right)}{\mu_{0}}\right]=0} \\
& {\left[u_{n} \boldsymbol{B}_{\boldsymbol{t}}-B_{n} \boldsymbol{u}\right]=0}
\end{aligned}
$$


Here, $u$ is the flow speed in the shock's rest frame with subscripts ' $n$ ' and ' $t$ ' denoting the components normal and tangential to the shock surface respectively. The other usual variables are those defined earlier. The square brackets "[X]" denote the difference between the parameters downstream and upstream. Equation $1.23 \mathrm{a}$ is derived from the steady state continuity equations and represents the conservation of mass flux across a shock. Equation $1.23 \mathrm{~b}$ represents the conservation of the normal component of the magnetic field by virtue of Gauss's law (Equation 1.7b). Equations $1.23 \mathrm{c}, 1.23 \mathrm{~d}$ and $1.23 \mathrm{e}$ are the steady state conservation of normal momentum, tangential momentum and total energy respectively. Finally Equation 1.23f represents the conservation of tangential electric field which is an extension of the conservations of mass flux and normal component of magnetic field.

For such magnetised plasmas, the strength of the shock is characterised by three Mach numbers associated with each characteristic speed. These are the fast magnetosonic $M_{f}$, Alfvén $M_{A}$, and sonic $M_{s}$ Mach numbers. For example, a fast magentosonic shock forms when the flow of collisionless plasma exceeds the speed at which an obstacle's presence is transmitted upstream along characteristics i.e. fast magnetosonic waves. An additional (geometric) parameter is the contact angle, $\theta_{B n}$, between the shock normal $\widehat{\boldsymbol{n}}$ and the upstream magnetic field vector $\boldsymbol{B}$. This quantity is used to distinguish between two categories of shocks: quasi-parallel and quasi-perpendicular where $0^{\circ} \leq \theta_{B n}<45^{\circ}$ and $45^{\circ}<\theta_{B n} \leq 90^{\circ}$ respectively (see Figure 1.7).

Now that an introduction of collisionless shocks has been given, we can explore the unique (and still open) questions associated with them: How do collective plasma kinetic phenomena serve to provide the required "heating" and irreversibility? Under what conditions do certain mechanisms become important (and others no longer important)? Several irreversible processes have been proposed, namely dissipation, dispersion and reflection, where long-range interactions between the charged particles and self-consistently generated electromagnetic fields result in the necessary RH heating and increased entropy [Treumann, 2009]. The relative importance of these mechanisms depends, at least as widely believed, on the strength of the shock, i.e. the Mach number. 


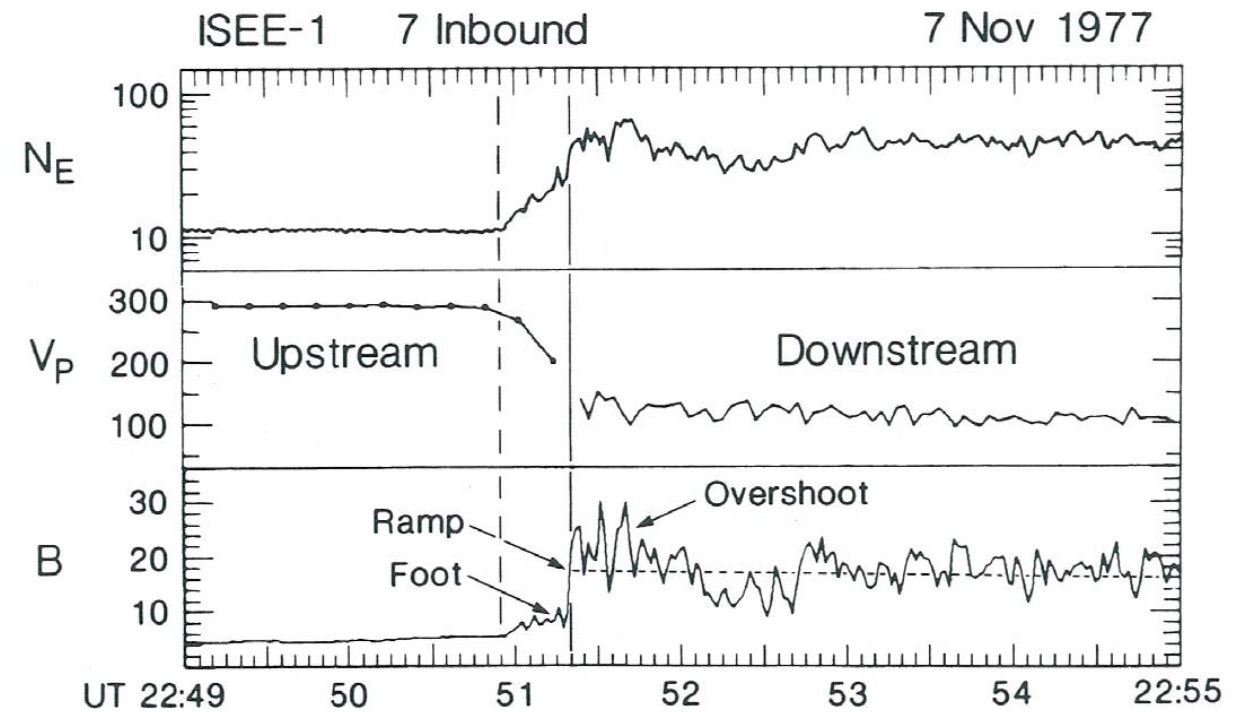

Figure 1.6.: Time series of an inbound (upstream to downstream) ISEE crossing of Earth's bow shock. As predicted by MHD RH equations, there is an increase in electron number density $N_{e}$ and magnetic field $B$ accompanied by a decrease in proton bulk speed $V_{p}$ [Burgess, 1995].
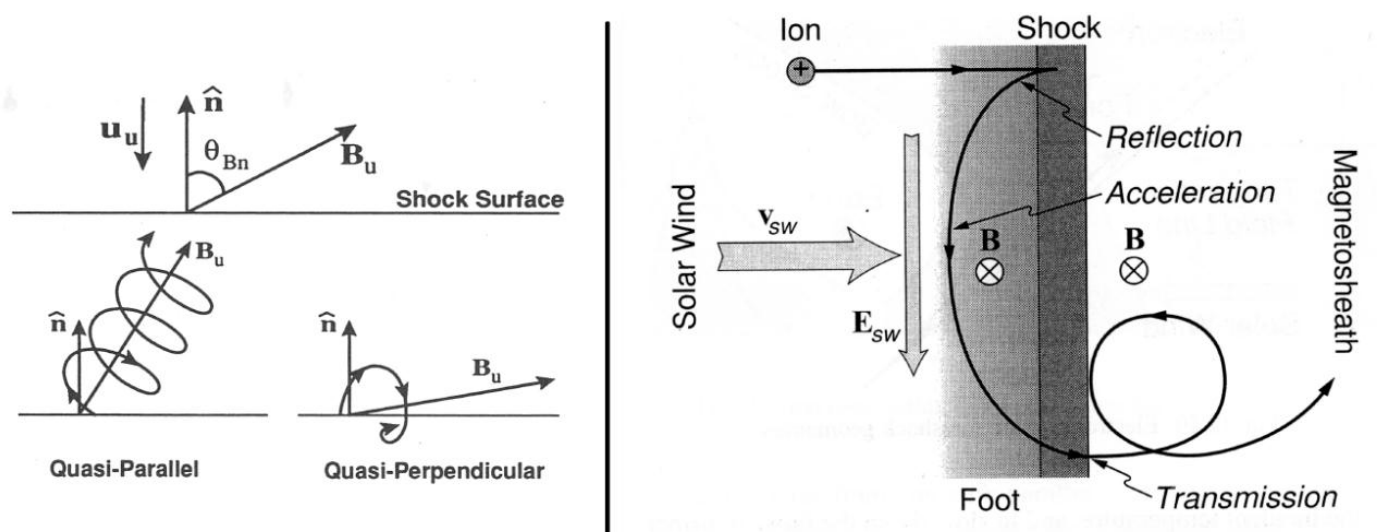

Figure 1.7.: Ion pathways upon encountering a supercritical shock front for quasi-parallel and quasi-perpendicular configurations (left). The drift of reflected ions to the motional electric field (right). 
Figure 1.6 shows one of the earliest observations of an inbound crossing of Earth's bow shock. The changes in bulk parameters (i.e. averages of downstream and upstream) are indeed as predicted by the MHD RH equations. Strictly speaking, the frame dependent parameters (the bulk proton speed $V_{p}$ in this case) are not necessarily numerically consistent with the RH equations since they are in the spacecraft rather than the shock frame. The sense of change, i.e. a decrease, is nevertheless correct. Out of the context of the fluid description, the overshoot is indicative of a supercritical shock - this is further discussed in the next subsection.

\subsubsection{Criticality}

Consider the formation of a fast magnetosonic shock $\left(M_{f}>1\right)$ which is most commonly found in planetary environments. Other classes (e.g. intermediate shocks) can occur not only when the intermediate speed is exceeded by the flow velocity, but also under the additional condition that the fast speed is inhibited in the local medium. This is dependent on the local sound and Alfvén speeds (as illustrated by Figure 1.5), which in turn depends on the local temperature, magnetic field and density. At low Mach numbers (denoted simply as $M$ for generality) just above unity, the shock could be capable of dissipating the required energy entirely through resistivity from "anomalous collisions" owing to the collective interactions between particles and fields. As the arbitrary flow speed continues to increase (or equivalently a change in the thermodynamic properties decrease the characteristic speeds), the Mach number continues to rise accordingly. Inevitably a point will be reached where the shock is incapable of converting an excess of ram energy upstream into thermal energy downstream. This character of the shock, in maintaining the required "heating" solely by anomalous resistivity, is referred to as its criticality [Marshall, 1955]. The largest critical Mach number is $M_{c} \approx 2.8$, depending on $\theta_{B n}$ and $\beta$. Shocks with $M<M_{c}$ are subcritical and $M>M_{c}$ are supercritical; the latter being the most commonly observed in planetary bow shocks, particularly the further the planet is from the Sun. Here, a model beyond the fluid description, namely particle dynamics, must be invoked to compensate for the shortcoming in "heating". This is achieved by means of re- 
flecting a portion of the incoming ions upstream and it can be thought of as the shock decreasing the effective Mach number it is seeing.

\subsubsection{Particle Dynamics and the DC Electric Field}

Ion reflection is a fundamental process in supercritical shocks and their associated signatures are prominent in observations. For quasi-perpendicular shocks, the reflected ions reencounter the shock after their partially-gyrated reflection and are ultimately transmitted downstream with the convected field (see Figure 1.7a). These reflections are manifested as an enhancement just preceding the shock ramp. The feature is known as the foot and its locality corresponds to the magnetic field orientation restricting the ions from escaping far enough upstream. The enhancement in the magnetic field arises from the formation of a current layer by the motional electric field. This electric field acts to transversely drift the reflected ions to a velocity $v_{t}$ as depicted by Figure $1.7 \mathrm{~b}$. The current density in this layer is thus $j_{y} \sim e n_{i, r e l f} v_{t}$ with the corresponding foot magnitude as $B_{z} \sim \mu_{0} j_{y} d_{n}$; where $d_{n}$ is the shock thickness along the normal. Another unique feature is the shock overshoot between the ramp and the downstream field. The enhancement in the magnetic field here can be significantly larger than the RH limit of 4 . Note Equations 1.23a and 1.23f show that for a perfectly perpendicular shock, $\theta_{B n}=90^{\circ}$, the field compresses exactly as much as the flow i.e. $\rho_{d} / \rho_{u}=$ $B_{d} / B_{u}$ [Burgess, 1995]. These kinetic features are typically present in the magnetic field observations of supercritical shock crossings. Figure 1.8 (top) shows the magnetic field profile of an inbound supercritical shock crossing with the kinetic features within and near the transition layer clearly apparent. Hybrid simulations support the invocation of microscopic ion dynamics as shown by the phase space diagrams in Figure 1.8 (bottom). The ions are shown to reverse in the $x$-direction (along the shock normal), indicative of specular reflection at the shock front and a non-zero velocity in the $y$-component (tangential to the shock front) indicates drift motion by virtue of the motional electric field.

The dynamics of particles (both ions and electrons) are controlled by an electric potential within the shock layer. The source of this is a DC electrostatic field set up by both the magnetic field profile and the electron temperature gradi- 
ent, $E \approx-\frac{1}{n e} \nabla P_{e}$, from Ohm's Law (Equation 1.8d). This DC field acts to slow down the incoming flow and to reflect back some fraction of the incident ions [Thomsen et al., 1987]. The reflected ions are accelerated once exposed to the motional electric field before returning back to the shock with enough energy to overcome the potential barrier (since $E=-\nabla \Phi$ ). This cross-shock potential also means that leaked ions downstream are accelerated back into the upstream region. This combination of transmitted and reflected ions leads to a slower net bulk flow speed and larger velocity spread in the downstream region [Schwartz et al., 2013]. As a consequence, the ions experience a rise in their kinetic temperature and are thus "heated" by virtue of the DC field without the need for collisions. Strictly speaking, the increase in kinetic temperature is reversible and the dissipation comes from additional processes, mainly instabilities, which scatter the resultant distributions. The ion instabilities occur at timescales larger than the time spent in the ramp thus ion "heating" extends to the downstream region to complete the dissipation.
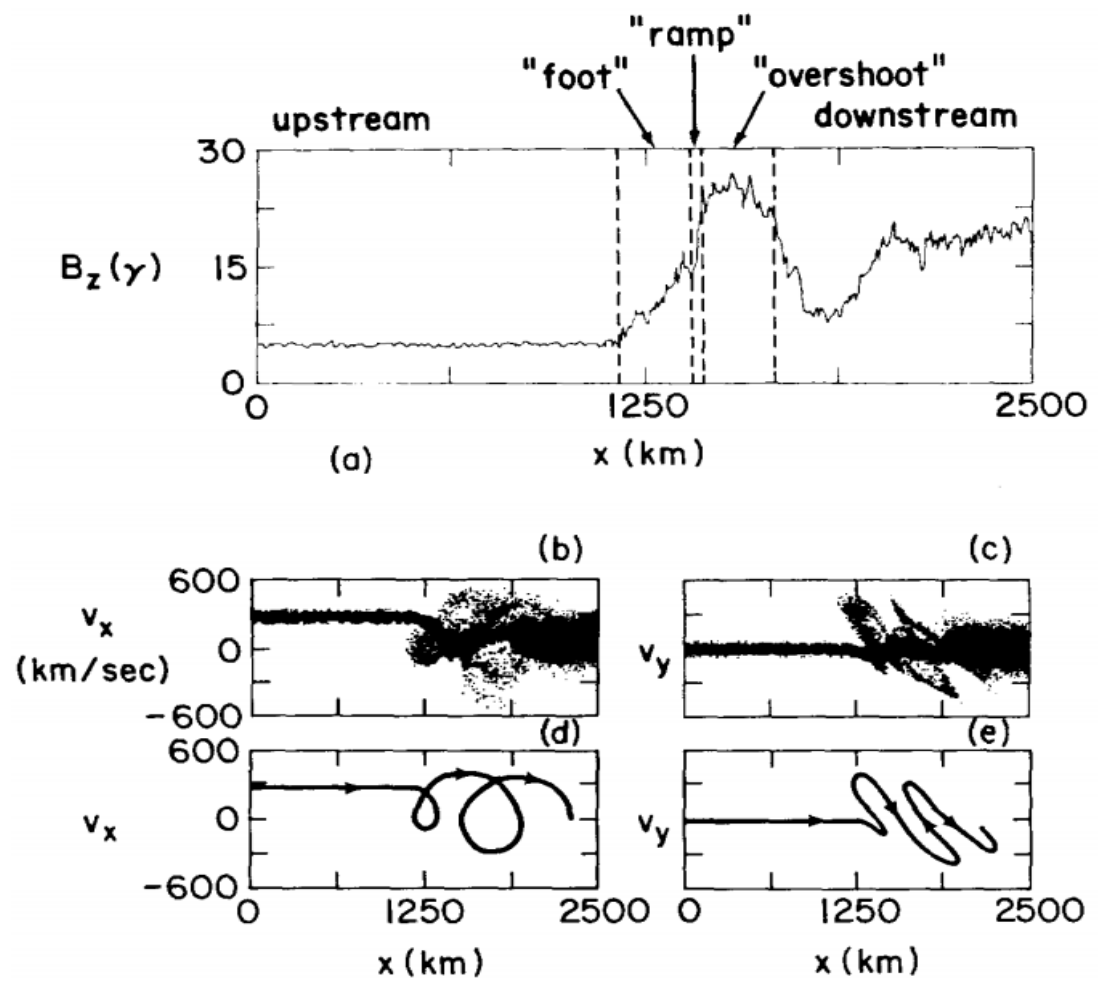

Figure 1.8.: Hybrid simulations of a supercritical perpendicular shock revealing the foot, ramp and overshoot features in the magnetic field profile (top). Simultaneous ion phase space profiles (middle row) and a typical orbit (bottom row) [Wu et al., 1984]. 
This process is more intuitively illustrated in Figure 1.9. The shock is divided into three regions namely upstream, ramp and downstream with a simplified, instantaneous distribution in velocity space corresponding to each region. The filled circle represents the incoming population which is directed in the $x$ direction (note here the $x$-direction is not parallel to the shock normal). This population is decelerated across the shock $\left(1 \rightarrow 1^{\prime}\right)$ and very slightly deflects in the $y$ direction. The empty circles represent the reflected population. Upon reflection, particles instantaneously exhibit almost purely a $v_{y}$ component (3). They are then accelerated in the ramp (2') and into the downstream region with gyration motions instantaneously directed both upstream (2"') and downstream (2"). The decrease in bulk speed and increase in temperature are represented by the average and variance of the velocity distributions respectively.

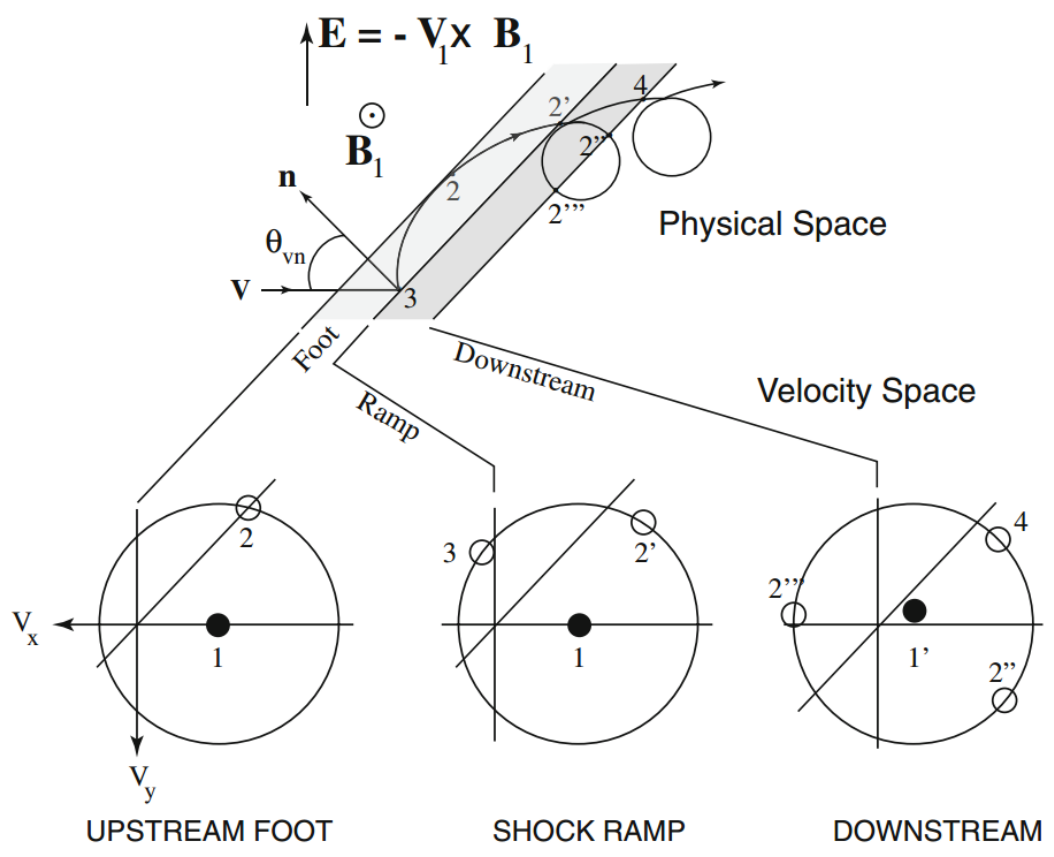

Figure 1.9.: Trajectory (top) and velocity space (bottom) of ions encountering a shock surface. The two dashed lines represent cuts immediately upstream (left) and downstream (right). The latter shows a lower net bulk speed with a larger spread [Sckopke et al., 1983]. 
Conversely, the electrons (which can have thermal speeds greater than the bulk speed) respond to this DC field with the incident population gaining energy and the escaping heated population, originating far downstream, losing energy. While this acceleration/deceleration of the electrons is reversible, the consequence of an inflation in velocity space is a 'hole' that requires filling up by dissipative processes which impose irreversibility [Bale et al., 1998; Balikhin and Gedalin, 1994; Mitchell and Schwartz, 2013]. Overall, the shock dissipation is principally controlled by ion dynamics particularly at higher Mach numbers [Ghavamian et al., 2013], though the reflection process is intimately coupled to the electron heating via the DC electric field as described.

Supercritical shocks, under certain conditions, undergo quasi-periodic reformation i.e. they are not time-stationary. This process is attributed principally to ion dynamics where, at high Mach numbers, the fraction of reflected ions becomes large. The reflected population results in an ion beam accumulating at the foot. This creates a local hole (within a ring-vortex) in the velocity-space ion distribution which triggers scattering of both the incoming and reflected populations. The magnetic field in the hole is depressed relative to the ring-vortex, leading to the formation of a new ramp as the foot magnetic field further increases. At this time, the position of the ramp progresses upstream to the foot and the shock is reformed. The 'fossils' of the ring-vortex can then be recognised in the downstream distribution. This process can be supressed at high $\beta_{i}$ where the high thermal velocity can smear out the hole thus bridging the gap between the incoming and reflected ions [Scholer et al., 2003; Treumann, 2009].

\subsubsection{Quasi-parallel Shocks}

A quasi-parallel shock is defined as having an angle $\theta_{B n}<45^{\circ}$. Figure 1.10 illustrates in situ magnetic field signatures for a typical crossing of a quasiparallel shock for $\theta_{B n}=21^{\circ}$. In contrast to a quasi-perpendicular shock, the crossing is characterized by a transition layer extending from a foreshock region far upstream, excited by wave-particle interactions, to the downstream region [Schwartz and Burgess, 1991]. 


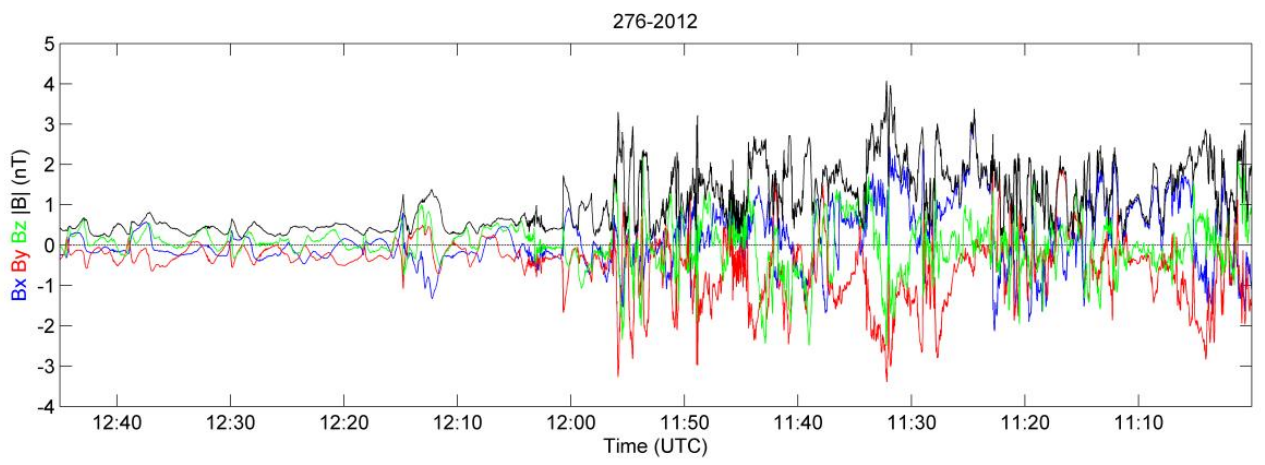

Figure 1.10.: Magnetic field plot of a Saturnian quasi-parallel bow shock crossing. The time series has been reversed to display in the upstream-to-downstream direction.

The foreshock is the region upstream of a shock wave filled with backstreaming particles and is thus detected where the spacecraft is magnetically connected to the shock. For a quasi-perpendicular shock, this is simply the foot region. On the other hand, the foreshock in a quasi-parallel configuration is spatially much broader and the particles in this region are energetic enough to counter the solar wind beam and escape the shock region permanently [Eastwood et al., 2005]. While these backstreaming ions and electrons experience the same $\boldsymbol{E} \times \boldsymbol{B}$ drift, the difference in their momenta results in velocity dispersion. This leads to the formation of two regions: an electron foreshock extending farthest away from the quasi-parallel shock - since they are affected less by the $\boldsymbol{E} \times \boldsymbol{B}$ drift owing to their higher energy - and an ion foreshock closer to the shock.

\subsection{Applicability of MHD and Kinetic Simulations of Plasmas}

Simulations play a crucial role in complementing observations thus providing a deeper insight into addressing questions concerning collisionless plasmas, and can also point to where additional observations are needed [Winske and Omidi, 1996].

Both MHD and kinetic approaches are widely used in understanding space physics plasmas; the choice of one over another being a matter of scale. MHD simulations are appropriate for investigating large scale (i.e. much greater than 
gyroscales) phenomena, for example the global patterns of the solar wind flow and embedded magnetic field draping around a planetary magnetosphere [Gombosi et al., 2002]. Here, the simulations can be useful in understanding flowobstacle interactions of varying degrees of asymmetry and compressibility (such as the magnetospheres of Earth and Saturn), to name a few [e.g. Jia et al., (2012)]. On the other hand, kinetic simulations are appropriate when changes occur on gyroscales and the retention of single-particle dynamics is necessary. Planetary bow shocks are a relevant site for processes occurring at these scales [e.g. Leroy et al., 1981].

Two commonly used techniques are Particle-in-cell (PIC) and Hybrid. In PIC simulations, both ions and electrons are represented as finite phase-space volumes in their respective distribution functions [Winske and Omidi, 1996]. The evolution, of the macroparticles' positions and velocities at each time step, is progressed using field information from the grid; the new particle information then modifies the field. While the macroparticle approach alleviates the computational cost by reducing the number of particles to be treated, the treatment is still relatively computationally intensive and further approximations need to be made [Scholer and Burgess, 2007]. One way is by reducing the dimensionality of the system (e.g. $2.5 \mathrm{D}$ which simulates momentum in $3 \mathrm{D}$ but restricts space to $2 \mathrm{D}$ ); another is the choice of ratios artificially less than their physical values (e.g. the ion to electron mass ration $m_{i} / m_{e}$ of 1836 and electron plasma to gyrofrequencies of $\omega_{p e} / \Omega_{p e}$ of $\left.\sim 100-200\right)$ since the simulation cost is nonlinearly proportional to the ratios.

Hybrid simulations are similar to PIC in that ions are treated as macroparticles, but the electrons are described as an inertialess, adiabatic fluid maintaining neutrality. Consequently, processes on scales of $\omega_{p e}$ or $\Omega_{p e}$ cannot be captured and this is compensated by some finite resistivity to suppress gradient steepening to these electron scales where the hybrid simulation does not apply [Burgess, 1987]. This treatment is, for these reasons, better suited to processes principally driven by ion dynamics. The advantage of hybrid simulations is that higher dimensionality is more achievable. Another cheaper version is the hybrid-fluid model in which the ion motion is represented by fluid parameters. 
An alternative approach uses the Vlasov equations to directly model the six-dimensional ion distribution function while treating the electrons as a fluid. This is known as the hybrid-Vlasov approach and is substantially more computationally expensive than the PIC approach. The advantage of the hybrid-Vlasov approach is its ability to produce noise-free ion distribution functions comparable to those obtained by spacecraft measurements [e.g. Pokhotelov et al. (2013)]. 


\section{The Sun-Saturn Connection}

This chapter summarises the large-scale structure and average properties of the solar wind in the near-Saturn environment. An introduction to Saturn's magnetosphere will follow, emphasising on its uniqueness in parameter range and from which the motivation of this thesis is derived.

\subsection{The Solar Wind}

The solar wind is the continuous flow of plasma flowing radially from the Sun and occupying the solar system. The high Reynolds number in the solar corona results in the solar wind and the Sun's magnetic field convecting in unison (see §1.5) to form the interplanetary magnetic field (IMF). The bubble enclosing the solar wind is called the heliosphere and extends far beyond the solar system until the pressure is balanced by interstellar plasma. This boundary is called the heliopause and in situ observations of interstellar plasma by Voyager 1 have recently been reported thus suggesting that the boundary has been crossed [Gurnett et al., 2013].

\subsubsection{Properties at 10 AU}

As the solar wind expands away from the Sun, the flow properties evolve accordingly as a function of heliocentric distance. Some of the earliest direct measurements were made by Gringauz et al. (1951) and Snyder and Neugebauer (1966). Over decades, dozens of spacecraft have been able to characterise these properties from inside the orbit of Mercury to far beyond the solar system with the most extensive and robust observations being made in the near-Earth environment. This variation of flow states therefore has implications for a unique interaction between the solar wind and each planet. 

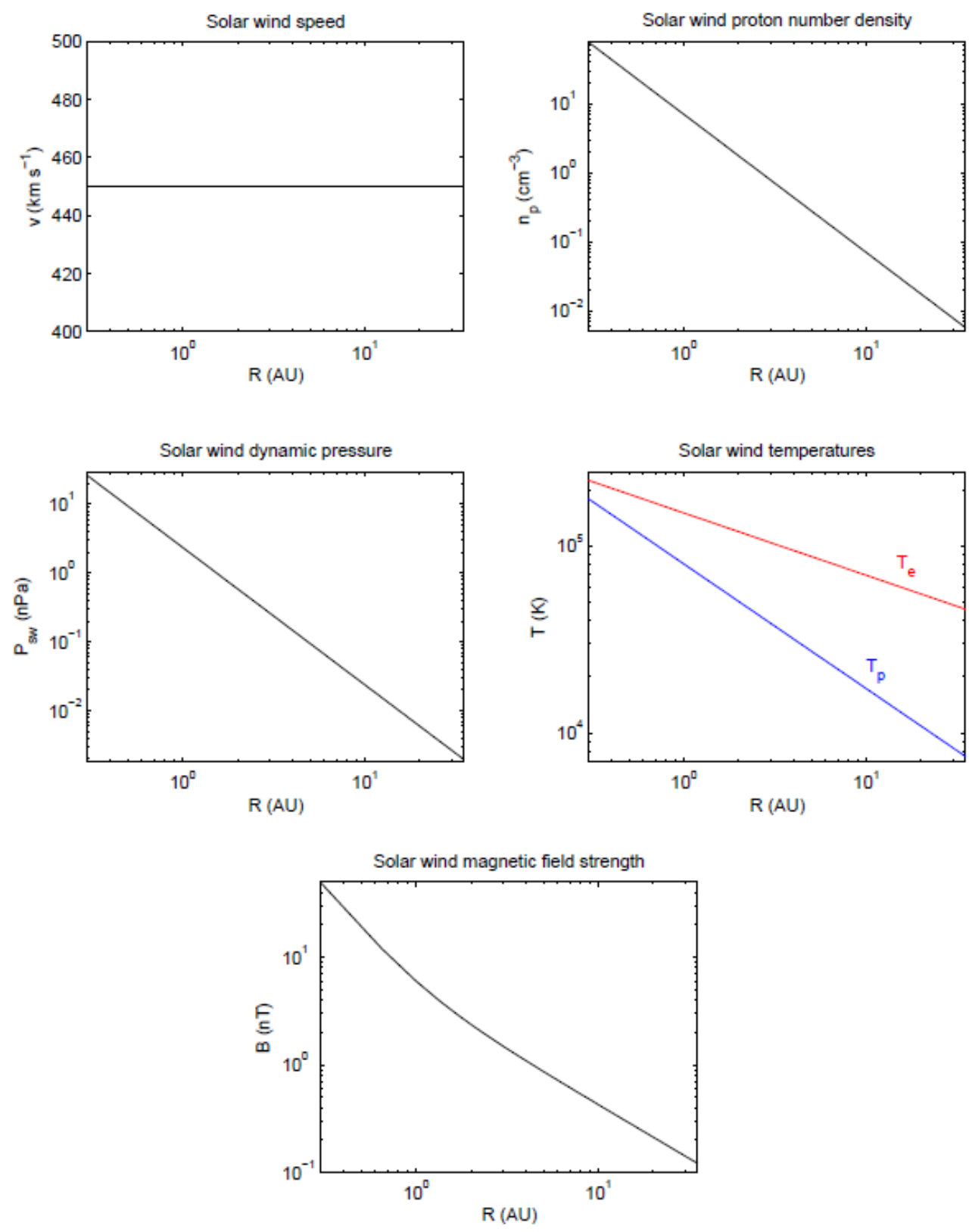

Figure 2.1.: Solar wind state variables as a function of heliocentric distance. The locations $1 \mathrm{AU}$ and $10 \mathrm{AU}$ are of Earth and Saturn respectively.

Figure 2.1 illustrates the spatial dependence of the flow parameters highlighting the Earth and Saturn at 1 AU and 10 AU respectively (1 Astronomical Unit $\left.(A U)=1.5 \times 10^{11} \mathrm{~m}\right)$. Table 1.1 presents the average properties of some of these parameters as measured by the Cassini-Huygens spacecraft (hereafter referred to as Cassini). The plots take typical values at Earth and assumes a scaling 
density as $r^{-2}$, proton and electron temperatures as $r^{-1}$ and $r^{-2}$ respectively, magnetic field strength as $r^{-1}\left(r^{-2}+1\right)^{1 / 2}$, and the solar wind speed as constant [Russell et al., (1982)]. In addition, the IMF has a prevailing global structure across the heliosphere which is controlled by the solar rotation. The combination of this rotation and the radially flowing solar wind gives an average distribution of the equatorial IMF in the shape of an Archimedean spiral, known as the Parker spiral [Parker, 1958]. Within this plane (see Figure 2.2), the Parker spiral makes an average angle of $\sim 45^{\circ}$ near Earth which becomes increasingly azimuthal to $\sim 87^{\circ}$ at Saturn. This has significant consequences on the dynamics of planetary shocks (e.g. $\theta_{B n}$ for the spatial extent of foreshocks, particle acceleration, hot flow anomalies etc.) and magnetospheres (e.g. the magnetic shear angle for reconnection at the magnetopause). The IMF is separated into two regions of magnetic field lines pointing away and towards the Sun by a heliospheric current sheet.

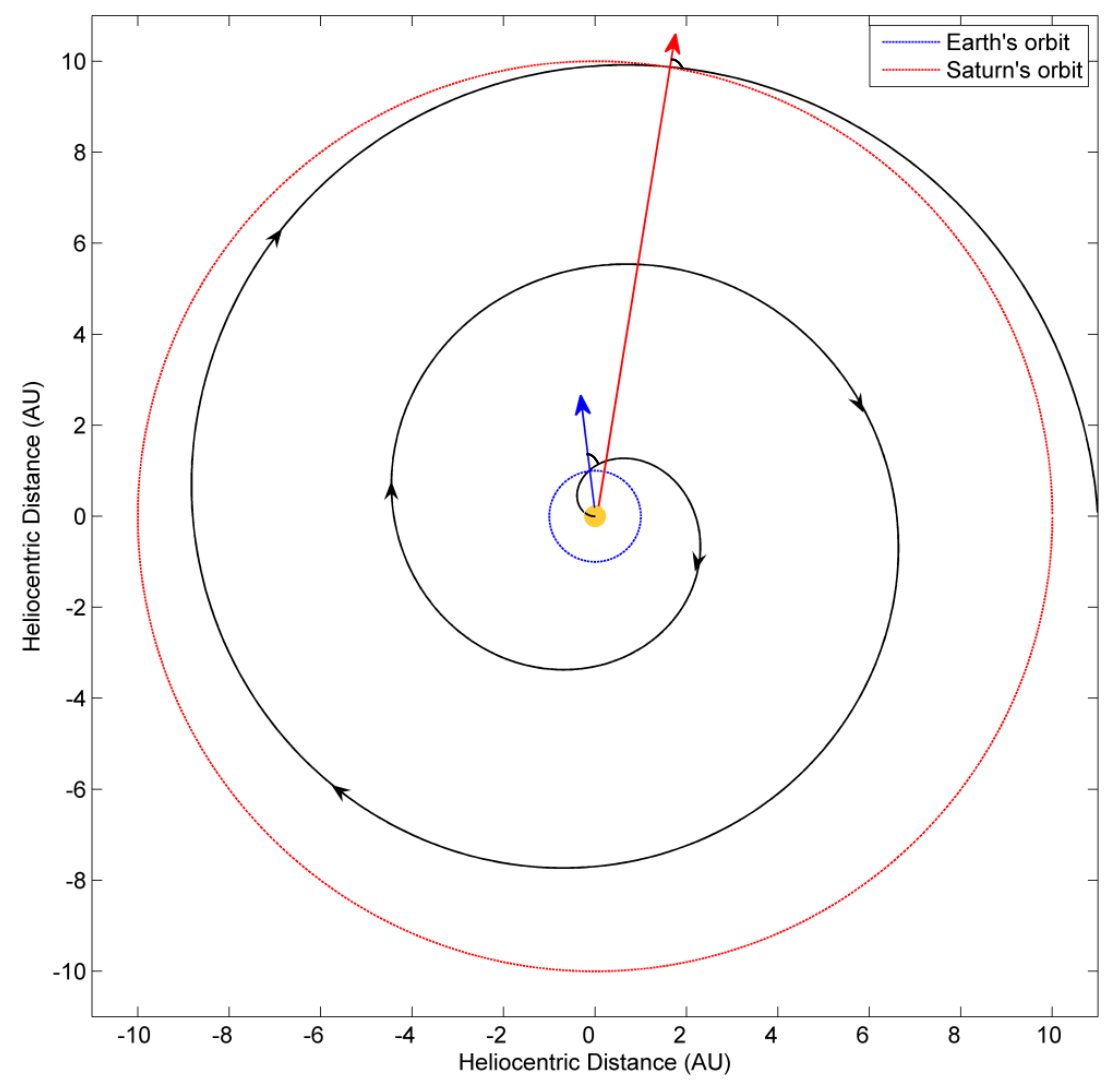

Figure 2.2.: Projection of the Parker spiral on the Sun's equatorial plane. The blue ring indicates $1 \mathrm{AU}$ and the red ring $10 \mathrm{AU}$. The radial arrows indicate the direction of plasma ejected from the Sun. It can be seen that the angle between the radian vector and magnetic field becomes increasing azimuthal (clockwise) with increasing heliocentric distance. 


\begin{tabular}{lll}
\hline Parameter & Typical Range/Value & \\
\hline Proton Number Density & $0.001-1 \mathrm{~cm}^{-3}$ & Crary et al. $(2005)$ \\
Proton (Radial) Velocity & $450-600 \mathrm{~km} / \mathrm{s}$ & Crary et al. $(2005)$ \\
Magnetic Field Strength & $0.1-3 \mathrm{nT}$ & Jackman et al. $(2008)$ \\
Parker Spiral Angle & $87^{\circ}$ & Jackman et al. $(2008)$ \\
\hline
\end{tabular}

Table 1.1.: Solar wind/IMF properties near Saturn

\subsection{The Kronian Environment}

\subsubsection{Spacecraft Encounters}

Saturn is the second largest planet in the solar system after Jupiter. Its equatorial radius $R_{s}$ is roughly ten times that of Earth's at $60,268 \mathrm{~km}$. Orbiting at $\sim 10 \mathrm{AU}$, it is the sixth furthest from the Sun and rotates about its axis once every $10 \mathrm{~h} 45 \mathrm{~m}$ (though still an open question). This axis is inclined by approximately $26^{\circ}$ to the ecliptic plane. Like all other planets, Saturn has a large number of natural satellites with 62 confirmed to date. It has the most extensive and sophisticated ring system with recent studies showing evidence of interactions between the ring and atmosphere [e.g. O'Donoghue et al., 2013]. Tidal activities have also been reported to play a role in the formation of moonlets [e.g. Murray et al., 2008] dubbing Saturn's rings as a "mini solar system". Figure 2.3 depicts the extent of Saturn's ring system. This consists of a visible, dense set of inner rings $(\mathrm{A}, \mathrm{B}, \mathrm{C}$ and $\mathrm{D})$ and a diffuse set of outer rings ( $\mathrm{E}$ and $\mathrm{F})$; the latter are distributed between the orbits of many satellites. Arguably the most peculiar feature of Saturn is the near-perfect alignment between its rotation and magnetic moment axes. This axisymmetry appears to violate Cowling's theorem, which states that a dynamo cannot be maintained when $\mathbf{M} \| \boldsymbol{\Omega}$, thereby prompting longstanding debates on the sustainability of its intrinsic magnetic field [e.g. Stevenson, 1982].

Saturn has been explored by four spacecraft: Pioneer 11 in 1979 [Acuna and Ness, 1980], Voyager 1 in 1980 [Ness et al., 1981], Voyager 2 in 1981 [Bridge et al., 1982] and Cassini since 2004. The first three were merely flybys of the ringed planet as part of a much grander solar system tour. Cassini is the 
first and only orbiter to date providing a vast coverage from pole to pole across a timescale of seasons. The observations of Voyager 1 in the magnetosphere were accompanied by those of Voyager 2 as an upstream solar-wind monitor and this was the only time when multi-spacecraft observations were possible. The spatial coverage, however, was very restricted where Pioneer's and Voyager's trajectories spanned 0430 and 1300 Saturn Local Time (SLT). The latitudinal coverage was also limited to the dayside.

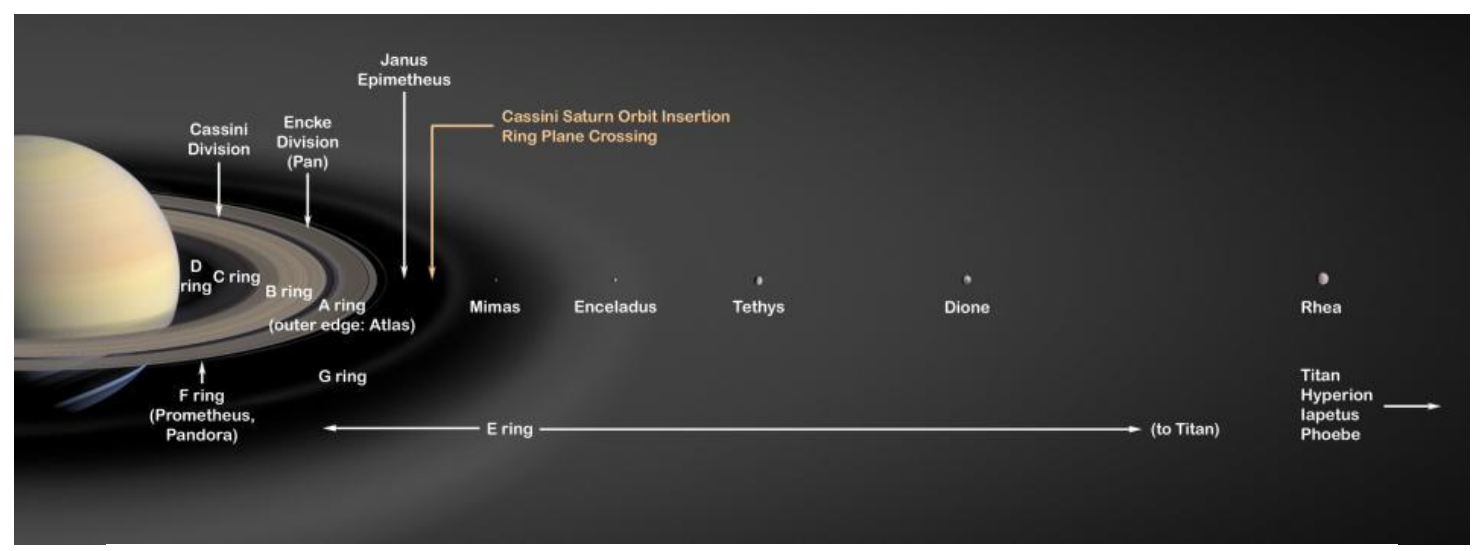

Figure 2.3.: Artist's impression of the Saturnian ring system depicting the extent of different ring segments and locations of the icy moons. [Credit: NASA]

\subsubsection{Saturn's Magnetosphere}

Saturn, like most planets in the solar system, generates its own intrinsic magnetic field. The influence of a planetary magnetic field extends far beyond the planet's immediate vicinity to form a magnetosphere. This is a largely impenetrable cavity that shields the planet from the incoming solar wind flow owing to their respective magnetic fields being "frozen in" to the plasmas. The size and shape of the magnetosphere is principally dictated by the balance of competing (external) solar wind and (internal) planetary magnetic field momentum fluxes. The boundary between these two regions is called the magnetopause, across which the magnetic field magnitude and direction changes abruptly thus forming a sheet of current at the interface commonly known as the Chapman-Ferraro current [Chapman and Ferraro, 1930].

Figure 2.4 highlights the main and important features of Saturn's magnetosphere. The magnetosphere adopts a bullet shape on the dayside of the planet that 


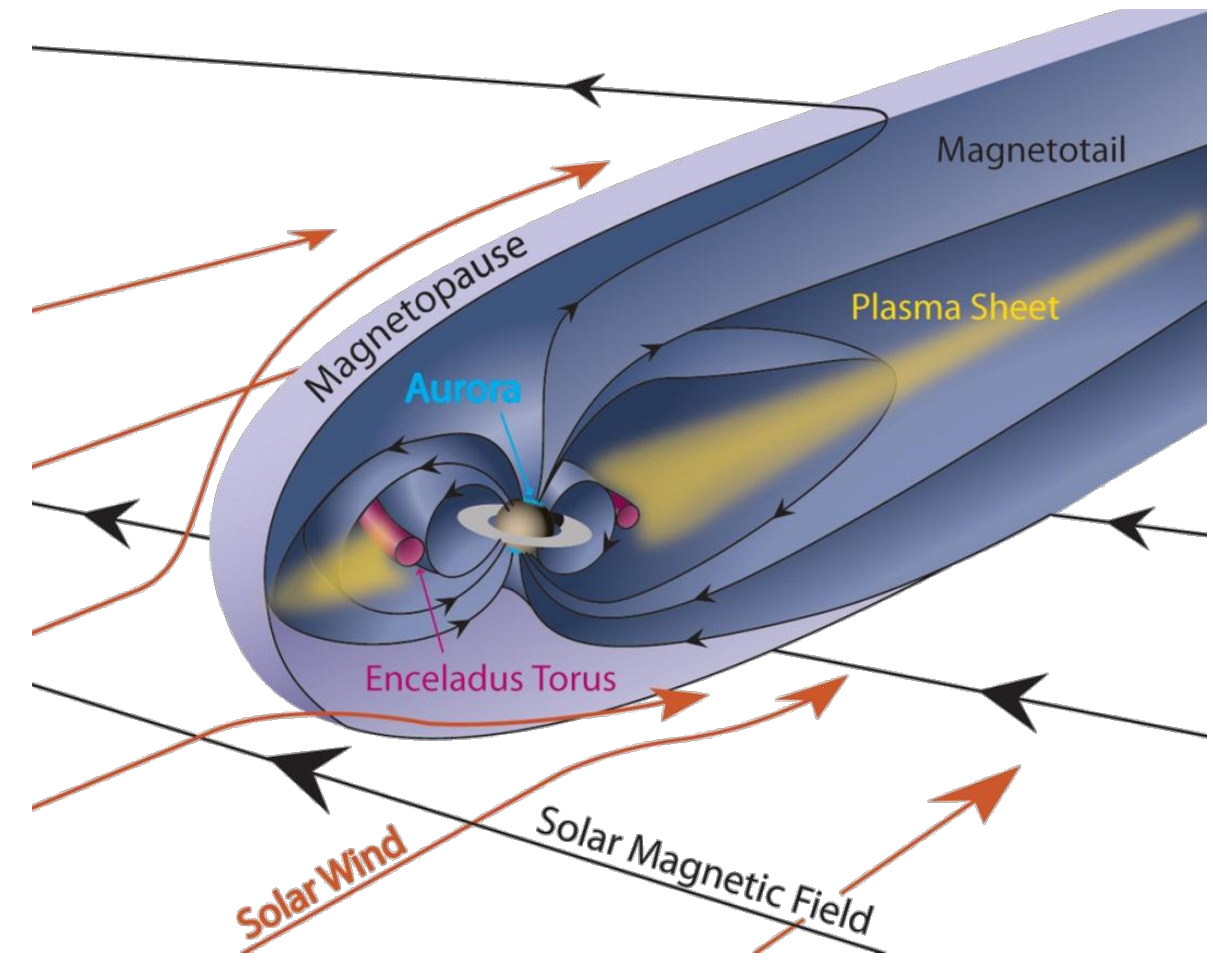

Figure 2.4.: Saturn's magnetosphere. [Credit: Fran Bagenal and Steve Bartlett]

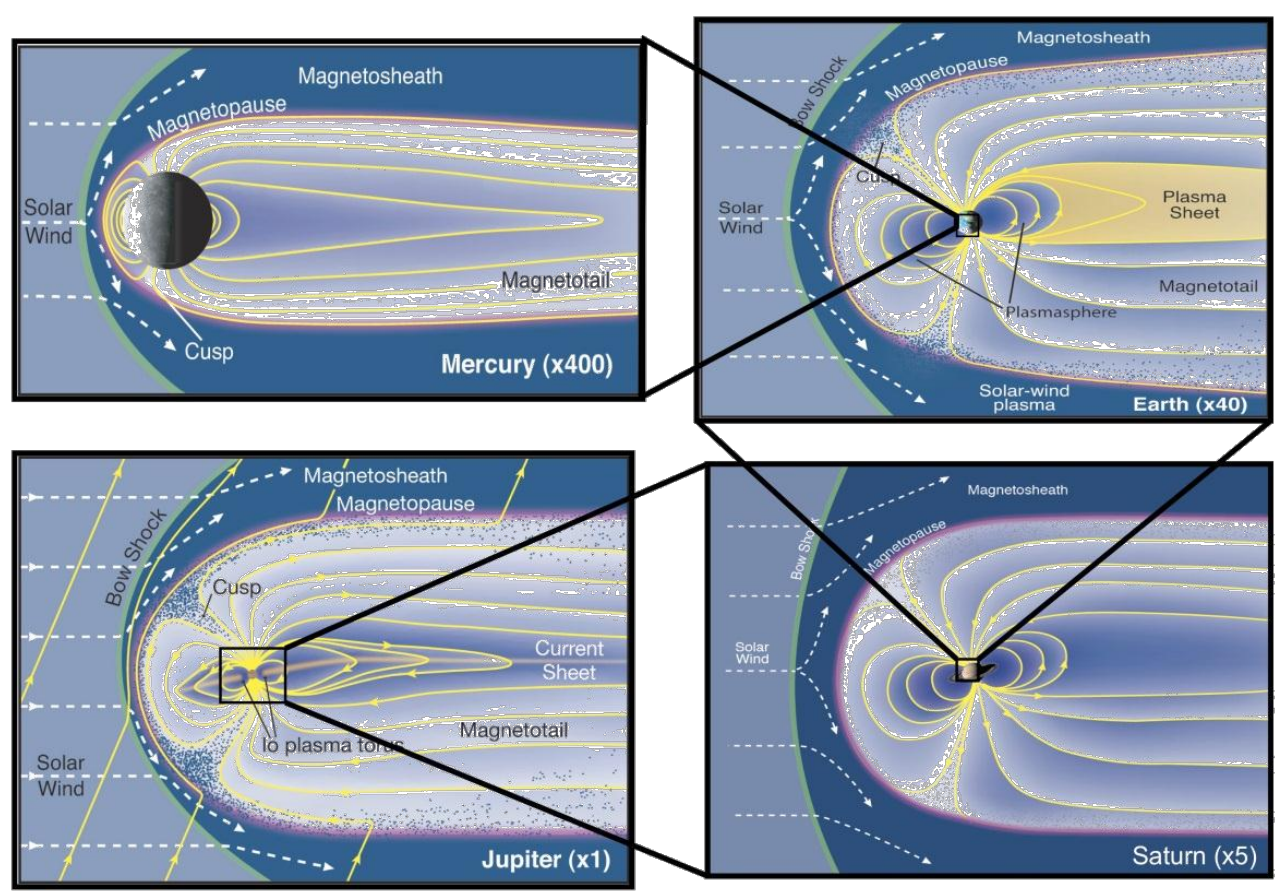

Figure 2.5.: Comparison of planetary magnetospheres: Mercury, Earth, Saturn, Jupiter. [Credit: Fran Bagenal and Steve Bartlett] 
is confined by the solar wind and on the nightside, it stretches far downstream into a long trailing structure called the magnetotail. A plasma sheet stretches down the length of the magnetotail at the magnetic equator i.e. between oppositely directed field lines [Jackman et al., 2007]. Recent studies using simultaneous Cassini and Hubble Space Telescope observations associate the production of Saturn's aurora to field-aligned currents [Bunce et al., 2008]. Arguably the most influential driver of Saturn's magnetosphere is the mass loading of plasma by Enceladus at $5 \mathrm{R}_{\mathrm{e}}$. This continuous outgassing leads to the formation of a waterdominated plasma torus around Enceladus's orbit. This leads to a RayleighTaylor instability setting up where the cold and dense inner plasma is interchanged with hot and tenuous from the outer magnetosphere. This process is driven by the outward centrifugal force associated with Saturn's rapid rotation rate [Thomsen et al., 2013]. The standoff distance (i.e. the planetocentric distance to the nose) of the magnetopause can be approximated by the balance of the solar wind dynamic pressure and the planet's magnetic pressure.

Figure 2.5 gives an idea of spatial dimensions and one can characterise the scales of the different planets' magnetospheres by determining how much the magnetic pressure can stand off the impinging plasma pressure above the surface. The principle is the same for all planets; both internal and external parameters controlling the structures with their respective uniqueness stemming from the relative contributions of these parameters. Mercury is the only planet with a surface magnetic pressure comparable to the ram pressure thus barely standing off the external plasma above its surface. At Saturn, there is a population of Enceladus-sourced plasma which appreciably contributes to the internal (outward) pressure, $P_{M}$ [Arridge et al., 2006; Kanani et al., 2010]. This position at the nose is thus approximates by

$$
\rho u^{2}=\frac{B^{2}}{2 \mu_{0}}+P_{M}
$$

The distribution of standoff distances was found to be probabilistically described by a bimodal model at 22 and $27 \mathrm{R}_{\mathrm{s}}$ [Achilleos et al., 2008]. This bimodality, however, was not apparent in the solar wind which suggested other mechanisms 
were influencing the size of the magnetosphere. This was attributed but not limited to the Vasyliunas cycle, a process set up by Saturn's rotation to expel internal plasma thus counterbalancing Enceladus' mass loading.

Embedded within Saturn's magnetosphere is a thin equatorial sheet of electrical current and energetic particles called the magnetodisc [Arridge et al., 2008]. Its geometry is controlled by the balance between the centrifugal force and mechanical stresses at a radial distance 20-30 $R_{S}$ from Saturn. The presence of the magnetodisc leads to a significant distortion in the magnetosphere which is inflated in the equatorial region and flattened at the poles.

\subsubsection{Dungey vs Vayliunas Cycles}

Under favourable upstream conditions, the "frozen-in" assumption of ideal magnetohydrodynamics breaks down and magnetic reconnection on the dayside magnetopause takes place. Here, the IMF and planetary magnetic fields merge resulting in magnetic flux tubes opening and solar wind plasma penetrating the magnetosphere. This is a means of the solar wind imparting momentum and energy into the planetary system. These open flux tubes, anchored to the planet at the other end, are then convected with the deflecting solar wind over the planetary poles and into the nightside. In the magnetotail, open flux tubes are brought closer together, owing to the work done by the solar wind in bending the magnetic field lines, to eventual reconnection thereby conserving the overall flux of the system. A portion of the magnetic energy is converted to mechanical energy, accelerating and heating the local plasma into planet- and anti-planetward jets.

The Dungey cycle is a dominant process in solar wind-driven magnetospheres such as the Earth and Mercury. By contrast, Saturn is a rotationallydriven magnetosphere and the Vasyliunas cycle becomes an important alternative mechanism for mass loss [Vasyliunas, 1983]. The continuous mass loading of Enceladus means that there must exist a combined loss mechanism at approximately the same rate to maintain global equilibrium, otherwise the magnetosphere would continue to inflate indefinitely. In the Vasyliunas cycle, the internal plasma is picked up by Saturn's magnetic field and accelerated to nearly corotational speed [Hill, 1979]. The centrifugal interchange instability transports the 
plasma outwards and exchanges the (radially inner) cold and dense for (outer) hot and tenuous plasma-filled flux tubes [Southwood and Kivelson, 1989]. As the flux tube rotates to the nightside, it is no longer confined by the magnetopause and continues to expand migrate tailward. This expansion stretches the magnetic field until oppositely-directed field lines become close enough to reconnect. This "pinches off" a closed loop of magnetic field called a plasmoid. The release of plasmoids (containing internal plasma) is thought to exhibit a clock-like repetition with a timescale comparable to Saturn's rotation rate [Rymer et al., 2013].

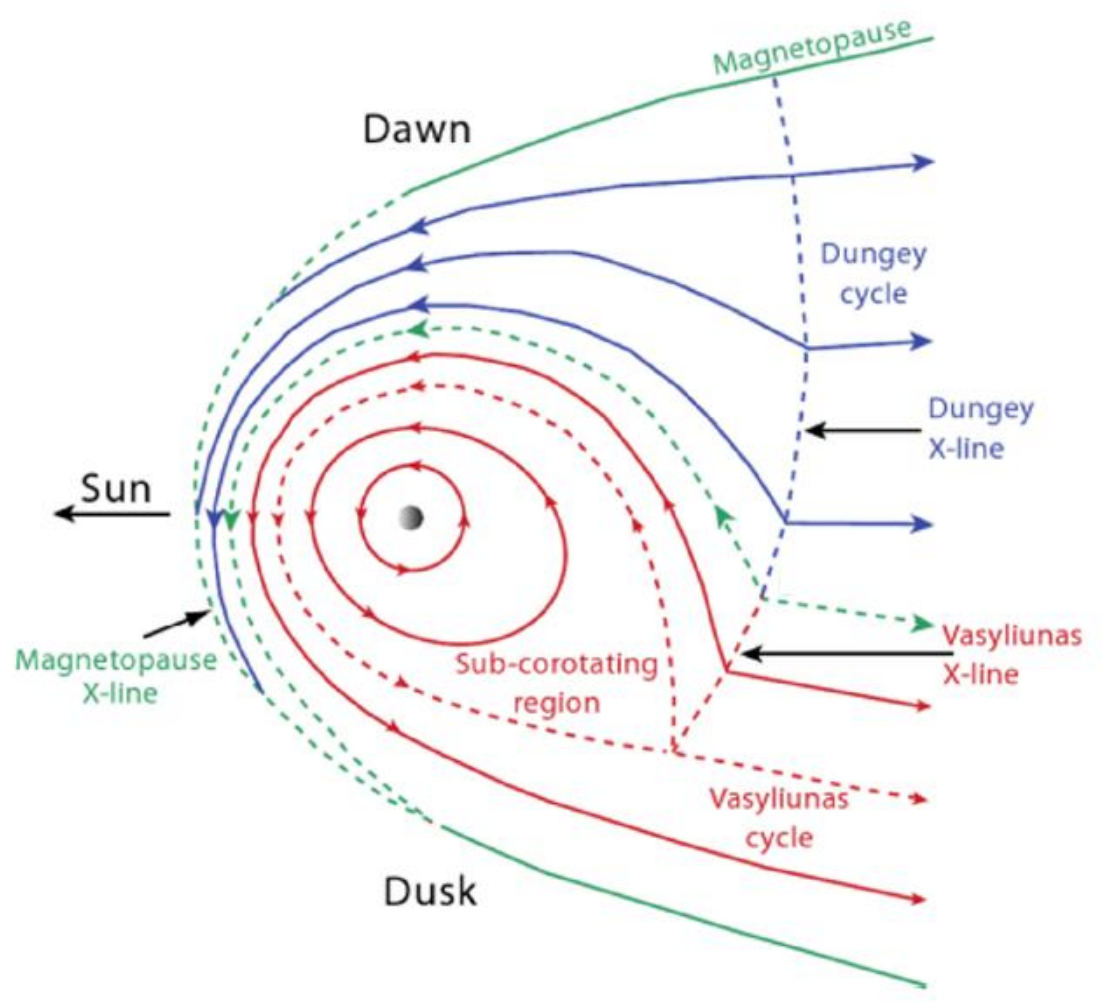

Figure 1.6.: Combined Dungey and Vasyliunas flow in the equatorial plane at Saturn [Cowley et al., 2004].

Figure 2.6 is a model combining the region dominated by the Vasyliunas cycle (red), i.e. the corotation of plasma, and the region dominated by the Dungey cycle (blue). Here, the rapid rotation and radial outflow associated with the Vasyliunas cycle leads to a deviation of the Dungey cycle reconnection X-line towards the dawn flank of the magnetopause. The hot and tenuous plasma from the reconnection along this X-line forms a sunward flow in the flank. This is called the Dungey cycle return flow and is typically associated with the azimuth- 
al flow in the outer magnetosphere in contrast with the inner (also sunward) flow from the Vasyliunas return cycle [Kivelson and Southwood, 2005; Badman and Cowley, 2007].

\subsubsection{The KSM Coordinate System}

The coordinate study used throughout this thesis is the Cartesian Kronocentric Solar Magnetic (KSM) system which centres Saturn at the origin, with positive $\widehat{\boldsymbol{X}}$ pointing towards the Sun, $\widehat{\boldsymbol{Y}}$ orthogonal to the magnetic dipole axis $\widehat{\boldsymbol{M}}$ (approximately aligned with the rotation axis at Saturn) and pointing towards dusk, i.e. $\widehat{\boldsymbol{M}} \times \widehat{\boldsymbol{X}}$, and $\widehat{\boldsymbol{Z}}$ chosen such that the magnetic dipole axis $\widehat{\boldsymbol{M}}$ is contained in the $\boldsymbol{X}-\boldsymbol{Z}$ plane with positive $\widehat{\boldsymbol{Z}}$ pointing in the northward sense. Figure 2.7 illustrates this pictorially.

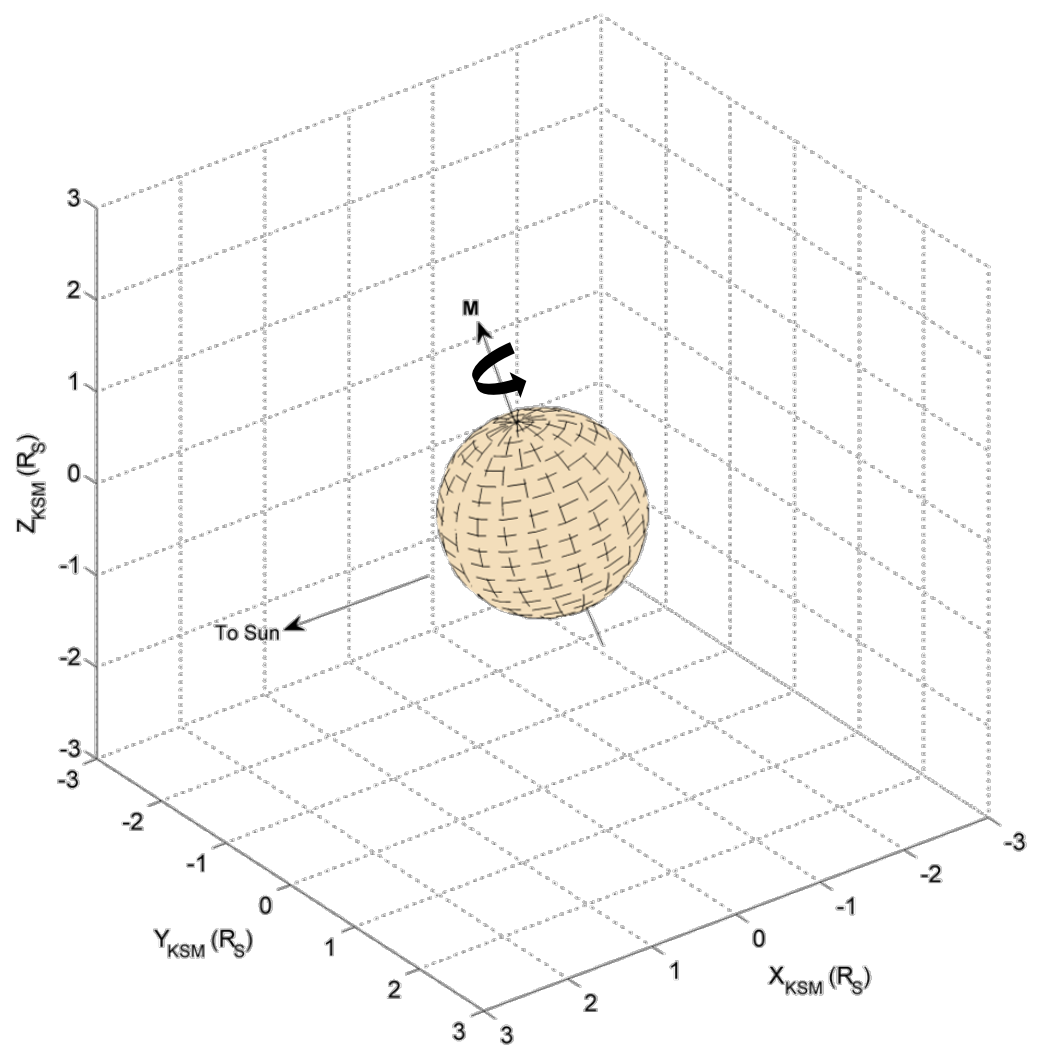

Figure 2.7.: Illustration of the KSM coordinate system as described in the in this subsection. 


\subsubsection{Cassini Observations of Saturn's Dayside Environment}

Figure 2.8 shows a magnetic field and spatial time history of Cassini travelling planetward. The spacecraft begins in the solar wind and encounters the quasi-perpendicular bow shock just before 2100. The region downstream, called the magnetosheath, is characterised by a sub-fast magnetosonic $\left(M_{f}<1\right)$ flow where solar wind is decelerated and deflected. The magnetic field strength is greater than that of the unshocked IMF; a requirement by the RH relations since the bow shock is a fast shock. The signature is also significantly noisier and these fluctuations are attributed to intrinsic turbulence in the solar wind with some generated within the shock itself [Skopke et al., 1983]. A common signature in the magnetosheath is a periodic anti-correlation between magnetic field strength and density fluctuations, which is indicative of so-called mirror mode waves [Violante et al., 1995]. These nonpropagating and compressive MHD waves are generated by the mirror instability which occurs in anisotropic plasmas when $T_{\perp} / T_{/ /}>1+1 / \beta_{\perp}$ [Southwood and Kivelson, 1993]. This condition is more easily met at Saturn's magnetosheath where $\beta$ is larger than at Earth. The anisotropy arises from the preferential heating across the shock by virtue of the conservation of the first adiabatic invariant i.e. the magnetic moment $\left(W_{\perp} / B\right.$; where $W$ is the particle's kinetic energy).

Across a wide range of timescales, foreshock waves, non-linear and/or transient structures can contribute to fluctuations sometimes being comparable to the background field. Based on measurements at the Earth, Sibeck and Gosling (1996) also interpret the origin of these fluctuations as radial gradient of the flow parameters with radial motion of the bow shock and magnetopause boundaries. Globally, the solar wind-embedded field lines in this region forms a draping pattern around the magnetopause. Figure 2.9 is taken from one of the earliest works on the Earth's magnetosheath [Spreiter et al., 1966] and illustrates this draping pattern using magnetodydrodynamics. The specific case here is of the magnetic field being perpendicular to the flow streamline - a realistic case in the nearSaturn space. As a consequence of Equation 1.23b, the field lines can be seen to locally refract away from the shock normal and are advected in unison with the magnetosheath flow. A detailed and more tailored introduction to Saturn's mag- 
netosheath can be found in $\S 6$. The magnetic field of Saturn points from north to south, i.e. in the opposite sense to Earth. Typically, a sharp rotation of the field to $B_{z}<0$ is observed at the magnetopause when Cassini is at the equator. This is not the case in Figure 2.8 which shows an atypical event when there is the field has a steady southward component throughout the traversal.
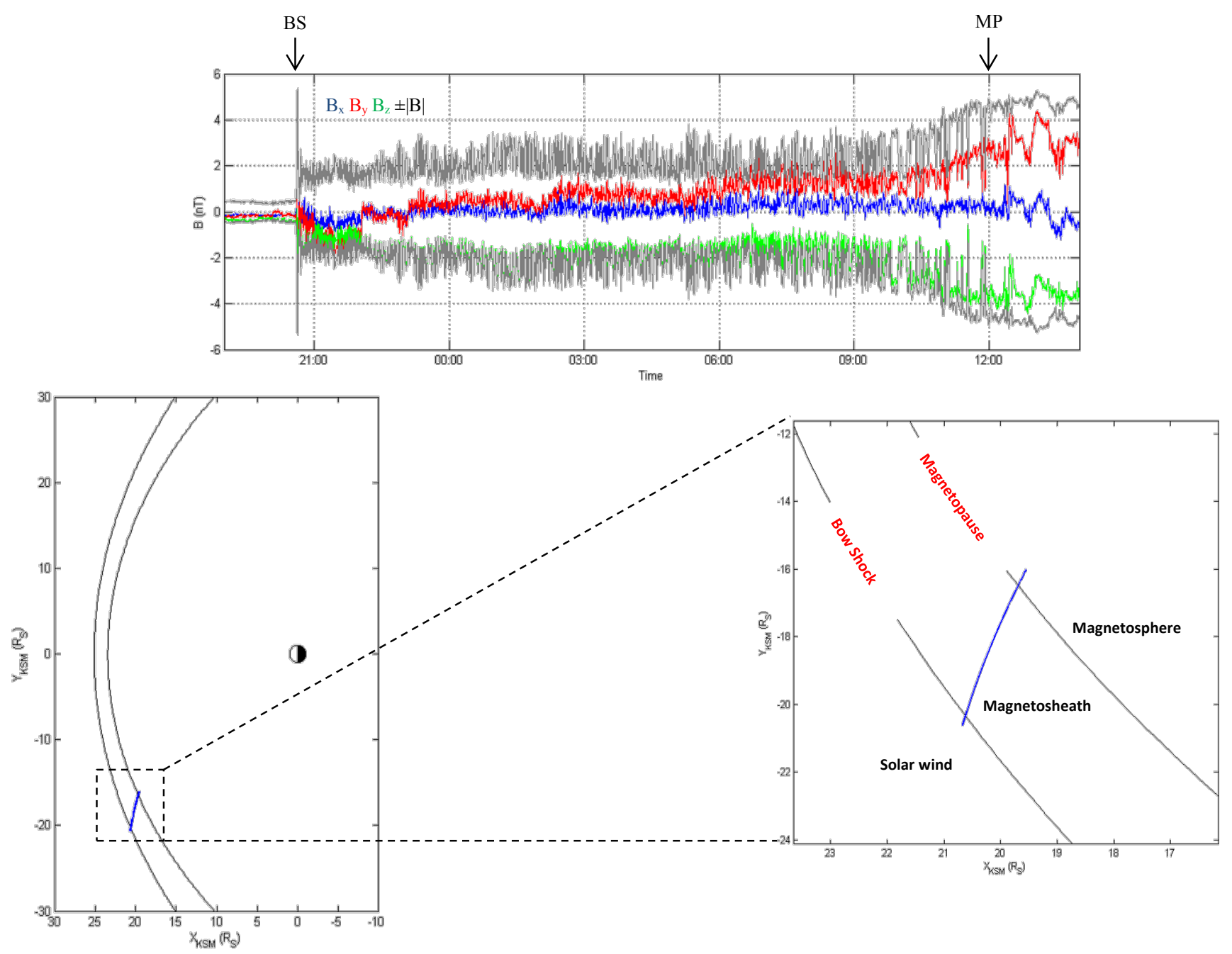

Figure 2.8.: Time series of magnetic field observation by Cassini on 29 and 30 July 2005 (top panel) and corresponding trajectory with respect to the bow shock (BS) [Went et al., 2011] and magnetopause (MP) [Kanani et al., 2010] boundaries (bottom panels). 


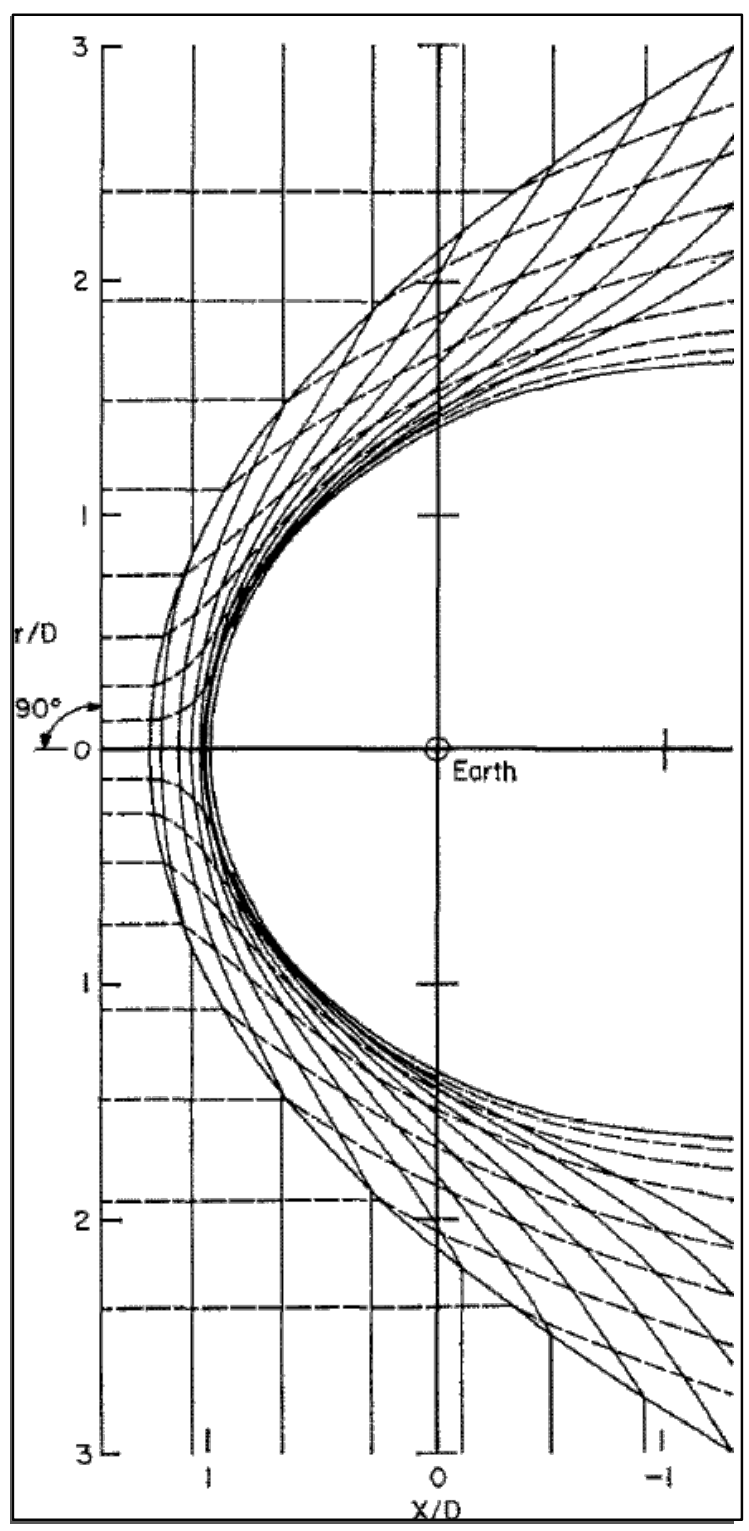

Figure 2.9: Global draping of the IMF around Earth's magnetosphere. The Sun is to the left of the horizontal axis connected to Earth. The first boundary (from the Sun) is the bow shock and second boundary the magnetopause. Parallel horizontal lines represent flow stream lines and parallel vertical lines represent the convected IMF [Spreiter et al., 1966]. 


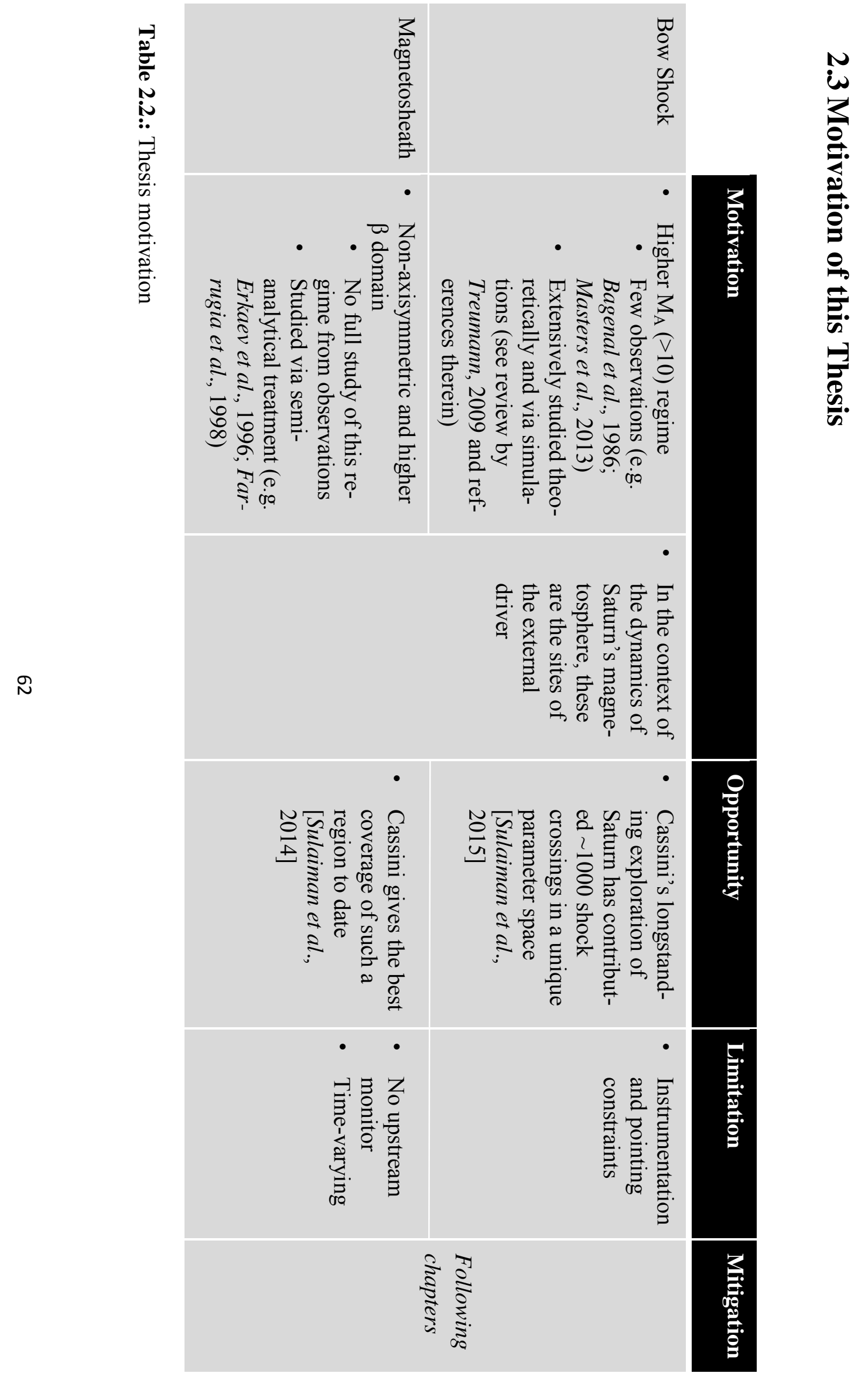




\subsubsection{Saturn as a Unique Laboratory for Collisionless Shocks}

The planets in the solar system are located at heliocentric distances which collectively cover a vast range of solar wind conditions. In situ spacecraft observations thus provide an insight into the solar wind properties and their interactions with, for example, planetary magnetospheres. This knowledge can also be extended to exoplanetary systems believed to be similar to those of the solar system.

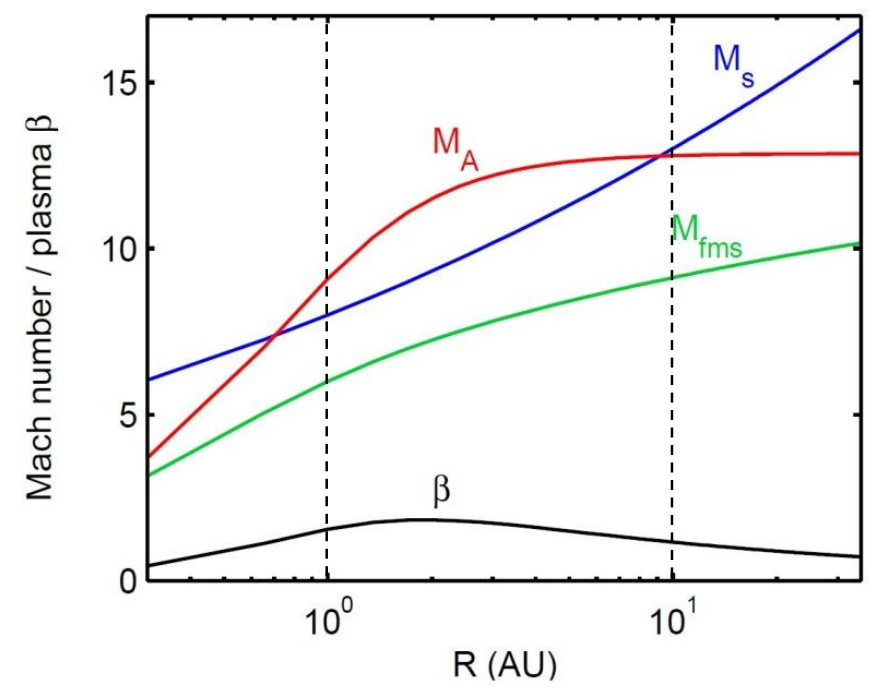

Figure 2.10.: Evolution of Mach numbers and plasma $\beta$ as a function of heliocentric distance. The dashed lines represent the locations of Earth at $1 \mathrm{AU}$ and Saturn at $10 \mathrm{AU}$.

The problem of collisionless shock wave dynamics, especially at high Mach numbers, is of wide interest not only to the solar system community but also to the astrophysicists. Supernova shocks, for example, are characterised by very high Mach numbers and their exploration is only limited to remote observations and simulations. At $10 \mathrm{AU}$, Saturn is in a unique position in the heliosphere where the Mach numbers are significantly higher than are available at Earth. Saturn's bow shock therefore represents an excellent laboratory for exploring the behaviour of such shocks. The opportunity at Saturn is already reflected in a recent publication by Masters et al. (2013) where relativistic electrons were discovered at Saturn's bow shock - a process expected to occur at supernova rem- 
nants. This work has already led to complementing recent simulations of such shocks [Guo and Giacalone, (2014)].

Figure 2.10 shows the typical solar wind dimensionless parameters as a function of heliocentric distance. When the radial profiles of each state variable is looked at individually from Figure 2.1 (i.e. solar wind velocity, number densities and temperatures of ions and electrons, magnetic field), it can be seen that the Mach number evolution is principally controlled by the wave speeds i.e. $\left|\Delta v_{f, A, s}(R)\right| \gg|\Delta u(R)|$. Here the fast magnetosonic speed is calculated for a propogation perpendicular to the magnetic field. The correction, to account for propogation direction, would be almost negligible with increasing heliocentric distance where the IMF becomes nearly perpendicular to the solar wind velocity. For all Mach numbers, there is a monotonic increase while the $\beta$ changes marginally.

\subsubsection{Scope and Limitations of this Thesis}

Until Cassini, observations of the highest Mach number shocks were made from spacecraft flybys. In this chapter, we exploit the long term presence of Cassini in a high Mach number regime of the solar system. We are therefore able to expand on the work by Achilleos et al. (2006) which presented a set of several crossings from SOI. This work will characterise Saturn's bow shock using the largest sample of crossings to date and improved techniques from previous works. Statistical studies of Earth's bow shock have been made [e.g. Henley, 2010] for modest Mach numbers in the range $M_{A}=2-8$. Here we have a much larger range of Mach numbers spanning two orders of magnitude. A subset of these crossings will be studied and discussed in Chapter 5 where Saturn's bow shock will be discussed as a prototype of high Mach number shocks in the astrophysical-like regime.

Cassini is of course a single spacecraft and this comes with its associated limitations. One is the capability to separate spatial and temporal variability in the observations. Direct inference of the shock speed, for example, cannot be made reliably. Multi-spacecraft timings are used at Earth to measure the normal velocity of the bow shock as it propagates between two or more spacecraft. The 
next chapter introduces a well-established single-spacecraft technique to estimate this parameter. Another limitation is the operability of the plasma instruments. Measurements of the solar wind speed require the instrument's field-of-view to be in the direction of the incoming flow are outlined in Chapter 3. 


\section{Spacecraft and Instrumentation}

The new scientific work in this thesis stems from data gathered and returned to Earth by the Cassini-Huygens spacecraft. Onboard the spacecraft is a suite of in situ instruments making field, particle and wave measurements for studying dust, plasma and magnetic fields around Saturn. There are also suites of optical remote sensing instruments (e.g. UV and infrared imaging) for studying Saturn's aurora and microwave remote sensing instruments (e.g. radar) for mapping Saturn's atmosphere and its moons' surfaces. Figure 3.1 shows where each of the twelve instruments are located on the spacecraft. The data presented and interpreted in these studies are principally from Cassini's Fluxgate Magnetometer (MAG) [Dougherty et al., 2004] with supporting data from the Radio and Plasma Wave Science (RPWS) [Gurnett et al., 2004] and Ion Mass Spectrometer (IMS) [Young et al., 2004]. In this chapter, we will begin with a brief overview of the mission and then describe the instruments used.

\subsection{The Cassini-Huygens Spacecraft}

In situ measurements of the Saturnian environment were first made by spacecraft flybys Pioneer 11 (1979), Voyagers 1 (1980) and 2 (1981) which led to a significant leap in our understanding of gas giant systems [Acuna and Ness, 1980; Smith et al., 1982; Ness et al., 1981] as well as posing new questions, most notably the source of the planet's 10.7 hour period oscillations [Espinosa and Dougherty, 2000].

Launched in October 1997, Cassini embarked on a seven-year journey to Saturn, marking the beginning of an unrivalled exploration of the gas giant. The joint American and European enterprise would be the first Saturn orbiter to provide the largest spatial and temporal coverage of that planetary system to date. The Huygens probe was deployed onto Saturn's largest moon, Titan, a few months after Saturn Orbital Insertion (SOI). 


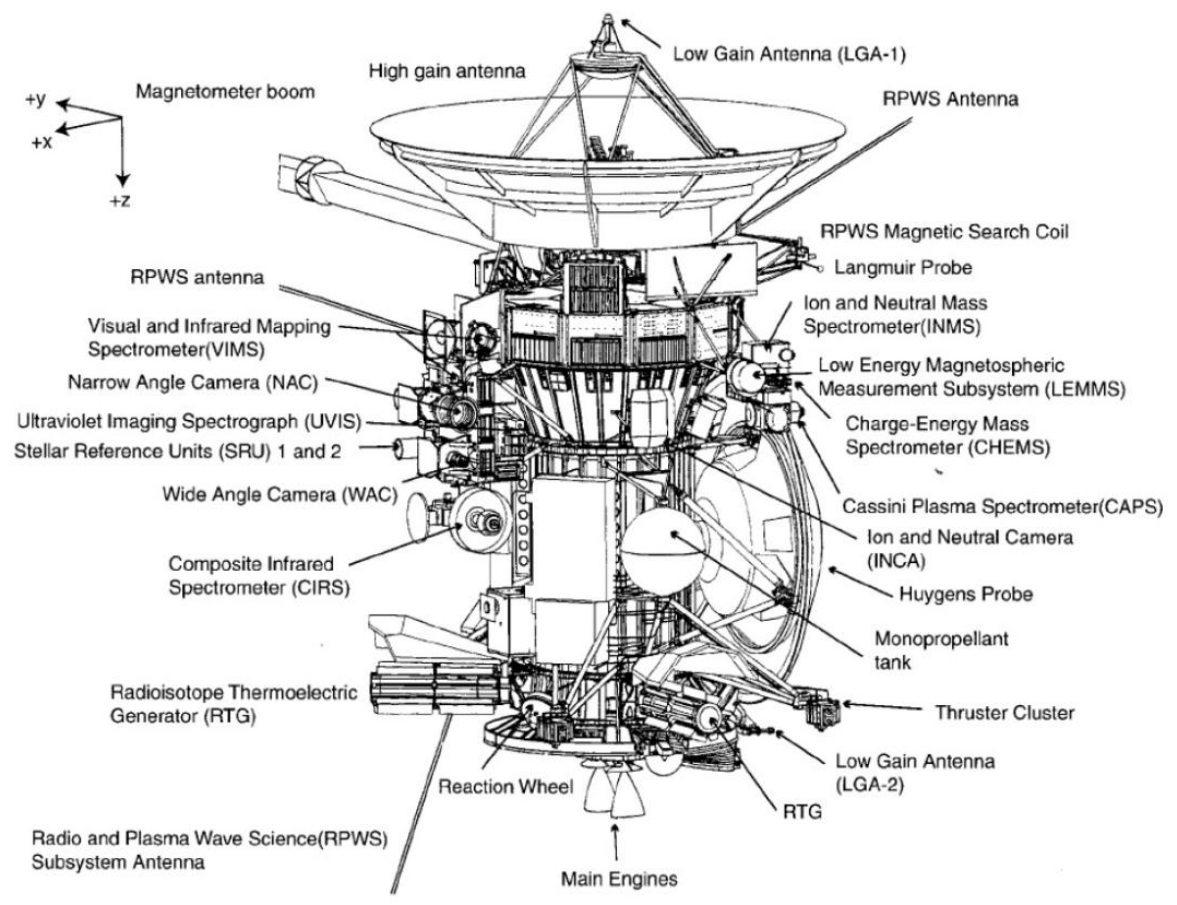

Figure 3.1.: Cassini spacecraft showing science instruments and some of the engineering subsystems. The magnetometer boom is aligned with the $+y$ axis and the high-gain antenna is pointed towards $-z$ [Burton et al., 2001].

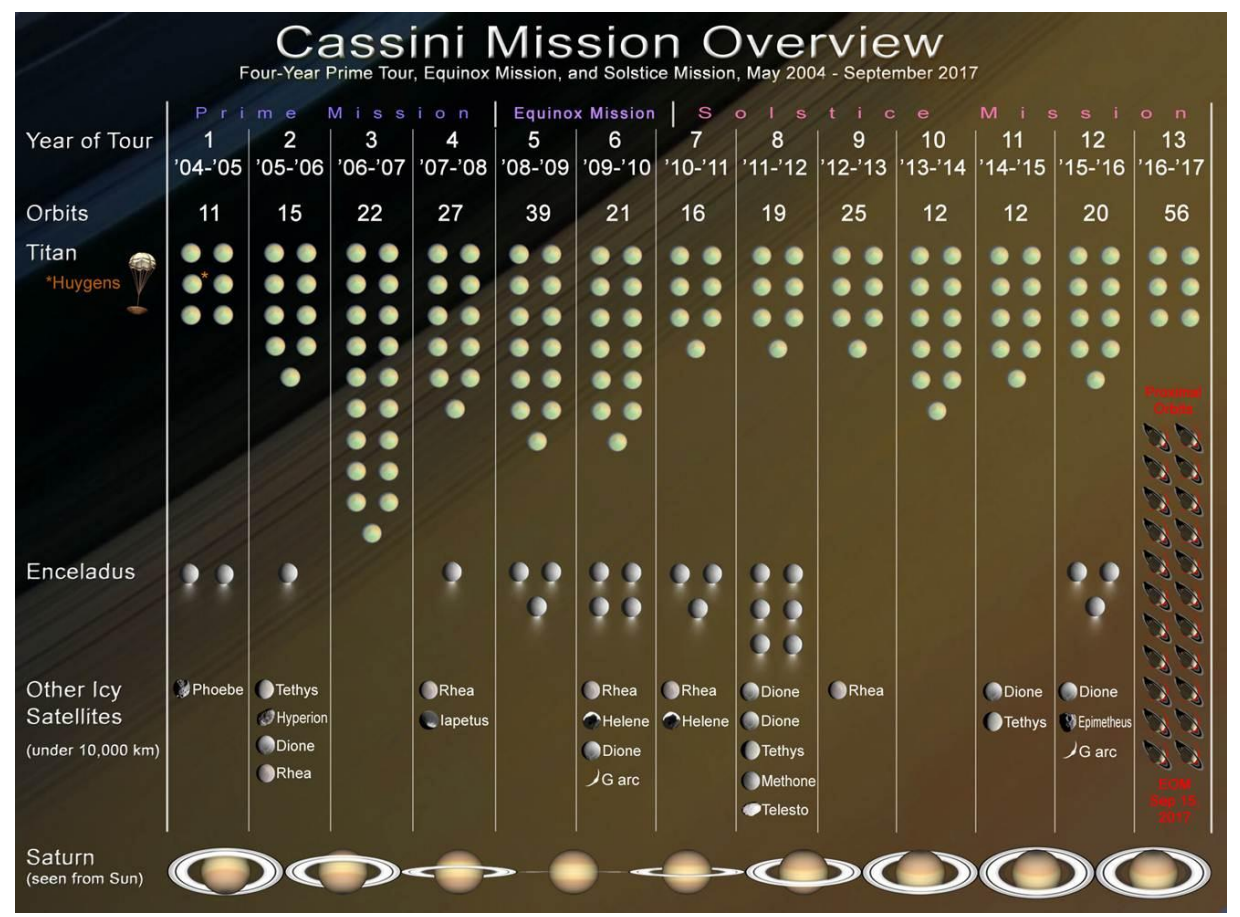

Figure 3.2.: Mission timeline of Cassini from Saturn Orbit Insertion (SOI) in 2004 until the planned proximal orbits in 2017. Satellite fly-bys and seasons are indicated [Credit: Dr Linda Spilker, JPL]. 
Cassini is powered by three radioisotope thermoelectric generators (RTGs) and relies on thrusters and a set of reaction wheels to propel, retard and orient itself. The reaction wheels are mounted orthogonally at three axes and provide the required spacecraft attitude via angular momentum exchange. The spacecraft is three-axis stabilised which introduces constraints on full particle coverage as well as complications in calibrating some instruments. The magnetometer, for instance, relies on spacecraft rolls where the ambient magnetic field is relatively steady to mitigate systematic offsets in the data (more on this in the next subsection).

The accomplished and planned mission highlights are summarised in Figure 3.2. The most visited satellites are the atmospherically-rich Titan [Porco et al., 2005] and the geologically-active Enceladus [Dougherty et al., 2005]. In addition to the prime mission which lasted 4 years after SOI, the mission has been extended twice through equinox and solstice and is now due to end in September 2017.

\subsection{Cassini Fluxgate Magnetometer}

The magnetometer is one of the most crucial instruments on a space mission. In situ measurements of the magnetic field offer the scope to address a vast range of plasma physics problems. Since the structures and dynamics of space plasmas and their constituents are controlled by the Lorentz force, knowledge of the magnetic field is required. This spans from small length scales where particle gyration is important to much larger length scales where collective fluid behaviour dominates (i.e. $k \lambda_{D} \ll 1$ ). In the solar wind, observations of the IMF provide information about the development and evolution of a variety of processes. On large scales, multi-point measurements of the magnetic field across the heliosphere reveal the expansion of the solar wind as it travels away from the Sun; including transient events such as Coronal Mass Ejections (CMEs) [Klein and Burlaga, 1982]. On smaller scales, fluctuations in the magnetic field data can be used to characterise the turbulence inherent in the flow. Here the energy cascades from large-scale structures through a hierarchy of smaller and smaller eddies until viscous dissipation takes place. In an energy spectrum with increasing wave 
number, $k$, these fluctuations in the data are manifested as a power law with a distinct (and mostly more than one) spectral index depending on the range of scales [Goldstein et al., 1995].

Figure 3.3 shows a photograph of Cassini's Fluxgate Magnetometer (FGM) [Dougherty et al., 2004]. This is one of the spacecraft's two magnetometers; the other being the Scalar/Vector Helium Magnetometer (S/VHM). The design of the S/VHM shall not be discussed in detail here since its data is not used. Both magnetometers are mounted on the spacecraft's 11-meter boom at the halfway and end points (increasing $y_{s / c}$ ) respectively. This distance from the spacecraft keeps the interference of the magnetic fields induced by the spacecraft's electronics at an acceptable minimum of $\sim 0.6 \mathrm{nT}$ [Kellock et al., 1996].

Flying with two magnetometers has two advantages: one being redundancy - which has already proved indispensable since the S/VHM ceased to operate and the other being in-flight calibration. Calibration becomes necessary when the time-varying drift of the zero level becomes substantial. This is still achieved in spite of S/VHM's absence through spacecraft rolls, which have been mostly carried out at a period of approximately one hour in the quiet fields. Cassini is capable of rolling faster with the aid of its thrusters during mission segments when the spacecraft spends most of its time in the inner magnetosphere (e.g. in the upcoming proximal orbits).

Cassini's FGM is very similar to the version flown onboard the Ulysses spacecraft [Balogh et al., 1992] and both were built at Imperial College. This magnetometer is made up of three sensors in a triaxial setup, with each measuring a component of the ambient magnetic field. A sensor comprises a highly magnetically permeable ring core of two separate halves around which are wrapped two coil windings: a drive (primary) winding and a sense (secondary) winding, as illustrated in Figure 3.4. The drive winding is wrapped around the ring core toroidally while the sense winding is wrapped around a plane containing the ring core and drive winding. 


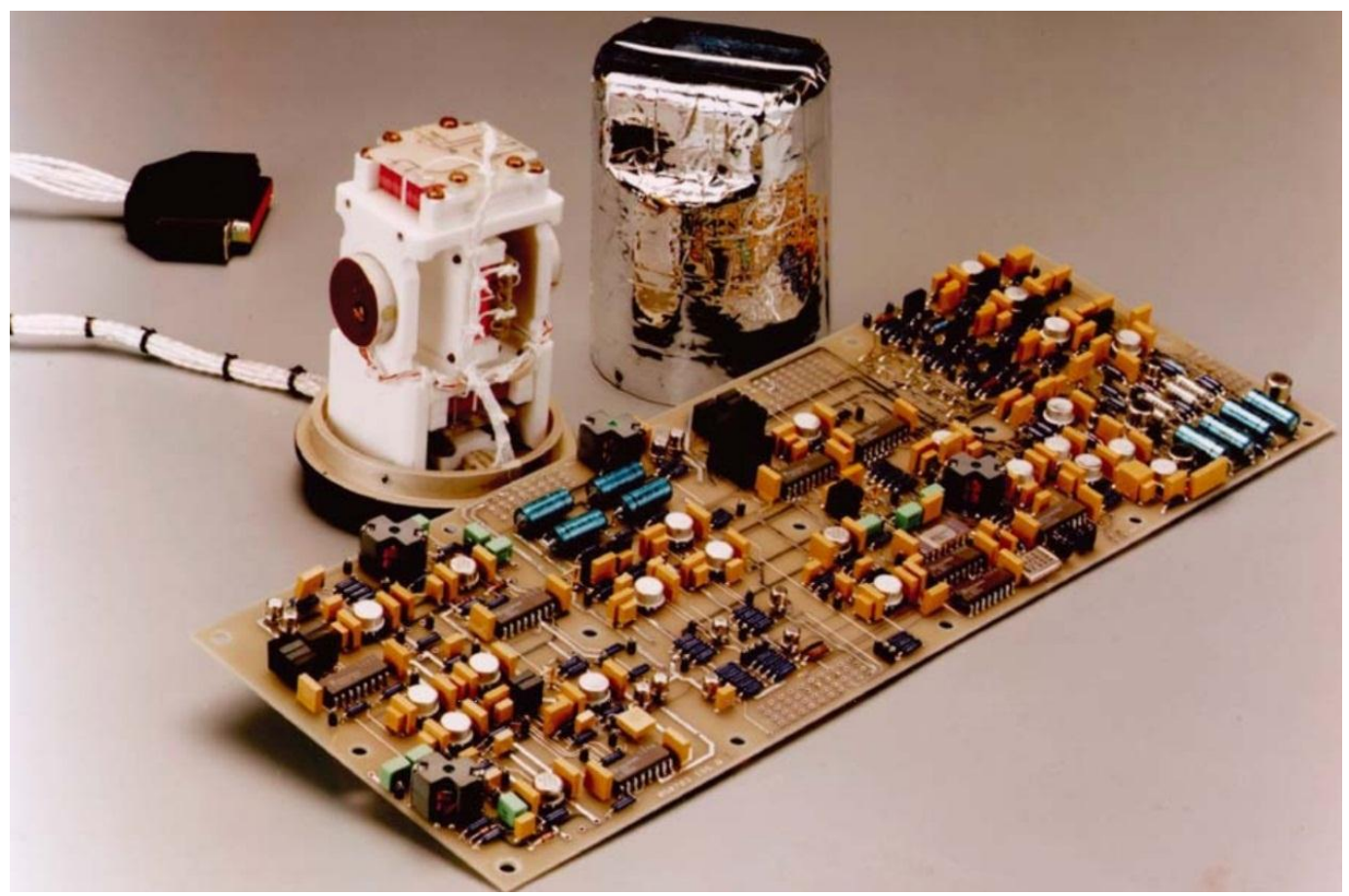

Figure 3.3.: Photograph of the FGM and electronics board with cover off.
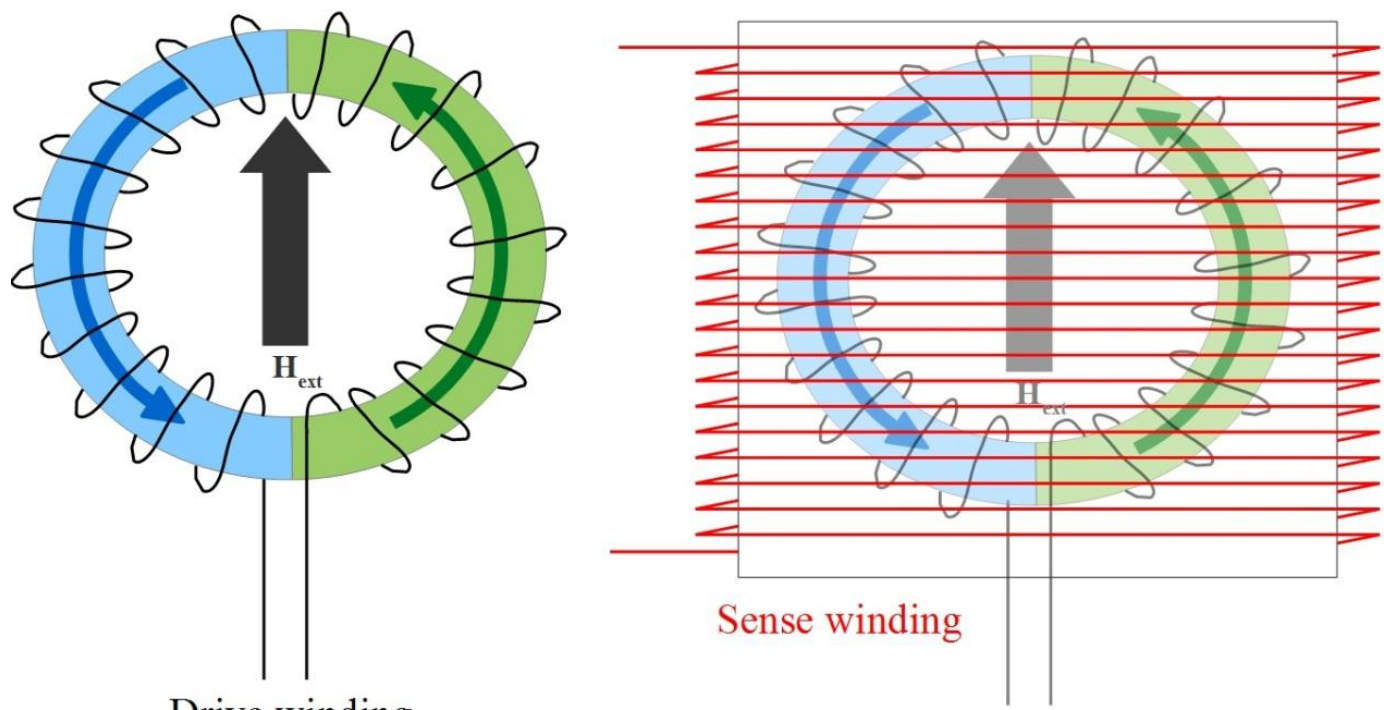

Drive winding

Figure 3.4.: Schematic of ring core setup to measure the external magnetic field in the direction of $H_{e x t}$ with the drive winding (left figure) and sense winding (right figure) configurations. [Courtesy of Imperial College and redrawn for improved image quality.] 
The arrangement in Figure 3.4 measures the field in the direction of $H_{\text {ext }}$. As an alternating current passes through the drive winding, one half core will generate a field in the same sense (green arrow) as $H_{e x t}$ while the other generates a field in the opposite sense (blue arrow). This current is in the form of a square wave at a frequency of $f_{0}=15.625 \mathrm{kHz}$ and magnetises the core to saturation twice per cycle in the $M-H$ hysteresis loop. Both half cores go through the same $M-H$ loop and are $180^{\circ}$ out of phase from each other. From Faraday's law, any time-varying magnetic field will be picked up by the sense winding as an induced secondary voltage. In the absence of an ambient field $\left(H_{e x t}=0\right.$, and assuming there is no offset), both halves simultaneously go into and out of saturation. As a result, there is no net change of flux and thus no voltage is induced in the sense winding. When an ambient field is present, the half core generating a field in the opposite sense as $H_{\text {ext }}$ comes out of saturation sooner than the other half core. The situation is reversed in the second half of the cycle. Here the fields do no cancel out and the time-varying net flux now induces a voltage in the sense winding with the size being proportional to the magnitude of $H_{\text {ext }}$.

The Cassini FGM operates in feedback loop. The induced voltage in the sense winding is integrated and fed back into the sense winding to null the ambient field and the linearity of the instrument is improved this way. The instrument is capable of measuring the magnitude and direction of the ambient magnetic field up to 32 times a second. Table 3.1 lists the characteristics of the instrument. The combined noise performance of the instrument is better than $5 \mathrm{pt} / \sqrt{\mathrm{Hz}}$ at 1 $\mathrm{Hz}$.

\begin{tabular}{lllll}
\hline Mass & Sampling rate & Range & Dynamic range & Resolution \\
\hline $0.44 \mathrm{~kg}$ & 32 vectors/s & 0 & $\pm 40 \mathrm{nT}$ & $4.9 \mathrm{pT}$ \\
& & 1 & $\pm 400 \mathrm{nT}$ & $48.8 \mathrm{pT}$ \\
& & 2 & $\pm 10,000 \mathrm{nT}$ & $1.2 \mathrm{nT}$ \\
& & 3 & $\pm 44,000 \mathrm{nT}$ & $5.4 \mathrm{nT}$ \\
\hline
\end{tabular}

Table 3.1.: FGM Instrument Characteristics taken from Dougherty et al. (2004). 


\subsection{Calibration}

As aforementioned, the outputs from the FGM sensors incur offsets from the zero level thereby systematically deviating the measured magnetic field from the actual value. The offsets are not constant and drift with time which further complicates matters. An additional difficult situation arises from nonorthogonality of the boom. These complications are periodically mitigated and most straightforwardly achieved with a spinning spacecraft. A sensor arranged to measure the ambient magnetic field in a direction perpendicular to the spacecraft's spin axis should, in principle, measure a field of zero over one revolution. Any offsets will be picked up as a non-zero reading. This technique therefore calibrates the two sensors in the perpendicular arrangement. As for the third sensor, parallel to the spin axis, calibration depends on the position of the spacecraft. Typically there are two regions where calibration can most readily be conducted. The first is the solar wind where the magnitude of the field is more or less steady and the fluctuations attributed to changes in field direction. Here, identification of the offsets can be achieved statistically [Belcher, 1973]. The other is in the inner magnetosphere where the timescales at which the magnetic field varies are greater than the period of the spacecraft's spin. Changes in the magnetic field here are therefore attributed to the changes in the spacecraft orientation.

Calibrating the FGM in Cassini, a three-axis stabilised spacecraft, is a much more delicate task. It therefore relies on commanded rolls which are meticulously predetermined so as not to have an adverse impact on the other instruments. Factors taken into account include their field-of-view and temperature sensitivity from exposure to Saturn's radiated heat.

In addition to the techniques mentioned above, calibration for offsets and non-orthogonality can also be achieved from knowledge of the topology of magnetic structures, though this technique has not been used with FGM. These include internal field and/or magnetodisc current sheet models. In the latter, for example, it is known that during a current sheet crossing, the radial and azimuthal field components are zero. The other techniques described above remain essen- 
tial to the FGM's calibration programme from Earth's fly-by [Dunlop et al., 1999] through to the proximal orbits at the end of the mission.

\subsection{Cassini Radio and Plasma Wave Science (RPWS) Instrument}

The multi-purpose RPWS instrument comprises a suite of three orthogonal search coil magnetic antennas and three orthogonal electric field antennas to detect magnetic fields in a frequency range of $1 \mathrm{~Hz}$ to $12 \mathrm{kHz}$ and electric fields in a range of $1 \mathrm{~Hz}$ to $16 \mathrm{MHz}$ respectively [Gurnett et al., 2004]. A Langmuir probe is used to measure local electron density and temperature. The capability of RPWS also extends to detection of micro-dust particles.

Here we only use outputs from the Wideband Receiver which measures waves in the electric field using the three $10 \mathrm{~m}$ electric field antennas. A component of the electric field is proportional to the potential difference between a pair of antennas in the same plane. Every $125 \mathrm{~ms}$, a frequency-time spectrogram is then generated at a resolution of $13.6 \mathrm{~Hz}$ over a spectral range of $60 \mathrm{~Hz}$ to 10.5 $\mathrm{kHz}$. The parameter used in this work is the electron density, $n_{e}$ in $\mathrm{cm}^{-3}$, derived from the measured electron plasma frequency, $f_{p}=8980 \sqrt{ } n_{e} \mathrm{~Hz}$.

\subsection{Cassini Ion Mass Spectrometer (CAPS/IMS)}

CAPS/IMS is designed to measure velocity distributions of low-energy ion species up to $50 \mathrm{keV}$ [Young et al., 2004]. Ions travelling into the entrance aperture are accelerated into a detection chamber by an electric field set up by an applied voltage.

The instrument comprises eight anodes in a fan arrangement with an angular resolution of $8.3^{\circ} \times 20^{\circ}$ over a field-of-view of $8.3^{\circ} \times 160^{\circ}$. The sensor is capable of actuating to cover $2 \pi$ sr of the sky in $\sim 3$ minutes. A 64 -level logarithmic energy spectrum is then generated over a range of $1 \mathrm{eV} / \mathrm{q}$ to $50.3 \mathrm{keV} / \mathrm{q}$. The bulk flow velocity can be determined using CAPS/IMS when the instrument is looking into the incoming solar wind flow of sufficiently high density. Determination 
of the bulk flow speed is therefore restricted to desirable pointing. This instrument has also ceased to operate. 


\section{Magnetic Field Characterisation of Saturn's Bow Shock}

\subsection{Introduction}

\subsubsection{Size and Shape}

The magnetosphere of Saturn, like those of all magnetised planets in the solar system, is to first order an impenetrable blunt obstacle to the supermagnetosonic solar wind flow. In the context of solar system bodies, magnetospheres are not the only structures that present an obstacle to the incoming flow. Ionospheres can also lead to the formation of a fast magnetosonic shock wave and this has been notably observed at Venus [Russell et al., 1979] and more recently at Titan [Bertucci et al., 2015], for example.

At distances much greater than the planetary radius $\left(d \gg R_{p}\right)$, the shape of a shock wave asymptotes to that of a cone formed by the locus of wave fronts with the centre of the planet as the focus point [Landau and Lifshitz, 1959]. The angle of this 'Mach Cone', $\mu$, defined as the angle between the edge of the cone and the Sun-planet line, is dependent on the upstream Mach number, $M$, and given by

$$
\sin \mu=\frac{1}{M} ; M>1
$$

This equation is associated with the sonic Mach number, $M_{s}$, in gas dynamic theory [Spreiter et al., 1966]. In space applications, it has been found to be largely consistent with the fast magnetosonic Mach number, $M_{f}$, when fitted with observations of distant shock crossings of Venus, Earth and Mars [Slavin et al., 
1984]. With increasing heliocentric distance, the Mach cones of each planet were shown to become better in agreement with gas dynamic theory. This is attributed to the decrease in the IMF strength since

$$
M_{f}^{2}=\frac{M_{S}^{2} M_{A}^{2}}{M_{S}^{2}+M_{A}^{2}}
$$

by extension of Equation 1.21. In the limiting case of the IMF strength (and correspondingly $v_{A}$ ) decreasing, we have $M_{f} \stackrel{B \rightarrow 0}{\longrightarrow} M_{S}$.

At distances comparable to the planetary radius $\left(R_{p}<d \lesssim O\left(10^{2}\right)\right)$, a shock wave takes a size and shape similar to the obstacle [Billig, 1967]. Saturn's magnetosphere is a blunt body (as opposed to a pointed wedge) and a detached bow shock is thus formed in the dayside region [Slavin et al., 1985]. Cassini's orbits typically restrict bow shock crossings to the dayside region enabling detailed modelling of its three-dimensional size and shape [Masters et al., 2008; Went et al., 2011; the latter herein referred to as W11].

\subsubsection{Shock Frames of Reference}

The theoretical framework for the cross-shock potential is widely described in one of two commonly used frames of references, called the deHoffmannTeller (HT) frame. Here, the shock is at rest and the upstream flow vector is parallel to the upstream magnetic field [de Hoffmann and Teller, 1950]. The convenience of this frame is that the motional electric field vanishes as a result, thus equating the potential to the electron temperature gradient term in the generalised Ohm's law. In addition, from Equation 1.23f, the downstream flow and magnetic field vectors will also be parallel. The drawback to this frame is that it breaks down for perfectly perpendicular shocks since a shock requires a normal flow component [Schwartz et al., 1983].

The other is the Normal Incidence (NI) frame where the upstream flow vector is parallel to the shock normal. Unlike the HF frame, $\theta_{B n}$ is not preserved 
across the shock since the combination of jump in the magnetic field and conservation of $B_{n}$ (Equation 1.23b) results in a rotation away from the normal. This will be the preferred frame in this thesis because direct comparisons are made with simulations and earlier observations where Mach numbers are determined using only the normal flow component. The HF frame would be more convenient in studies looking at detailed particle distributions (e.g. the relationship between the cross-shock potential and electron heating).

\subsubsection{Determination of the Shock Normal}

Unlike an oblique shock wave, the bow shock's curvature introduces the complexity of global non-uniformity along its surface. In other words, each crossing must be associated with a unique normal vector $\widehat{\boldsymbol{n}}$ to the shock's surface at that point. Thus for a given upstream flow vector and IMF direction, the bow shock exhibits a range of $\theta_{V n}$ and $\theta_{B n}$ at any given time. This is in a sense analogous to an assembly of many "fragments" of planar shocks distributed across the surface, each with its specific set of upstream parameters and therefore processing the flow accordingly. The approximation of a planetary bow shock as thin and locally planar is valid since the radius of curvature is much larger than the shock width.

Two common techniques for determining the shock normal are described in this subsection with one of them used for reasons to follow. These techniques employ the Coplanarity Theorem and Model Boundary Equations. Both are used in single spacecraft analyses and therefore give rise to associated limitations.

The Coplanarity Theorem requires the local magnetic field vectors upstream and downstream to lie in the same plane as the local shock normal (see Figure 4.1). One form in which this can be expressed is given in equation 4.3 which only requires magnetic field data of a single crossing thereby making this technique the most straightforward. Other forms of this include the velocity vectors upstream and downstream of the shock, since the velocity jump across the shock lies in the same plane. This technique can be used in any frame [AbrahamShrauner, 1972]. 


$$
\widehat{\boldsymbol{n}}_{\boldsymbol{C}}= \pm \frac{\left(\boldsymbol{B}_{\boldsymbol{d}} \times \boldsymbol{B}_{u}\right) \times(\Delta B)}{\left|\left(\boldsymbol{B}_{\boldsymbol{d}} \times \boldsymbol{B}_{\boldsymbol{u}}\right) \times(\Delta B)\right|}
$$

where $\boldsymbol{B}_{\boldsymbol{u}}$ and $\boldsymbol{B}_{\boldsymbol{d}}$ are the magnetic field vectors upstream and downstream, respectively. The magnetic field jump across the shock is denoted as $\boldsymbol{A} \boldsymbol{B}\left(\equiv \boldsymbol{B}_{d}-\boldsymbol{B}_{\boldsymbol{u}}\right)$. The unit normal direction conventionally points upstream.

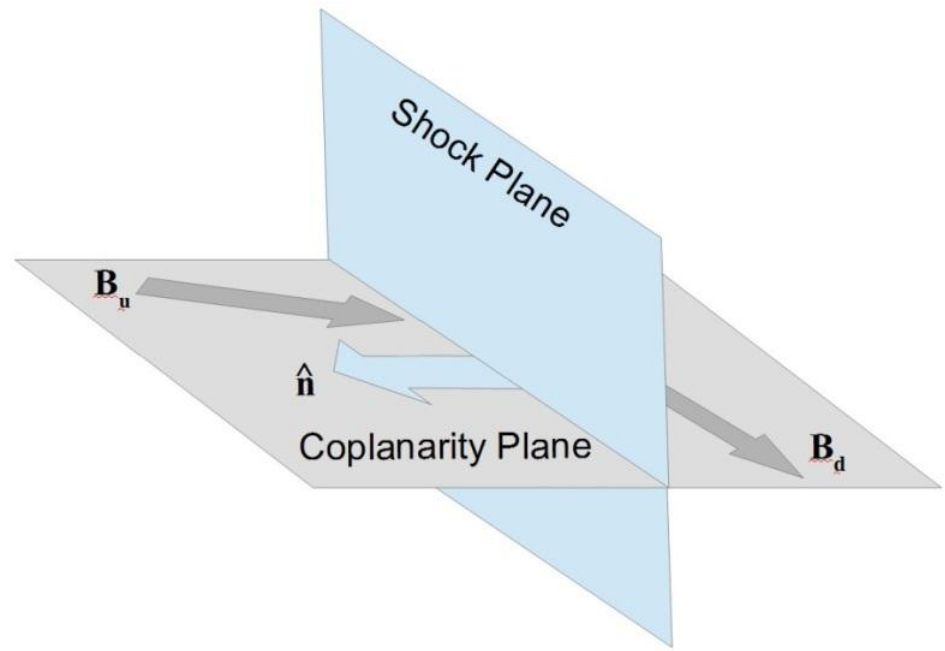

Figure 4.1.: Diagram illustrating the coplanarity plane which intersects the shock front perpendicularly. This plane contains the upstream and downstream magnetic field vectors and the local shock normal.

It is obvious that equation 4.3 breaks down at $\theta_{B n}=0^{\circ}$ and $90^{\circ}$. While these two extreme cases are uncommon, practical limitations still prevail. Magnetic field measurements downstream are characterised by large fluctuations and this yields a substantial error associated with measuring $\boldsymbol{B}_{\boldsymbol{d}}$. The closeness of the measured $\boldsymbol{B}_{\boldsymbol{d}}$ to representing the actual field downstream depends on the interval over which the data is averaged. Doubts in selecting a downstream interval stem from deciding what really is representative of the region "immediately downstream" given that the shock is in continuous motion and whether the selection criterion is consistent throughout all shock crossings. Moreover, for quasiparallel $\left(\theta_{B n}<45^{\circ}\right)$ configurations, this difficulty extends to measurements of $\boldsymbol{B}_{\boldsymbol{u}}$ where there are large fluctuations $\left(\delta \mathbf{B} / \mathrm{B}_{0} \sim 1\right)$ associated with the foreshock re- 
gion. This technique is nevertheless broadly used particularly in studies on one or very few shock crossings [e.g. Achilleos et al., 2006].

The second and preferred technique, as far as this thesis is concerned, uses the model boundary equation from W11. The equation describing the size and shape of Saturn's bow shock is that of a cylindrically symmetric conic section parameterised by upstream conditions using over 500 crossings. The distance of the subsolar point, $R_{S N}$, was assumed to vary with the upstream dynamic pressure, $P_{S W}$, according to a power law that was determined empirically.

$$
R_{S N}=c_{1} P_{S W} \frac{-1}{c_{2}}
$$

where $c_{1}=15 \pm 1 \mathrm{R}_{\mathrm{s}}$ and $c_{2}=5.4 \pm 0.5$. The equation of the bow shock surface in polar coordinates expressed in polar form is

$$
r(\theta)=\frac{(1+\varepsilon) R_{S N}}{1+\varepsilon \cos \theta} ; \varepsilon=0.84
$$

Figure 4.2 is an example of the model for a median subsolar distance of 27 $\mathrm{R}_{\mathrm{S}}$. It is worth noting that the direction of the solar wind $V_{S W}$ is in the planet's rest frame to account for Saturn's orbital speed of $9.7 \mathrm{~km} / \mathrm{s}$. The angle corrected is small at $\sim 2^{\circ}$ (c.f. Earth at $\sim 4^{\circ}$ ) and this is known as aberration. Here the normal is determined geometrically from knowledge of the spacecraft position only. The position is written in the form $(r, \theta, \varphi)$ where $r$ is the planetocentric distance to the point on a surface, $\theta=\cos ^{-1} X_{k s m} / r$ and $\varphi=\tan ^{-1} Z_{K S M} / Y_{K S M}$. The normal is then given by

$$
\boldsymbol{n}_{\boldsymbol{M}}=\frac{\partial \boldsymbol{r}}{\partial \theta} \times \frac{\partial \boldsymbol{r}}{\partial \varphi}
$$




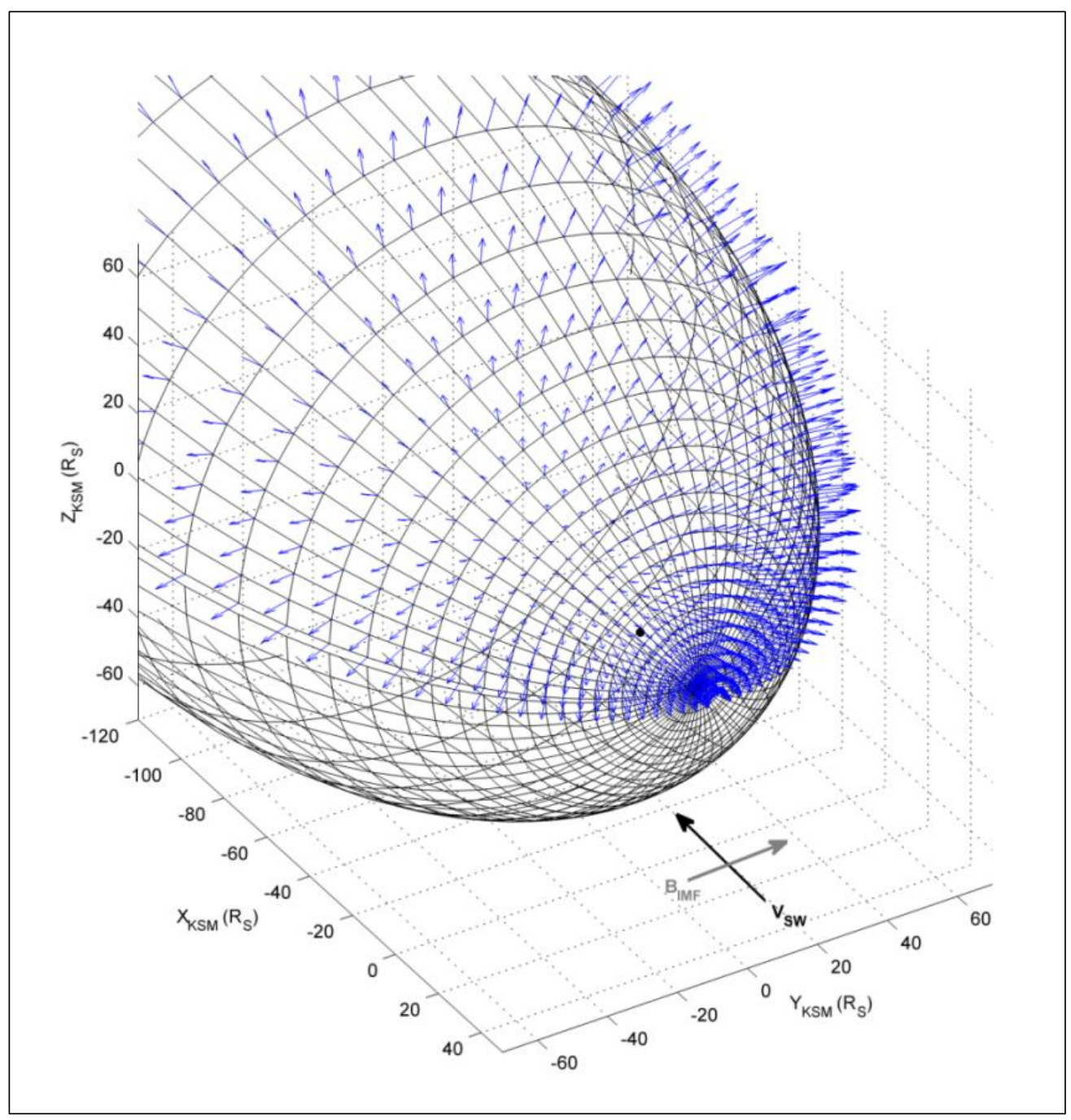

Figure 4.2.: The most up-to-date 3D bow shock model (W11) with surface described by the equation of a conic section. The direction of the solar wind is indicated as $V_{S W}$ with the IMF in a typical orientation of roughly perpendicular to $V_{S W}$ in the ecliptic plane. Blue arrows on the northern $\left(+Z_{K S M}\right)$ represent the local normals to the surface.

Horbury et al., (2002) estimated the local normals of 48 quasiperpendicular shock crossings at Earth using Cluster's fleet of four spacecraft. They compared the normals, determined using inter-spacecraft timings, with each of those estimated using the Coplanarity Theorem and a bow shock model from which the methodology of W11 was inherited [Formisano, 1979; Peredo et al. 1995]. They found large discrepancies of $22^{\circ} \pm 4^{\circ}$ with the Coplanarity Theorem. The comparison with the model, on the other hand, was found to be in re- 
markably good agreement with $\sim 80 \%$ of the sample having a deviation of less than $10^{\circ}$.

\subsection{Characterisation of Saturn's Bow Shock}

While shock waves can be characterised using several Mach numbers, we will use the Alfvén Mach number, $M_{A}$, in this thesis. Like most studies, $M_{A}$ is the preferred choice of Mach numbers since it is the easiest to compute. The fast magnetosonic speed needed to determine the fast Mach number, $M_{f}$, is complicated by the non-isotropic nature of MHD modes and thus dependent on the propagation direction upstream, $\theta_{B n}$. Determination of $M_{A}$ does not require this parameter nor temperature measurements (a difficulty specific to Cassini). Nevertheless, the limited plasma datasets have not undermined in-depth studies of the physical properties Saturn's bow shock and neighbouring regions [Achilleos et al., 2006; Masters et al., 2011; Masters et al., 2013].

Another controlling parameter would be the plasma $\beta$. For the aforementioned reasons, it is very difficult, and not possible in many cases, to obtain its values upstream. Separating the effects of the different Mach numbers and $\beta$ remains unclear even with the most extensive study using the most sophisticated techniques to date [e.g. Henley, 2010]. This is because these quantities are all interdependent and thus generally change together. This predicament is reflected in the literature where different authors conclude various degrees of importance these quantities have on the shock's character.

Previous works have studied the overshoot in the magnitude of the magnetic field - a key diagnostic of the role of ion dynamics - as a function of Mach numbers and $\beta$. Russell et al. (1982) found the overshoot magnitude to increase with both $M_{f}$ and $\beta$. Mellott and Livesey (1987) revisited this problem and found that the overshoots are controlled principally by $M_{A}$ and to a lesser extent by $\beta_{i}$ and $\beta_{e}$. They suggested that the large overshoots seen by Russell et al. (1982) can be explained by so-called turbulence induced at higher Mach numbers. 
The interlinking of $M_{A}$ and $\beta$ can be shown by algebraic manipulation since both depend on the upstream magnetic field. From $M_{A}{ }^{2}=P_{d y n} / 2 P_{m a g}$ and $\beta=$ $P_{t h} / P_{\text {mag }}$, we can write

$$
\beta_{i(e)}=T_{i(e)}\left(\frac{2 k_{B}}{m_{i(e)} u^{2}}\right) M_{A}^{2}
$$

Equation 4.7 shows the relationship between $M_{A}{ }^{2}$ and $\beta$. The subscripts $i$ and $e$ denote the ion and electron species respectively. In reality, the temperature $T$ and inflow speed in the shock rest frame $u=\boldsymbol{u} \cdot \widehat{\boldsymbol{n}}$ varies between shock crossings. For a large enough sample, however, we expect to see this correlation. Henley (2010) has meticulously worked on characterising Earth's bow shock and showed a high correlation of $r^{2}=0.71$ between $M_{A}{ }^{2}$ and $\beta$ for 75 wellcharacterised shocks used (see Figure 4.3).

For a large sample (e.g. $>800$ shock crossings in Figure 4.4), regions of low (high) Mach numbers are likely to correspond to low (high) $\beta$. It is, however, crucially important to keep in mind that correlation does not add to the evidence that a causal link may exist. For example, while positive correlations of magnetic overshoots with $\beta$ have been widely reported, it remains unclear whether there is truly an independent $\beta$ effect. Another important point to be mindful of is that defining the magnetic overshoot (which requires the maximum field $B_{\max }$ ) is related to the resolution of the data.

In this work, we have used data from the years 2004-2012 with 871 identified shock crossings. These do not include immediately successive crossings so as not to create a bias towards crossings in the same location of a parameter space. Figure 4.4 highlights the spacecraft positions where bow shock crossings were identified relative to Saturn at the origin. A vast majority of the coverage is on the dayside, particularly limited to regions of low to mid-latitudes and roughly equal on dawn and dusk flanks. The magnetic field measurements used are at $1 \mathrm{~s}$ resolution. 


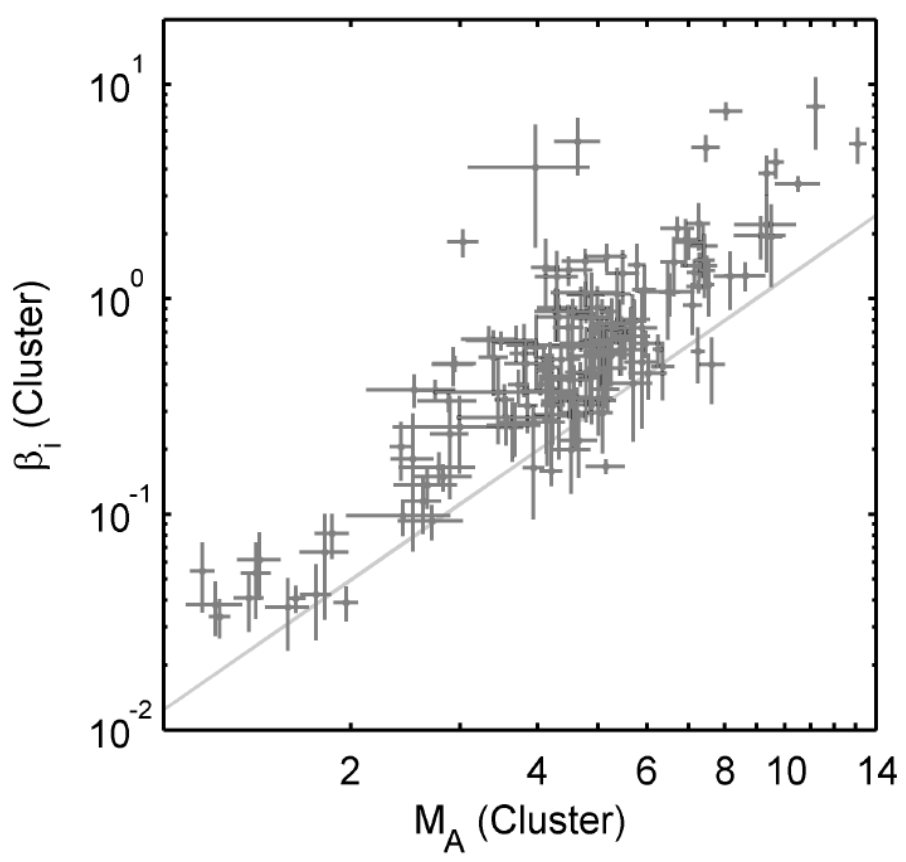

Figure 4.3.: Correlation of $\mathrm{M}_{\mathrm{A}}$ and $\beta$. Each marker represents a shock crossing with error bars They grey line indicates the functional form expected for a typical ion temperature of $1.2 \times 10^{5} \mathrm{~K}$ and upstream velocity of $400 \mathrm{~km} / \mathrm{s}$. Figure taken from Henley (2010).

\subsubsection{Determination of the Alfvén Mach Number}

The Alfvén Mach number, $M_{A}$, is the key parameter in organising the large sample of shock crossings into regimes in a parameter space. In this way, we are able to focus on a subset as a particular class of shocks and draw comparisons. Recall this quantity is given by

$$
M_{A} \equiv \frac{u}{v_{A}}=\frac{\sqrt{\mu_{0} P_{d y n}}}{B_{u}}
$$

where $P_{d y n}$ is the upstream ram pressure along the shock normal $\rho u^{2} \cos ^{2} \theta_{u n}, \theta_{u n}$ is the angle between the upstream flow vector $\boldsymbol{u}$, assumed to always be directed along $-X_{K S M}$, and the local normal of the shock surface $\widehat{\boldsymbol{n}}$. The ram pressure is estimated from the power law in Equation 4.4 based on the crossing position of 


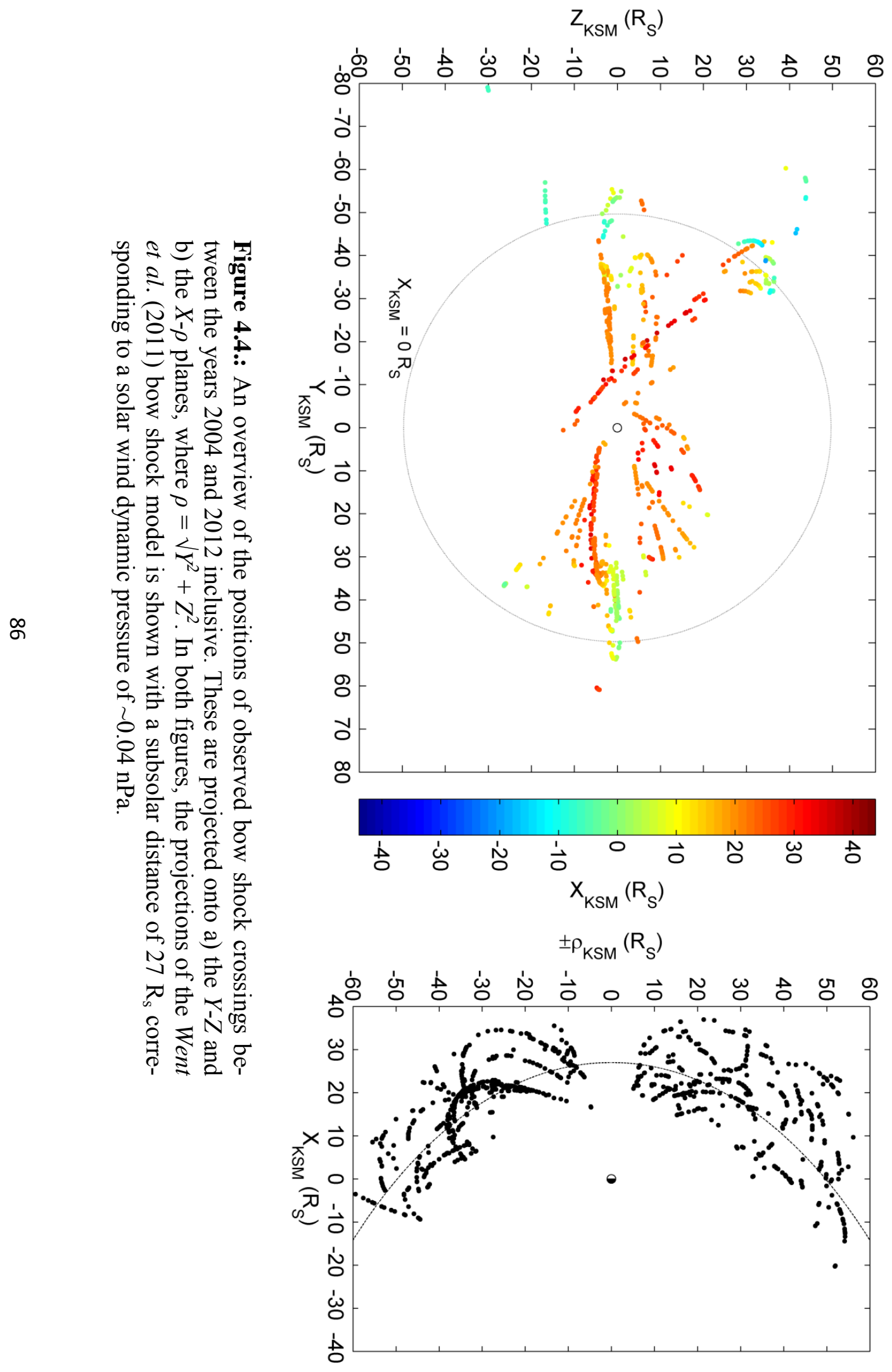


each crossing. The associated errors are from the empirically determined $c_{1}$ and $c_{2}$ and are small. Embedded in this relationship are local density measurements and solar wind propagations. By obtaining $P_{d y n}$ and $B_{u}$ for each crossing, we are then able to calculate $M_{A}$ using Equation 4.8.

\subsection{Parameter Space}

With these estimations, a parameter space can be constructed to show the distribution of $M_{A}$ (see Figure 4.5). The median $M_{A}$ of 14 (red dashed line) is indeed close to the theoretical expectation from Figure 2.10. The crossings span across two orders of magnitude of $M_{A}$ from an Earth-like regime of 2-8 to an astrophysical-like regime of $O(10)-O\left(10^{2}\right)$. It is not only evident from Figure 4.5 that the typical $M_{A}$ is higher at Saturn but also that the environment is more variable.

Three quasi-perpendicular shock crossings of different $M_{A}$ are represented as red markers on the parameter space and the differences in their magnetic field profiles are very obvious in Figure 4.6. This particular set of crossings (increasing $M_{A}$ and similar $\theta_{B n}$ ) was chosen to compare a pair of similar $B_{u}$ and a pair of similar $P_{d y n}$. The first is an inbound (i.e. passing from upstream to downstream) crossing of $M_{A} \sim 5$ and is one typically found in the near-Earth space. It is characterised by a sharp, local transition between both regimes - a feature unique to quasi-perpendicular shocks. As $M_{A}$ increases in the second and third panels, $\sim 22$ and $\sim 38$ respectively, there is an increasing trend in the downstream variability, maximum field and prominence of the foot region preceding the ramp. Here, ion dynamics becomes important and this is discussed further in Chapter 5. 


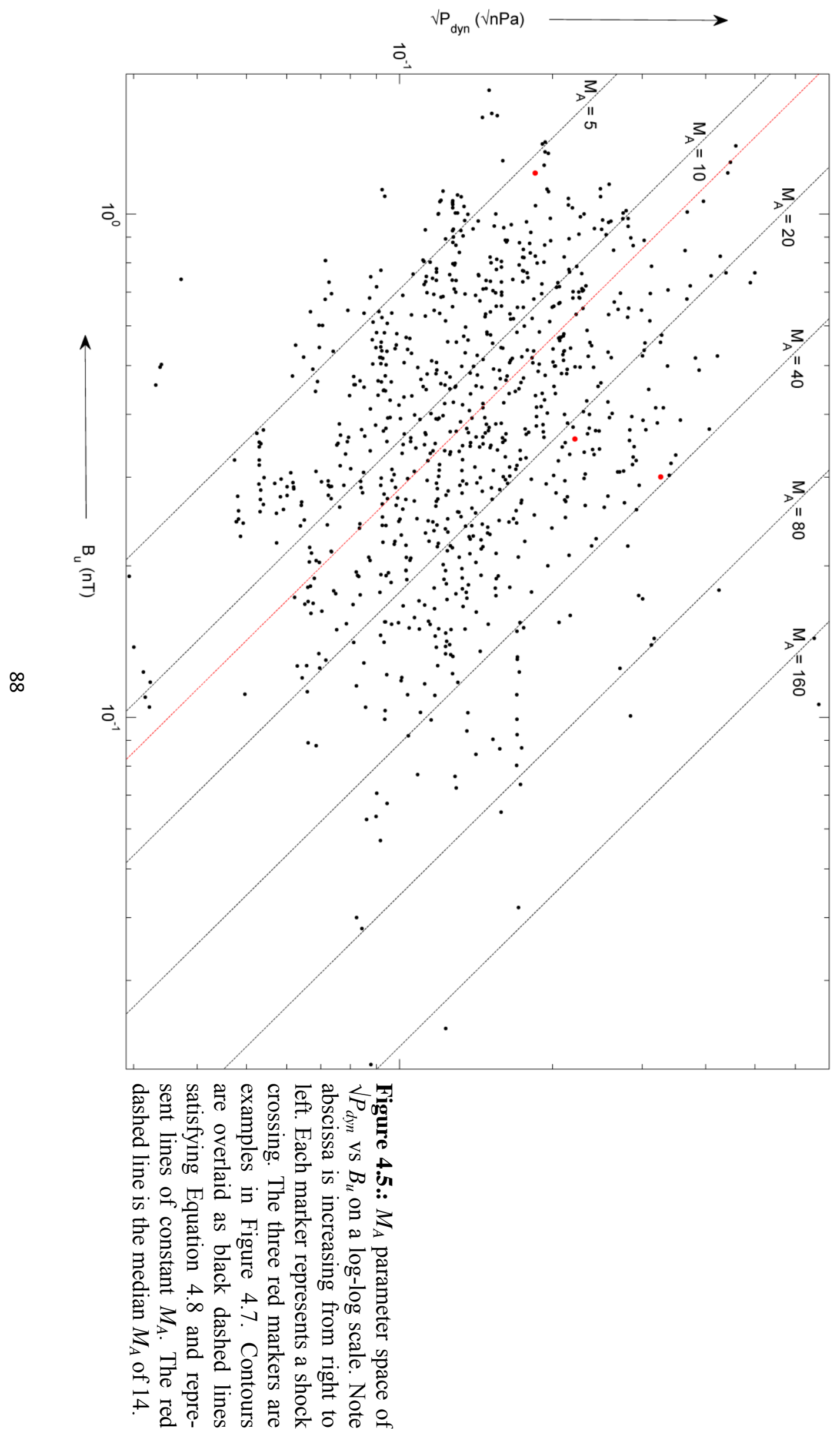



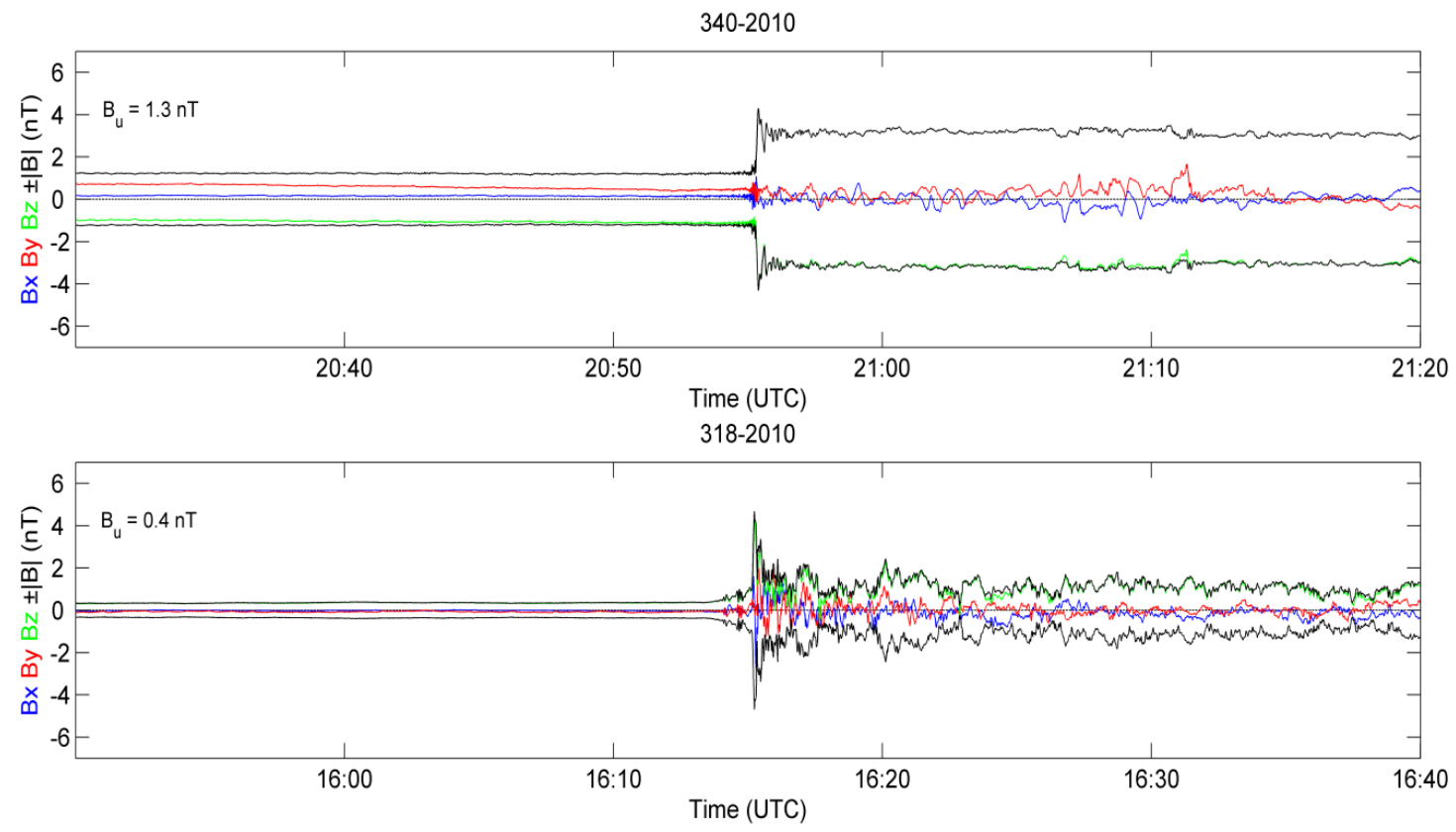

071-2007

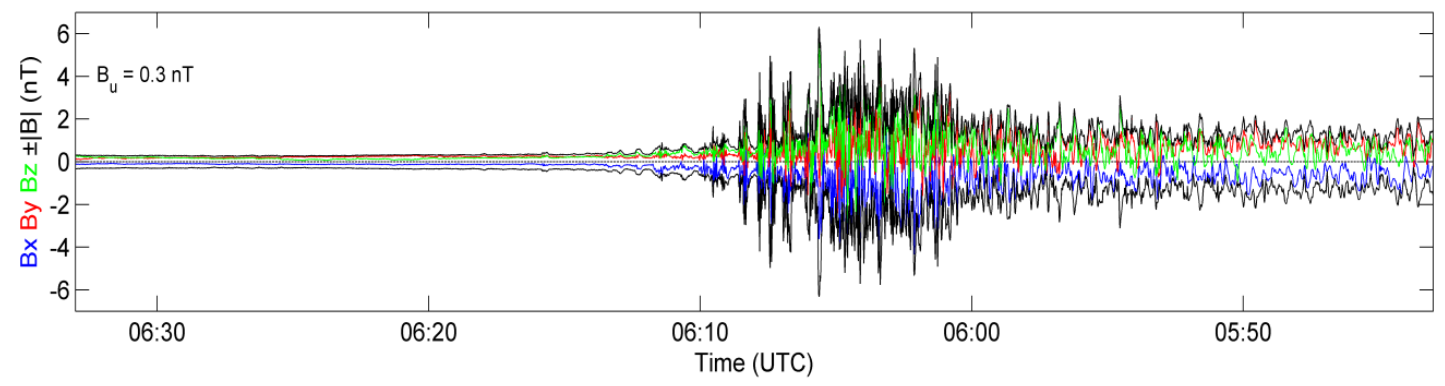

Figure 4.6.: Three magnetic field time series of quasi-perpendicular shock crossings of increasing $M_{A}$ corresponding to the three red markers on Figure 4.6. From top to bottom: $M_{A} \sim 5 \& \theta_{b n}=65^{\circ}, M_{A} \sim 22 \& \theta_{b n}=79^{\circ}$ and $M_{A} \sim 38$ $\& \theta_{b n}=79^{\circ}$. The top two panels are inbound crossings. The bottom panel is an outbound panel with the time series reversed. 
Figure 4.7 (a-d) summarises the sample of crossings into four distributions of $\theta_{B n}, B_{u}$, standoff distance $R_{b s}$ and $M_{A}$. Figure 4.7 a reveals that the quasiperpendicular configuration is the most observed at Saturn's bow shock with $9 \%$ for $\theta_{B n}<45^{\circ}, 81 \%$ for $\theta_{B n} \geq 45^{\circ}$ and $50 \%$ for $\theta_{B n} \geq 70^{\circ}$. This is attributed to the Parker spiral at 10 AU being significantly more azimuthal. Another factor is the orbit of Cassini which crosses the bow shock mostly in the dayside where the surface is blunt. For these reasons, we expect the detection of a foreshock region to be uncommon though this has been reported nonetheless [Bertucci et al., 2007; Andrés et al., 2013]. Another possibility for this could be from the fluctuations in the IMF. We expect the favourable IMF direction to remain steady long enough for a foreshock to be set up and detected far upstream by Cassini. This is beyond the scope of this thesis; however a study of $\delta \mathbf{B}(\mathrm{t})$ upstream of Saturn is a topic that would likely be pursued in the future.
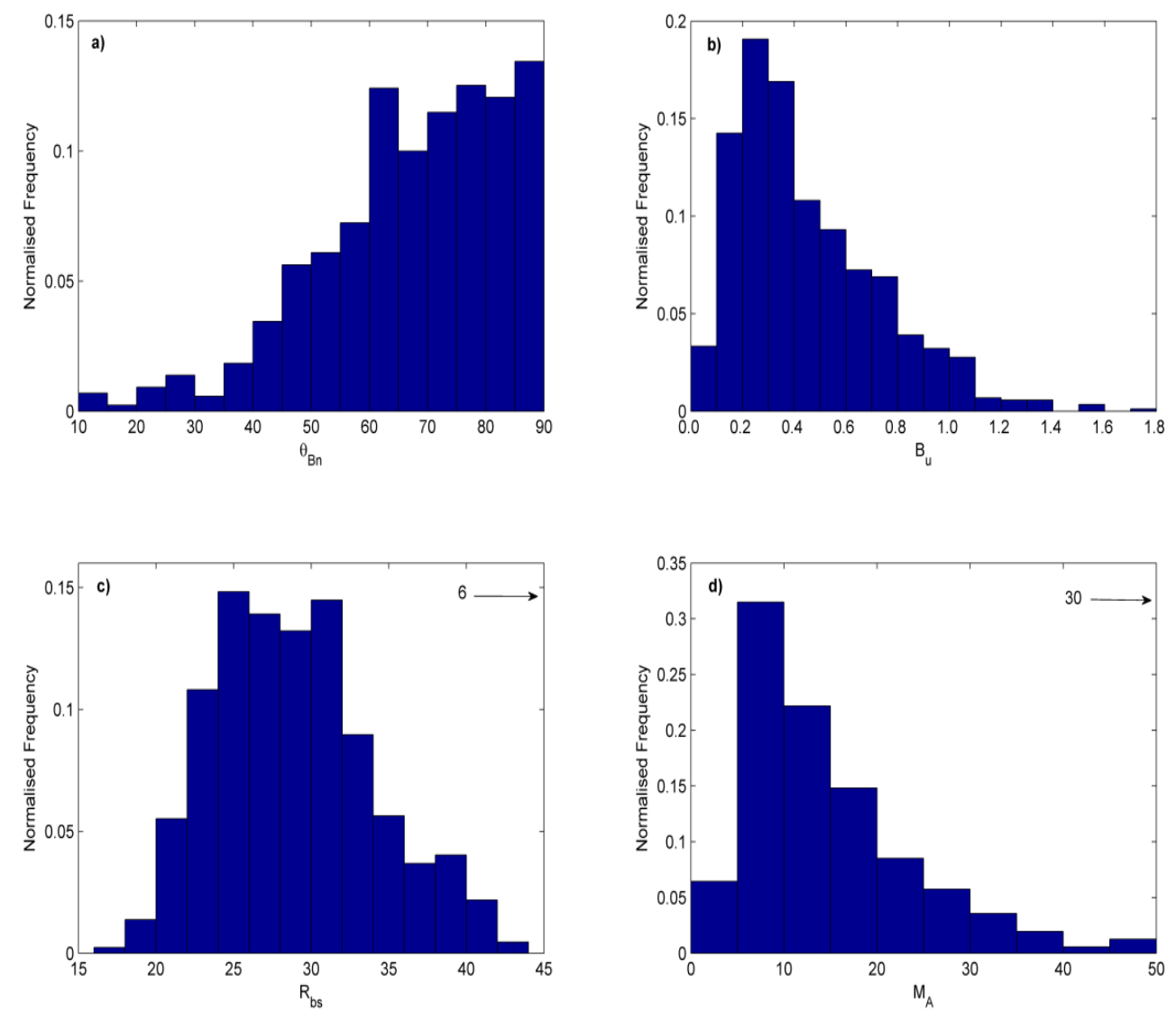

Figure 4.7.: Normalised frequency distributions of all (871) crossings of Saturn's bow shock for a) $\theta_{B n}$ with bin width of $5^{\circ}$ b) $B_{u}$ with bin width of $0.1 \mathrm{nT}$ c) standoff distance $R_{b s}$ with bin width of $2 R_{S}$ and d) $M_{A}$ with bin width of 5 . 
The two peaks in the distribution of bow shock standoff distances correlate with the distribution of magnetopause standoff distances [Achilleos et al., 2008] and this has been attributed to the two states of Saturn's loading-unloading magnetosphere cycle [Rymer et al., 2013]. The magnetopause peaks at $\sim 22$ and $\sim 27$ $\mathrm{R}_{\mathrm{S}}$ while the bow shock peaks at $\sim 26$ and $31 \mathrm{R}_{\mathrm{S}}$. Subtracting the peaks of the two boundaries gives a magnetosheath subsolar thickness of $\sim 4 \mathrm{R}_{\mathrm{S}}$. Recalling that the standoff distance is used as a proxy for the upstream ram pressure, the distribution in $M_{A}$ is principally controlled by that of $B_{u}$. This is because the spread in $B_{u}$ spans two orders of magnitude upstream of Saturn's bow shock compared with $\rho u^{2}$ varying by only one order of magnitude. Another reason is because the $\mathrm{M}_{\mathrm{A}}$ changes as $1 / B_{u}$ and only $\sqrt{ } \rho u^{2}$. The largest Mach numbers, in particular, are much more likely to be attributed to very low IMF strengths rather than unusually large ram pressures (e.g. arrival of ICMEs). Compared to Earth, for the Alfvén speed (since $v_{A} \alpha 1 / B$ ), the magnetic field strength is much lower upstream of Saturn thus a small change in the quantity yields a very large change in the $v_{A}$. For example, a typical $B_{u}$ of $4 \mathrm{nT}$ at Earth requires a change of $2 \mathrm{nT}$ for a $50 \%$ change in $M_{A}$, whereas at Saturn a typical $B_{u}$ of $0.6 \mathrm{nT}$ requires a change of 0.3 nT for the same change in $M_{A}$.

\subsection{Shock Relative Overshoot and Variability}

It has been established that for super-critical quasi-perpendicular collisionless shocks, microphysics must be invoked to account for the deficit in dissipation that cannot be accommodated by the hydromagnetic formalism i.e. the Rankine-Hugoniot equations. The shock overshoot is a unique and important feature of such shocks immediately succeeding the ramp with a length scale in the order of an ion gyroradius. One of the earliest detections of the shock overshoot by Russell and Greenstadt (1979) highlighted that ion thermalisation takes place within the ramp and completes only after the overshoot has finished. Far downstream from this region and in steady-state, the magnetic field returns to its Rankine-Hugoniot predicted value. 
The overshoot for each crossing can be quantified in a number of ways and here we choose to be consistent with the widely used Relative Overshoot Amplitude (ROA) which is given by

$$
R O A=\frac{B_{\max }-\left\langle B_{d}\right\rangle}{\left\langle B_{d}\right\rangle}
$$

where $B_{\max }$ is the highest field strength recorded in the crossing and $B_{d}$ is the downstream field. This is averaged over an interval far enough from the overshoot-undershoot region but close enough to the shock ramp to give a better representation of the downstream magnetic field strength.

Figure 4.8a presents the ROA calculations for quasi-perpendicular shocks as a function of $M_{A}$. Table 4.1 provides a summary of the corresponding statistical measures. It is clear at first from Figure $4.8 \mathrm{a}$ that the super-criticality of Saturn's bow shock prevails with all crossings having a positive non-zero ROA. The positive correlation here is moderate-to-strong and the unprecedented range of $M_{A}$ and sample size may confirm that the Mach number is indeed the principal controller of the overshoot. The view here leans much more towards the $M_{A}$ dependence argument, in spite of the absence of $\beta$ measurements. While $\beta$ and $M_{A}$ are interlinked and we expect on average the high (low) $M_{A}$ to be associated with higher (lower) $\beta$, the range of $\beta$ is significantly shorter than that of $M_{A}$ in the solar wind near Saturn [Jackman and Arridge, 2006]. More convincingly from a theoretical perspective, the Mach number takes into account the speed of the flow which in a sense is a measure of the mass flux that requires dissipation across the shock. The Mach number can be arbitrary and hence there is no upper limit on how much mass flux enters the shock to subsequently be dissipated by anomalous resistivity and particle trajectory; ensuring the adequately heated and decelerated flux downstream for a sub-fast magnetosonic regime. Since the Rankine-Hugoniot equations dictate a maximum field enhancement downstream of 4 (assuming $\gamma=5 / 3$ for a perpendicular shock) for the conservations law to be maintained, any increase above this limit must be attributed to an additional processes beyond the classical fluid framework. As a result, increases in the Mach 
number must intensify the role of such additional processes, one of which is manifested as the overshoot.

Figure $4.8 \mathrm{c}$, on the other hand, shows no clear correlation between ROA and $\theta_{B n}$. This is broadly consistent with the simulation runs by Tiu et al. (2011) where they concluded that the overshoot is insensitive to $\theta_{B n}$. $\theta_{B n}$ is a geometric rather than a physical factor like the Mach number or $\beta$. In the context of its role as a control parameter, changes in this quantity (assuming all other parameters are held fixed), affect only the pathway the ions take to achieve the required thermalisation. More on this will be discussed in Chapter 5 .
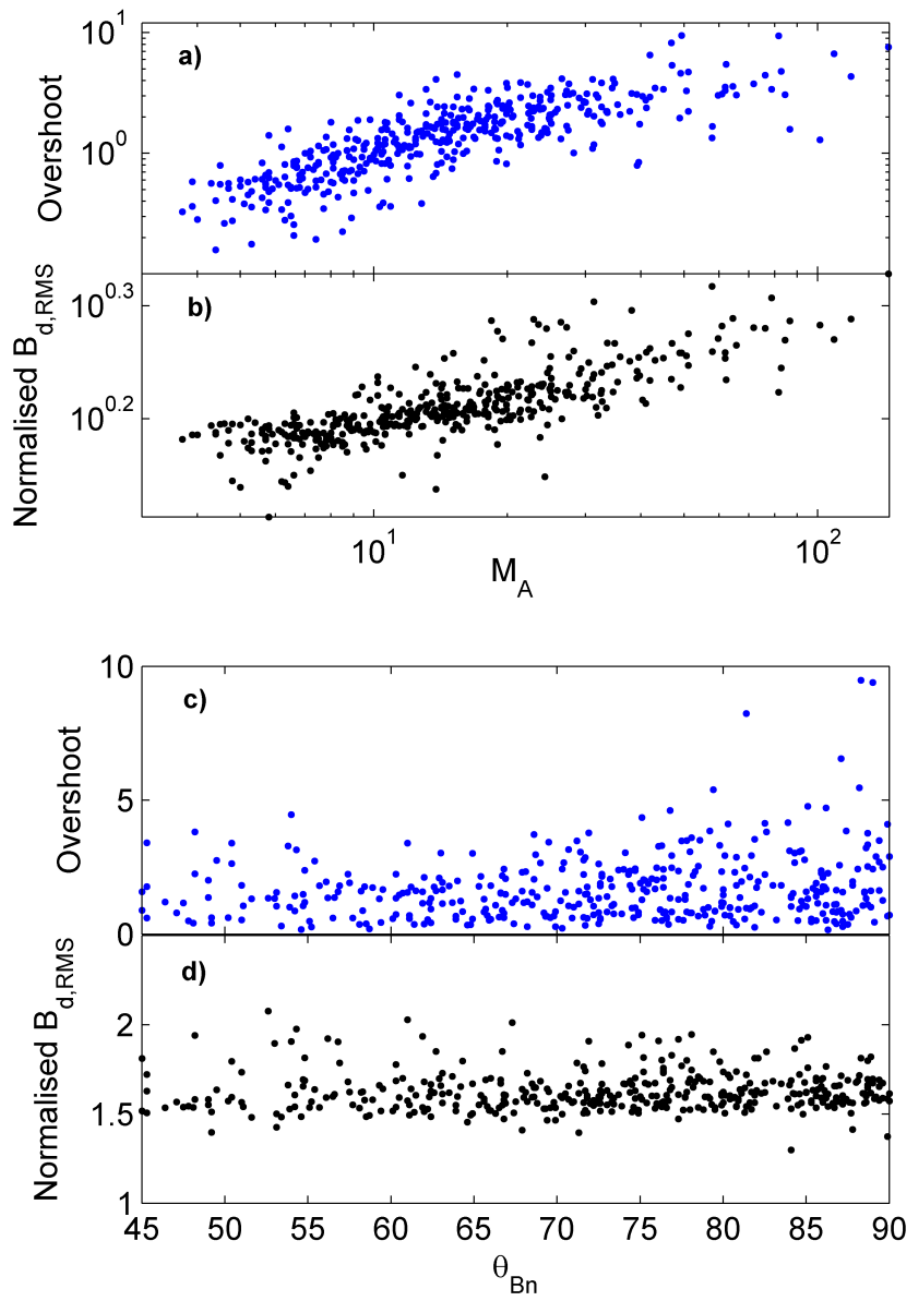

Figure 4.8.: (a) Overshoot and (b) variability plotted against $M_{A}$ for highly quasiperpendicular shocks $\theta_{B n} \geq 70$. (c) Overshoot and (d) variability plotted against $\theta_{B n}$. 


\begin{tabular}{cccc}
\hline Panels & $r^{2}$ & $m$ & $N$ \\
\hline $\mathrm{a}$ & 0.60 & 0.82 & 422 \\
$\mathrm{~b}$ & 0.61 & 0.08 & 422 \\
$\mathrm{c}$ & 0.01 & 0.08 & 422 \\
$\mathrm{~d}$ & 0.01 & -0.02 & 422 \\
\hline
\end{tabular}

Table 4.1.: Statistical measures of the scatter plots on Figure 4.9. $r^{2}$ is the coefficient of determination (range $0 \leq \mathrm{r}^{2} \leq 1$ ), $m$ is the slope of the best-fit line (log$\log$ scale) and $N$ is the sample size.

The variability downstream is also quantified using the root-mean-square (RMS) value of the same downstream interval normalised by the average of the upstream and downstream magnetic field magnitudes (away from the foot and overshoot-undershoot regions). This is given by

$$
B_{\text {ave }}=\frac{1}{2}\left(\left\langle B_{u}\right\rangle+\left\langle B_{d}\right\rangle\right)
$$

Equation 4.10 corrects for the fact that two shocks can have the same $M_{A}$ and $\theta_{b n}$ but different $B_{u}$ and transition profiles thus allowing for better comparison of underlying trends with the control parameters.

Figure $4.8 \mathrm{~b}$ presents the normalised downstream RMS as a function of $M_{A}$ for the same quasi-perpendicular shocks. It is clear that with increasing $M_{A}$ the variability in the signal becomes more pronounced. The variability is interpreted as spatio-temporal substructures which can be used as an indicator of a shock's departure from a one-dimensional surface. What is observed could possibly be a combination of scaled length effects [Bale et al., 2003; Scholer and Burgess, 2006], rates of change of the structure and time spent within the shock layer [Burgess et al., 2006]. We expect as a result some observational bias in the data, for example low vs high speed shock crossings.

Again, Figure 4.8d reveals no obvious correlation between the normalised downstream RMS and $\theta_{B n}$. While the full range of $\theta_{B n}$ is not shown, the variability is expected to be more pronounced when $\theta_{B n}$ is less than $45^{\circ}$. This is due to the generation of wave structures in a quasi-parallel regime. As for the quasi- 
perpendicular regime, the argument for the lack of a visible trend in Figure 4.8d is probably the same as stated for Figure $4.8 \mathrm{c}$.

\subsection{Conclusions}

- We have accumulated a very large sample of Saturn's bow shock crossings and have presented distributions of the upstream conditions. Most of the crossings were in a quasi-perpendicular configuration by virtue of a combination of the Parker spiral at $10 \mathrm{AU}$ and the location of the crossings taking place on the dayside of Saturn.

- Using the upstream magnetic field strength and estimated dynamic pressure of each crossing, the Alfvén Mach number was able to be obtained. This technique, presented here for the first time, has the advantage of overcoming the difficulty and limitations of Cassini's plasma instrument to obtain a Mach number for each of the 871 shock crossings, as presented in Figure 4.5. This has enabled a study of the overall characteristic of Saturn's bow shock and, more interestingly, laid the foundation for a natural follow-up detailed study of the highest Mach number regime as presented in Chapter 5.

- The Alfvén Mach numbers determined show a median value significantly higher than at Earth $\left(M_{A}=14\right)$ and the largest range seen at any planet, owing to the upstream magnetic field.

- The magnetic overshoot was shown to correlate quite strongly with $M_{A}$ across the entire range and we conclude that it is most likely the primary controller. Although a $\beta$ dependence has been reported, the absence of the parameter in this study is not likely to make it any less instructive since its range is similar at all planets [Russell et al., 1982]. 


\section{Quasi-perpendicular High Mach Number Collisionless Shocks}

\subsection{Introduction}

Chapter 4 presented the overall magnetic structure of Saturn's bow shock and emphasised the wide range of $M_{A}$ available at $10 \mathrm{AU}$. This chapter focusses on the poorly explored regime of $M_{A} \geq 25$ ( $80^{\text {th }}$ percentile), where in situ observations have been few and far between. Within this subset, a noticeable number of distinct crossings appear, exhibiting features unlike the "textbook" quasi-perpendicular shocks (e.g. top panel in Figure 4.7).

Recall from Chapters 1 and 4 that observations and simulations show supercritical shocks compensate for the shortfall in dissipation by reflecting some fraction of the incoming ions back upstream [Treumann, (2009)]. At such high Mach number shocks, the structure becomes inherently dependent on the ion dynamics, and a fluid description is inadequate. Although ion reflection dominates heating at high Mach number shocks, in recent years, attention has focused on variability or nonstationarity in the shock structure at ion timescales. There are several proposed mechanisms, mostly based on simulations and theoretical considerations [Biskamp and Welter, 1972; Lembege and Dawson, 1987; Quest, 1985]. At sufficiently high Mach number and low upstream ion beta $\beta_{i}$, there is a quasi-periodic, cyclic reformation of the shock associated with overreflection of ions [Morse et al., 1972; Hellinger et al., 2002]. This mechanism predicts a timescale for reformation of the order of the ion cyclotron period [Chapman et al., 2005]. Nonstationarity has also been suggested to be the outcome of a gradient catastrophe of nonlinear upstream whistler, associated with Mach numbers greater than the (nonlinear) whistler critical Mach number beyond which an upstream whistler cannot phase stand in the upstream flow 
[Krasnoselskikh et al., 2002]. An alternative mechanism found in particle-in-cell (PIC) simulations is the quasi-periodic disruption of the ion foot due to the modified two-stream instability [Scholer and Matsukiyo, 2004].

Shock reformation has primarily been studied using hybrid and PIC simulations, where a comprehensive picture of the time evolution of the shock structure is available. In situ spacecraft observations have neither been frequent [Bagenal et al., 1987] nor extensive enough to corroborate these studies. This is mainly due to most studies of shock crossings being near the Earth where the Mach numbers typically range from low to modest $\left(M_{A}=2-8\right)$. While some observations have been reported at Earth's bow shock [Lobzin et al., 2007], they remain open to interpretation [Comisel et al., 2011].

\subsection{Method}

Data was analysed mainly from the magnetometer and use the plasma instruments, the Radio and Plasma Wave Science (RPWS) [Gurnett et al., 2004] and the Ion Mass Spectrometer (CAPS-IMS) [Young et al., 2004]. Since the focal point of this study is a much smaller sample size of crossings, the Mach numbers reported from Equation 4.8's estimation are validated using ion densities and solar wind speeds (in the shock's rest frame) from the plasma instruments where both observations are available and unambiguous. Figure 5.1 shows the estimations are in very good agreement with the observations.

Figure 5.1.: Observed $M_{A}$ vs W11 model. Dashed line is $y=x$.

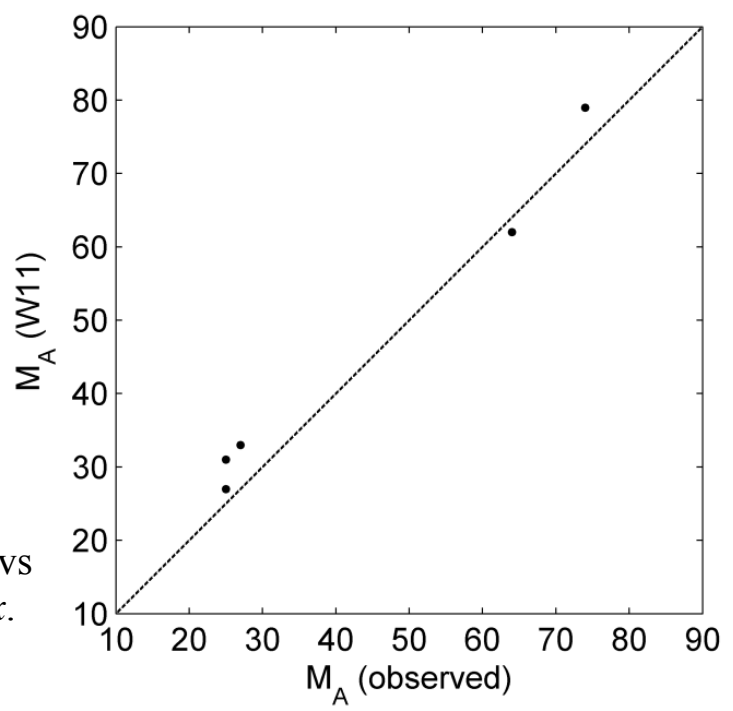


The errors associated with both the observed and W11 Mach numbers primarily originate from the W11 model. As will be shown in $\$ 5.2 .1, \theta_{B n}$ is required for estimating the shock speed to convert the observed upstream speed from the spacecraft to the shock frame. Determination of $\theta_{B n}$ uses the W11 model normal. However as discussed in Chapter 4, the errors are not only small but shock models are very reliable as confirmed by Horbury et al. (2002) with multipoint spacecraft observations.

\subsubsection{Determination of the Foot Length and Shock Speed}

As pointed out earlier, a single spacecraft presents the challenge of distinguishing spatial and temporal dependences which includes the inference of shock speeds. Thomsen and Gosling (1985) demonstrated a formalism to estimate the shock speed by measuring the time it takes for the foot to convect across the spacecraft. Here, the length of the foot is determined from an expression for the turnaround distance of an incident ion that is specularly reflected at the shock. This is motivated by the fact that the foot of a quasiperpendicular, supercritical shock is spatially coincident with gyrating ions at the shock [Achilleos et al., 2006]. This distance, $d$, is given by

$$
d=\frac{V_{u}}{\Omega_{C}}\left[\psi\left(2 \cos ^{2} \theta_{B n}-1\right)+2 \sin ^{2} \sin \psi\right]
$$

where $V_{u}$ is the incident ion velocity parallel to the shock normal. This is derived from CAPS-IMS when the upstream flow is in the instrument's field-of-view. $\Omega_{c}$ and $\theta_{B n}$ are the proton angular gyrofrequency and contact angle respectively. $\psi$ is evaluated as

$$
\cos \psi=\frac{1-2 \cos ^{2} \theta_{B n}}{2 \sin ^{2} \theta_{B n}}
$$

As this model describes an ion's reflection trajectory, Equation 5.1 has no real solutions for $\theta_{B n}<30^{\circ}$. Under these highly quasi-parallel configurations, the 
ions do not re-encounter the shock upon reflection and escape upstream. From $\theta_{B n}$ of $30^{\circ}$ to $90^{\circ}$ (perfectly perpendicular), the term multiplying the upstream convective gyroradius $\left(V_{u} / \Omega_{c}\right)$ decreases from 1.57 to 0.68 . It is important to note that this model is not representative of the complex reflection process as simulations suggest. Nevertheless, it is shown to be broadly consistent with observations and thus a good approximation [Newbury and Russell, (1996)].

The relationship between the shock speed, $u_{s}$, along the shock normal and the convection of the foot is hence given by

$$
\frac{d}{\Delta t}=\left|\boldsymbol{u}_{s \boldsymbol{c}} \cdot \boldsymbol{n}-u_{s}\right|
$$

where the spacecraft speed, $u_{s c}$, is measured in the KSM coordinate system. Figure 5.2 shows how the transit time, $\Delta t=\left|t_{r}-t_{u}\right|$, is estimated from the magnetic field time series; where $t_{u}$ is when the upstream field magnitude exceeds four standard deviations (added to the mean) and $t_{r}$ is when a linear function fitting the ramp intersects this threshold. This is a standard practice of quantitatively identifying the foot region [e.g. Achilleos et al., 2006].

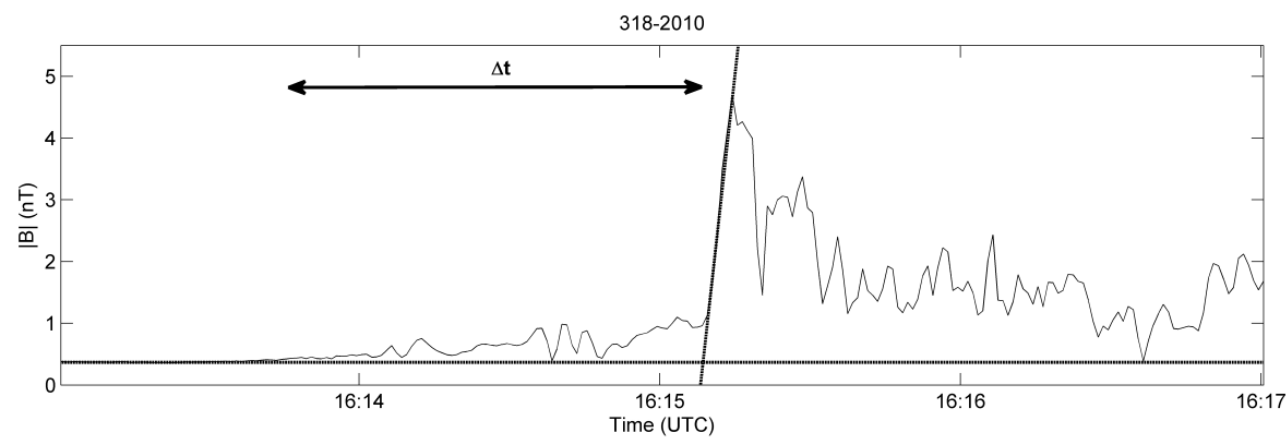

Figure 5.2.: Magnetic field time series of a quasi-perpendicular shock crossing. The horizontal dashed line is the mean magnetic field upstream and the slanted dashed line is a linear function fitting the ramp. The double headed arrow is the time between $t_{u}$ and $t_{r}$ as defined in the text. 


\subsection{Observations}

Figure 5.3 (a-c) show three examples of quasi-perpendicular crossings from upstream of the shock to downstream (right to left) using magnetic field data. These are three examples revealing the foot signatures, of enhanced magnetic field strength upstream, occurring at regular intervals suggestive of reformation cycles (with the frequency also present in the downstream magnetic field). These pulses are attributed to temporal variations of the reflected fraction of the incident solar wind. In addition, the overshoots of these crossings are enhanced relative to the upstream magnetic field by a substantial factor far greater than the limit predicted by the Rankine-Hugoniot jump conditions, i.e. $B_{\max } / B_{u} \gg 4$. This has been established to be a typical manifestation of high $M_{A}$ shocks, underpinning the importance of kinetic over fluid processes, and has not yet been fully explained.

Periodic signatures in the magnetic field can, of course, be attributed to other factors. Repeated shock crossings modulated by the variability in the upstream dynamic pressure (and/or from the downstream region, in this case the internal pressure of a planet's magnetosphere) do occur and some periodic signature is therefore manifested in the data. These however occur at periods that are irregular, markedly larger than those similar to Figure 2 and a downstream "sheath" signature is typically present. 

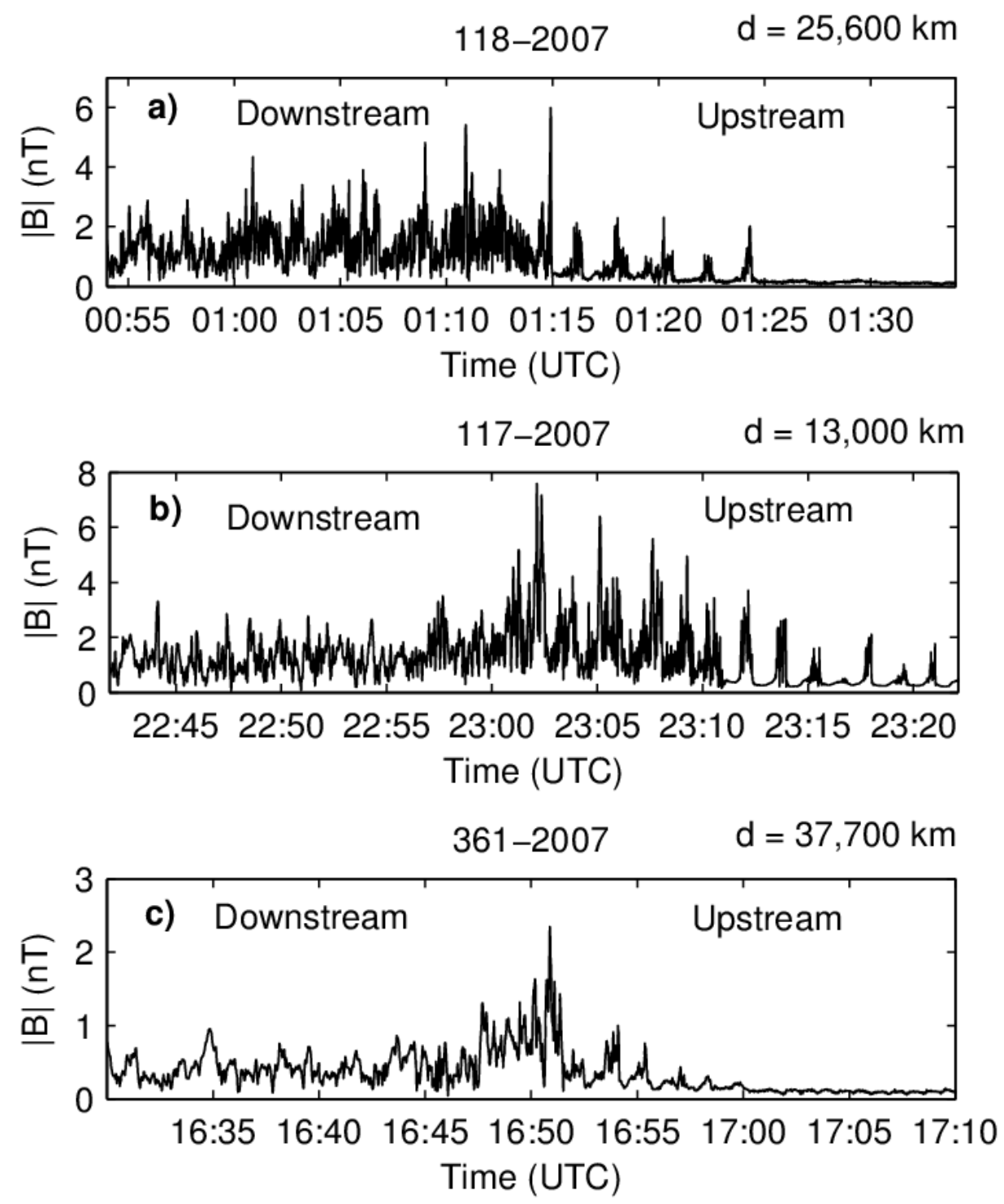

Figure 5.3.: The three panels (a-c) are three examples magnetic field plots of quasi-perpendicular crossings with regular periodic signatures upstream in the highest Mach number regime. These correspond to events 4, 3 and 13 respectively in Figure 5.5 and Table 5.1. The foot thickness, $d$, for each is determined using Equation 5.1 and from knowledge of $\mathrm{V}_{\mathrm{u}}$. Note the different ordinate limits. 


\subsection{Results and Discussion}

Within this regime, we separate the crossings between those that show magnetic field signatures of reformation, given the size and clarity of their quasiperiodic pulses, and those that do not. The criterion is such that there are clearcut 'reformation cycles' from upstream to downstream of the main transition layer similar to those of Figure 5.3. Moreover, for each event that exhibits the upstream features similar to Figure 5.3, we have calculated the period of the cycles (average time between peaks of neighbouring pulses) and find it to be within a narrow range of timescales at which a reformation cycle is predicted to occur. These are shown on Figure 5.4 as being in the range $\sim 0.2-0.3 \tau_{c}$ or $\sim 1.3$ $1.8 \Omega_{c}^{-1}\left(25^{\text {th }}\right.$ and $75^{\text {th }}$ percentiles respectively), where $\tau_{c}\left(=2 \pi \Omega_{c}^{-1}\right)$ is the upstream and undisturbed ion gyroperiod. Table 5.1 lists all the relevant parameters of each event. The average time periods for all crossings are $60-150$ seconds. Looking at one crossing at a time, the peaks of each exhibit very good regularity, i.e. the times between each neighbouring peaks for any given crossing vary by a few seconds. The errors associated with Figure 5.4 are therefore negligible. Since these upstream cycles are temporal variations, it is likely that the number of cycles per crossing varies with shock speed relative to the spacecraft. For example, a spacecraft traversing a fast shock (in the sense of the boundary speed) will spend less time in the foot region and thus will see fewer cycles.

Assuming motion in the upstream field, this range (see Figure 5.4) is consistent with the proton specular reflected turnaround time for the period of magnetic fluctuations. Figure 5.5 shows that, for a range of $\theta_{B n}$, the time it takes for a proton to re-encounter the shock is approximately $0.3 \tau_{c}$ [Schwartz et al., 1983]. We interpret this periodicity to be associated with reformation which 


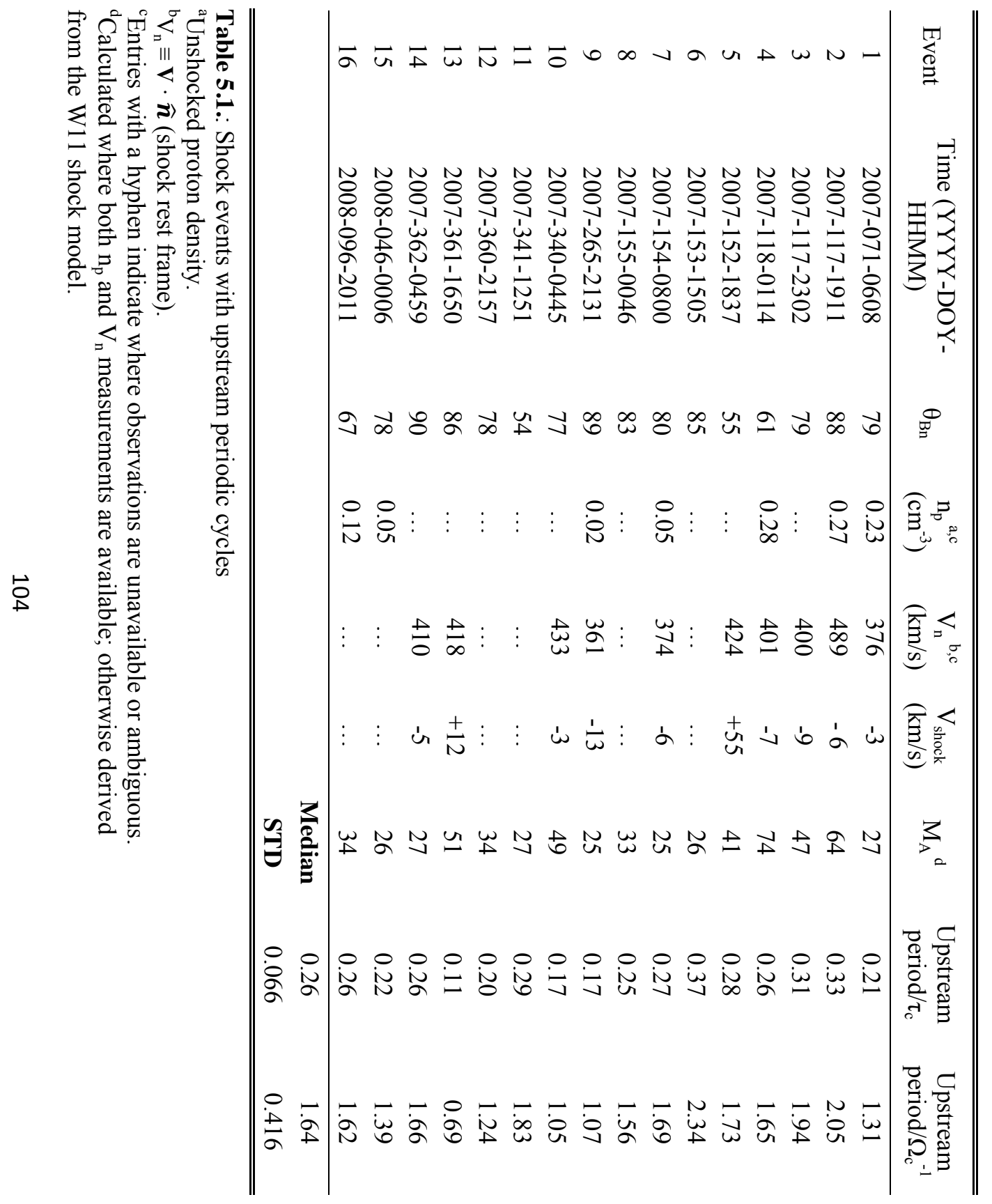




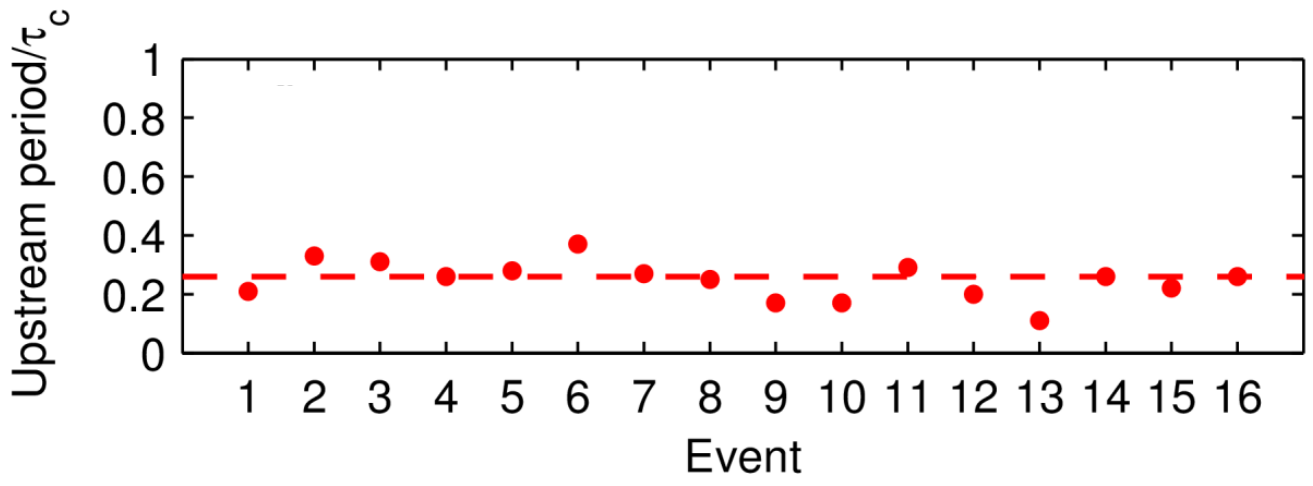

Figure 5.4.: The average period of the upstream cycles for each event (see Table 5.1) normalised to their respective gyroperiods.

involves a periodic modulation of the reflected ions, with the turnaround time of specularly reflected ions being a natural characteristic period of the foot structure. This is supported by hybrid simulations [Yuan et al., 2009] deducing that at high $M_{A}$, shock dynamics appear to be likely dominated by ion reflection. Their results also show that while reformation periods for 1D and 2D results are vastly different at low Mach numbers, they rapidly converge to the ballpark of $0.3 \tau_{c}$ (see Figure 5.5). Earlier work on hybrid simulations also reports quasiperiodic modulation of the reflected with a period just over $\Omega_{c}{ }^{-1}$ and suggests for high $M_{A}$ that ions are reflected dynamically in bunches [Leroy et al., 1982]. More recently, 1-D hybrid simulations running similar plasma parameters to Voyager observations of the Uranian bow shock report localized magnetic field enhancements of a reforming shock occurring at approximately every $1.8 \Omega_{c}{ }^{-1}$ [Tiu et al., 2011].

Other possible sources of a periodic signal may originate upstream, e.g. if there were foreshock or solar wind fluctuations (e.g. Figure 1.10). Foreshock driving is not seen and unlikely due to the quasi-perpendicular configuration of the shock and similarly there are no consistent and/or clear signals in the plasma wave instrument that suggests solar wind fluctuation driving. The observations therefore demonstrate that cyclic reformation is the only plausible process and it is probably controlled by specular reflection. 


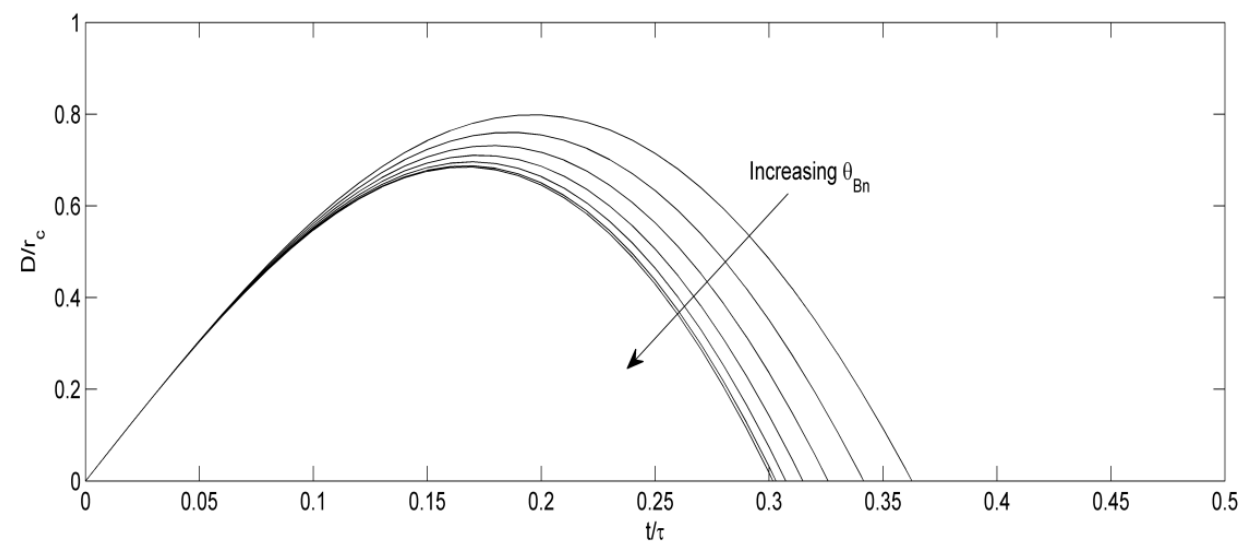

Figure 5.5.: From Equation 5.1. Trajectories of specularly reflected protons from the quasi-perpendicular shock front ( $x$-axis) until first encounter. The displacement $(D)$ is normalised to the convected gyroradius $\left(r_{c}\right)$ and time $(t)$ to the gyroperiod $\left(\tau_{c}\right)$. Each trajectory is of a different $\theta_{B n}$ increasing in the direction of the arrow and ranging from $60^{\circ}$ to $90^{\circ}$ in increments of $5^{\circ}$. Expression derived in Schwartz et al. (1983).

These 54 crossings altogether are highlighted (red or blue) on a parameter space as shown in Figure 5.6a, and we see a significant fraction of crossings in the population revealing this cyclical feature (red). Crossings that were not highlighted (gray) in this regime (above the $80^{\text {th }}$ percentile) were either determined to be quasi-parallel and/or had considerable variability upstream, likely from an active foreshock region and were therefore not included. We have also investigated same-sized populations of the lowest $\left(M_{A}<8\right)$ and middle $(12 \leq$ $\left.M_{A}<17\right)$ ranges to find only one and six crossings with such cyclical feature respectively. We focus on the highest $M_{A}$ regime where the absence or presence of this feature is most clearly distinguishable. Additionally at this regime, we observe multiple upstream peaks over which we can straightforwardly obtain a handle of the periods. The dependence of reformation on $M_{A}$ is clearly corroborated and this also shows that a high $M_{A}$ is a necessary but not sufficient condition. 

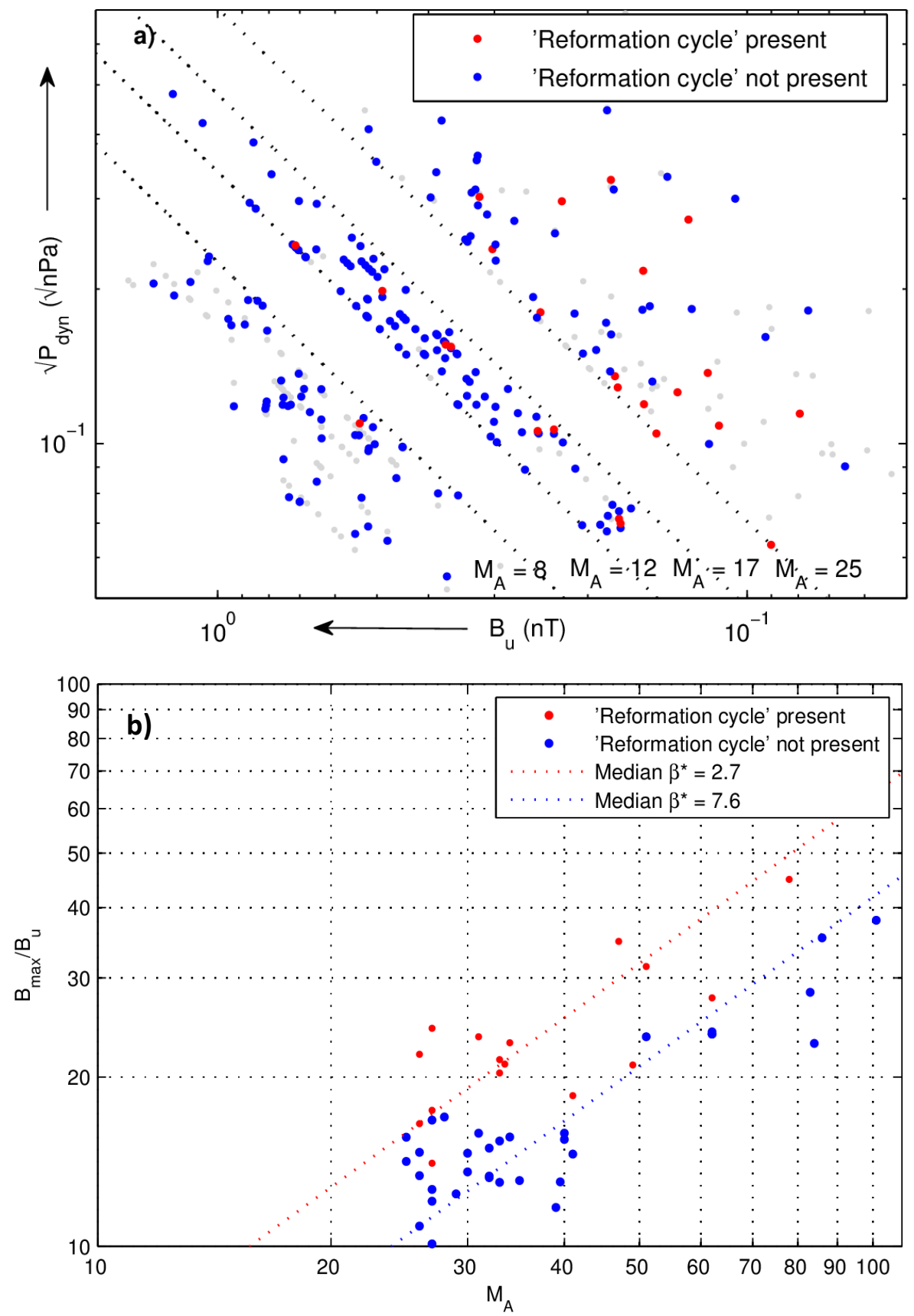

Figure 5.6.: (a) Parameter space of crossings in three regimes of $M_{A}$ highlighting in red the events which exhibit the reformation cycles upstream in contrast with the crossings that do not in blue. The total number of crossings have been divided into three groups of "high" ( $\geq 80^{\text {th }}$ percentile $)$, "medium" $\left(40^{\text {th }}-60^{\text {th }}\right.$ percentiles $)$ and "low" ( $<20^{\text {th }}$ percentile $)$ as marked by the black dashed lines. The crossings that do not lie within any of the three groups have been excluded. (b) New parameter space of the crossings in the highest regime, $\geq 80^{\text {th }}$ percentile or $M_{A} \geq 25$, with $B_{\max }$. The red and blue dashed lines are the medians of the $\beta^{*}$ (as introduced in the text) for the reforming and non-reforming groups respectively. 
The high- $M_{A}$ crossings in Figure 5.6a, however, do not appear to be organized into distinct groups and there is no obvious quantity which separates a reforming from a non-reforming crossing. By taking into account the maximum field $B_{\max }$, Figure 5.6b shows the overshoots $B_{\max } / B_{u}$ versus $M_{A}$ (of each of the highlighted high- $M_{A}$ crossings) revealing a correlation, as expected. The key feature here, on the other hand, is the clear separation between the two groups of crossings and we can infer that shocks undergoing cyclic reformation exhibit a larger overshoot than shocks that do not, for a given $M_{A}$. This suggests that reflected ions may have a role in amplifying the local magnetic field however the causality between these two processes remains unclear.

\subsubsection{An Attempt to Reconcile with $\beta$}

A reasonable next step would be to reconcile the observations with the upstream $\beta$. For instrumentation and spacecraft potential reasons, As highlighted earlier, local upstream ion temperature measurements are very difficult to obtain with Cassini. An alternative approach would be to use the local upstream electron measurements and determine the ion counterpart by some scaling law. However the spacecraft potential washes out the electron distribution in the unshocked solar wind. A proxy $\beta$ is therefore estimated by relating the upstream region, where $B_{u}$ is measured, to the region at which $B_{\max }$ is seen using the momentum flux invariant from the Rankine-Hugoniot equations as follows:

$$
\left[P_{d y n}+\frac{B^{2}}{2 \mu_{0}}+P_{t h}\right]
$$

where $P_{t h}$ is the thermal pressure. The fact that most of the energy upstream resides in the directed bulk flow is employed, i.e.

$$
P_{d y n} \gg \frac{B_{u}^{2}}{2 \mu_{0}}+P_{t h}
$$

At high Mach numbers, the dynamic pressure downstream is reduced to $1 / 4$ of the upstream by taking into account mass conservation and that the compression 
ratio, $\rho_{d} / \rho_{u}$, has a maximum limit of 4 . This is a very good approximation since the compression ratio rapidly increases from a Mach number of unity and reaches its maximum of 4 at relatively low Mach numbers. Combining Equations 5.3 and 5.4 give a relationship between both regions (upstream and location of $B_{\max }$ ) as

$$
P_{d y n} \approx \frac{1}{4} P_{d y n}+\frac{B_{\max }^{2}}{2 \mu_{0}}\left(1+\beta^{*}\right)
$$

Combining Equations 4.8 and 4.4 gives an equation for the ratio of the thermal to magnetic pressures, $\beta^{*}$, at the location of $B_{\max }$

$$
\beta^{*} \approx \frac{3}{2}\left(M_{A} \frac{B_{u}}{B_{\max }}\right)^{2}-1
$$

The $\beta^{*}$ is calculated for each of the highlighted crossings. The median $\beta^{*}$ for each group of crossings is plotted in Figure $5.6 \mathrm{~b}$ and it can be seen that the median $\beta^{*}$ is considerably less for the crossings that show the reformation feature upstream across the $M_{A}$ range. This technique at least qualitatively separates the crossings into a low and high $\beta$ regime, whether or not the values of $\beta^{*}$ is directly representative of the upstream ion $\beta$, believed to be one of the main control parameters for reformation. This quantitative relationship, or lack of, between $\beta^{*}$ and the upstream $\beta$ can be tested at Earth with the availability of continual and simultaneous magnetic field, density and temperature measurements across a shock.

The role of the upstream $\beta$ in influencing the outcome of reformation has been widely advocated by simulators [e.g. Morse et al., 1972; Hellinger et al., 2002]. Although not yet experimentally verified, the current understanding is that reformation is supressed at sufficiently high $\beta$. As introduced in $\$ 1.9 .3$ and shown in Figure 5.7, the reflected ion population creates a hole in the velocityspace distribution. This hole leads to a depression in the magnetic field strength relative to the surrounding ring-vortex thus resulting to the formation of a new ramp with time. The shock eventually reforms as the new ramp progresses. At 
high enough upstream ion $\beta$, the temperature and thus the thermal ion velocity is sufficient to smear out and plug the hole between the incoming and reflected populations.

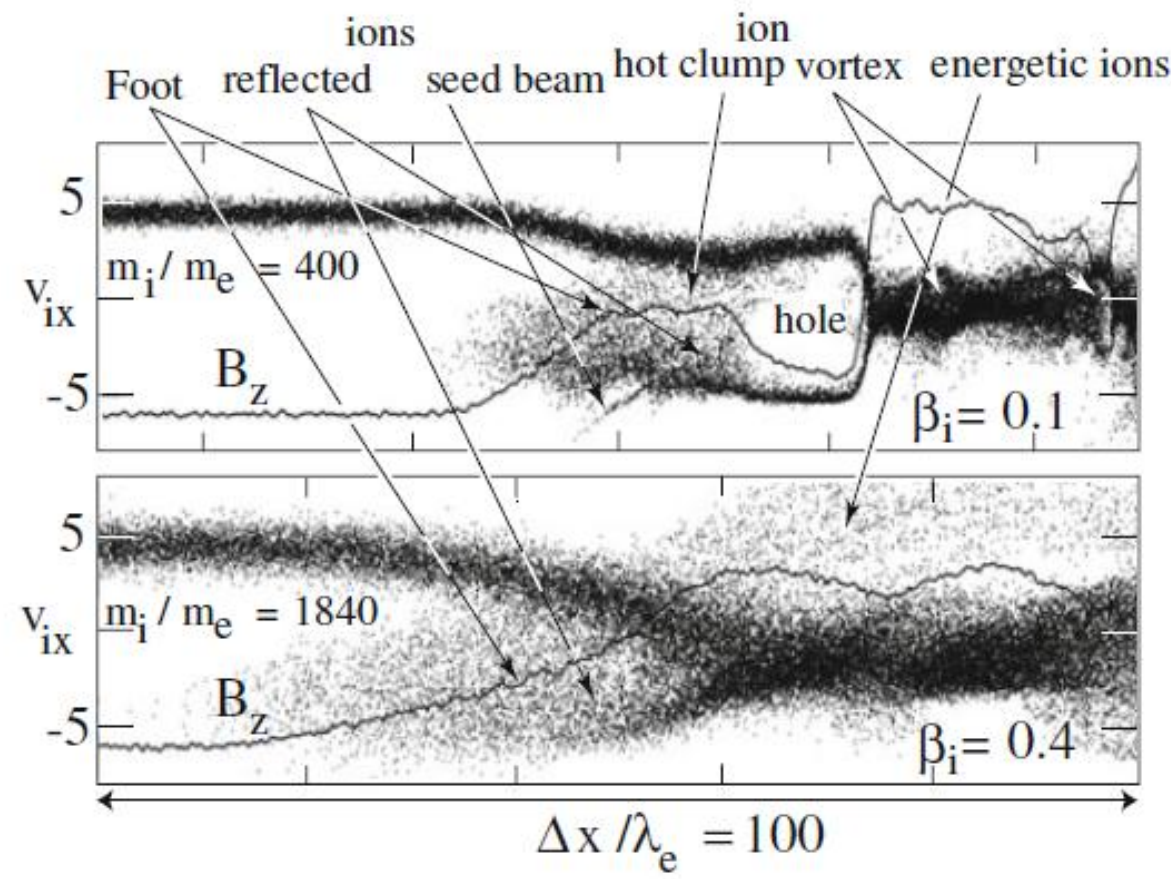

Figure 5.7.: Ion velocity-space distributions for $\beta_{i}=0.1$ (top) and $\beta_{i}=0.4$ (bottom) [Scholer et al., 2003]. A hole is created between a reflected ion beam and incoming population which depresses the magnetic field relative to the surrounding ring-vortex and leads to the formation of a new ramp. This hole is smeared out at a high $\beta_{i}$ and thereby supressing reformation.

\subsection{Conclusion}

Indeed, attempting to dissect a single shock crossing here to uncover its microphysics is not likely to be sufficiently instructive; especially in the prevalent analyses with limited particle data sets. This work nonetheless provides a picture of a quasi-perpendicular shock wave's magnetic character at the underexplored high $M_{A}$ and the observations reveal the prominent role of specular reflection in controlling cyclic reformation. Another finding here is the connection between reformation and magnetic field amplification with such shocks having a distinctly higher overshoot. Hybrid simulations would help shed 
light on this and overall this work can complement ongoing theoretical work and simulations. We anticipate our study to provide a deeper insight into collisionless shocks, particularly in astrophysical regimes where they are central to both the structure and dynamics of supernovae. 


\section{The Magnetic Structure of Saturn's Magnetosheath}

\subsection{Introduction}

The magnetosheath of a planet is the region between the freestream solar wind and the planetary magnetosphere. It is bounded by the bow shock, which deflects and slows down the solar wind, and the magnetopause obstacle, which the flow diverts around and the convected magnetic field lines overall drape tangentially to. The region is therefore an important site for both plasma micro- and macro-processes such as turbulence, instabilities, magnetic reconnection and plasma depletion layers (PDL - close packing of magnetic field lines near the magnetopause surface) [Lepping et al., 1981; Russell, 1976; Violante et al., 1995; Zwan and Wolf, 1976]. Micro-processes such as reconnection occur when the diffusive term becomes dominant over the convective term of the magnetic Reynolds number i.e. $\eta>U L$ (where $\eta$ is the magnetic diffusivity, $U$ the flow velocity and $L$ the characteristic length of the plasma structure). This breaks down the frozen-in flux condition which states that the magnetic field lines are 'frozen' into and move with the plasma fluid.

The supersonic solar wind is rapidly slowed down and heated into a subsonic regime immediately downstream of the shock, with the effect greatest at the subsolar region where the shock front is normal to the flow. Analogous to the diverging segment of a 'de Laval' nozzle, the magnetosheath cross-sectional area increases with solar zenith angle. The flow is then further driven by the associated tension in the draped IMF in addition to the substantially lower back pressure at the terminator region far downstream. This accelerates the flow as it diverts around the magnetosphere and continues to do so until it is supersonic and the 
freestream solar wind conditions are eventually met well downstream of the terminator.

Studies of the magnetosheath merge important subject areas of both collisionless shocks and magnetospheric physics. Its field and particle conditions are both an end result, to characterise the nature of a bow shock, and a prerequisite, to understand magnetospheric dynamics via mass, energy and momentum transfers from the solar wind to planetary magnetospheres. Draping of magnetic field lines is one of the processes canonically understood to influence magnetic reconnection, and thus also the extent of the magnetosheath plasma depletion layer [Dungey, 1961; Sonnerup, 1974; Slavin et al., 1983]. This is a condition widely accepted for the terrestrial magnetopause. Further studies corroborate this importance on reconnection onset at other planets such as at Mercury [Slavin et al., 2009] and more recently DiBraccio et al. [2013] showed that reconnection occurs for a wide range of magnetic shear angles, the rotation of the magnetic field from the magnetosheath into the magnetosphere, likely because of the low $\beta$ conditions. Gershman et al. (2013) also reported a large-scale PDL often forming just exterior of Mercury's subsolar magnetopause as a result of piled-up draped magnetic flux around the magnetosphere. This has been attributed to the low average upstream $M_{A}$. Masters et al. (2014), on the other hand, show no PDL response to cross-magnetopause magnetic shear because the magnetic flux transport rates associated with reconnection are too low to have any effect. Nonetheless, it is expected that the IMF orientation strongly controls where reconnection is occurring because of the $\beta$-imposed constraint of close to anti-parallel fields required for reconnection onset [Masters et al., 2012].

One of the earliest studies of the magnetosheath predicted the draping of the magnetic field using a gas-dynamic model [Spreiter et al., 1966; Spreiter and Stahara, 1980]. This was achieved using the hydrodynamics of a single-fluid, nondissipative gas to describe the bulk flow around a planet with a prescribed non-self-consistent magnetic field convecting in unison. Further work was carried out by Fairfield [1969] and Crooker et al. [1985] where they compared this model to Earth observations of draping in the dayside magnetosheath. The latter used a multi-spacecraft technique with ISEE 3 upstream measuring the IMF as 
inputs to the gas-dynamic model and compared with time-lagged observations by ISEE 1 in the magnetosheath. They concluded that the observed draping near the dayside magnetopause is relatively consistent with the simple gas-dynamic model.

The magnetosheath of Saturn has commonalities to that of the Earth and Jupiter but also a significant uniqueness [Richardson, 2002; Sergis et al., 2013]. It is distinctive in its (dayside) geometry which is governed by the competing anti-planetward pressures, due to internal magnetospheric processes [Achilleos et al., 2008], impinging on the magnetopause and the dynamic pressure of the much more tenuous solar wind plasma upstream of the bow shock. Two main features of its global geometry are the subsolar thickness, dictated by both internal and external pressure variability, and non-axisymmetry of the magnetopause by polar flattening; both of which are expected to have an effect on the magnetosheath's structure. An analytical treatment has been developed using ideal MHD to describe the conditions in the magnetosheath of a non-axisymmetric magnetopause [Erkaev et al., 1996; Farrugia et al., 1998]. The IMF orientation was found to play an important role in controlling the properties of the magnetosheath as a consequence of the deviation from axisymmetry. The magnetic field in the magnetosheath was shown to compress with the effect most pronounced nearer the magnetopause. The field lines were shown to rotate towards the planet's rotation axis, an additional effect only present in a non-axisymmetric case. This is shown in Figure 6.1 from Desroche et al. (2012) for an upstream IMF with a clock angle of $85^{\circ}$ (i.e. clockwise from north). This was also found to have a significant influence on the plasma flow, with the acceleration triggered by the magnetic tension force in a direction perpendicular to the magnetic field lines [Erkaev et al., 2011, 2012]. Saturn's magnetosheath is also physically different with a high plasma $\beta$ (ratio of particle to magnetic field pressures) environment [Masters et al., 2012] owing to the high Alfvén Mach number bow shock. Both the Mach number and magnetosheath plasma $\beta$ increase monotonically with heliocentric distance. 


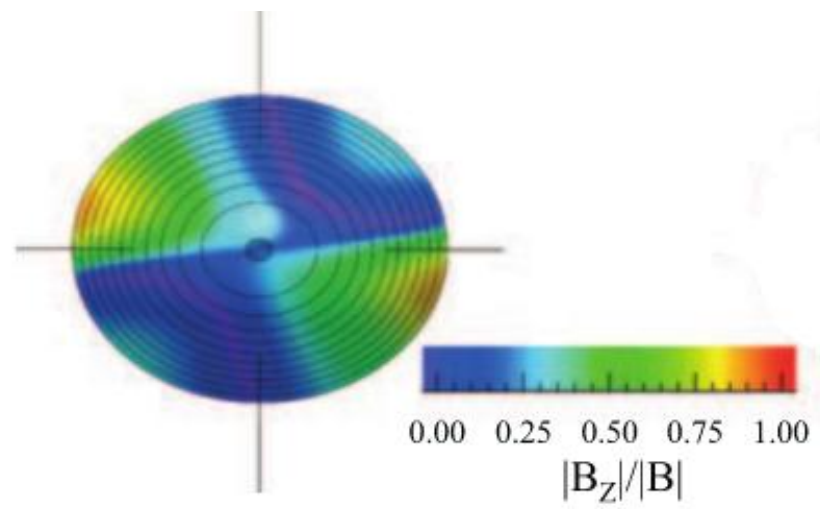

Figure 6.1.: $Z$ (north-south) component of the magnetic field just exterior of the magnetopause normalised to the magnitude. The IMF clock angle is $85^{\circ}$ (near equatorial) and shown to rotate towards the $Z$ axis as the IMF is convected through the mgnetosheath. This effect is unique to non-axisymmetric obstacles. In this study by Desroche et al. (2012), the square of the ratio of equatorial to polar axes is 1.5 . Licence \#3790360790635.

In this chapter, we investigate the large-scale overall configuration of Saturn's magnetosheath magnetic field using observations made by the Cassini spacecraft. While ongoing studies of high-latitude Cassini orbits aim to constrain the extent of magnetospheric polar flattening, here we present the magnetic field structure of the magnetosheath which is largely at lower latitudes. We compare and contrast the magnetic field observations with outputs from the BATSRUS MHD model in each of the equatorial and meridional planes and further compare four cases when the IMF orientation was relatively steady while Cassini traversed the magnetosheath with an analytical model describing draping between axisymmetric boundaries.

\subsection{Observations}

\subsubsection{Data Selection}

In this section, we introduce the type of data selected, the applicability and limitations of models used, and the approaches to conduct the analyses. We use data obtained from Cassini's onboard fluxgate magnetometer (MAG) [Dougherty et al., 2004] from which boundary crossings and magnetosheath signatures are identified. Since we are interested in the large-scale spatially-dependent structure of the magnetosheath, we have selected 106 complete and uninterrupted magne- 
tosheath traversals from Saturn Orbit Insertion (SOI) in 2004 to 2010 inclusive. These are both inbound (bow shock to magnetopause) and outbound (magnetopause to bow shock) and exclude excursions due to global boundary oscillations or surface waves. Such excursions are generally identified as a series of crossing over a timescale much shorter than the magnetosheath traversal [Mistry et al., 2014].

The coordinate system used throughout this study is the Cartesian Kronocentric Solar Magnetospheric (KSM) system. Figure 6.2 highlights the spacecraft position relative to Saturn where the magnetosheath is observed. The total coverage sums up to 2,486 hours with $84 \%$ of this on the dayside, $65 \%$ and $35 \%$ on the dawn $(<1200$ LT) and dusk ( $>1200$ LT) flanks respectively, and particularly limited to lower and equatorial latitudes. The magnetic field and position measurements used are at 1-min resolution. This is of sufficient resolution and a higher time resolution does not improve the analysis since, given the range of time over which the analysis is conducted, the adjacent samples are not likely to be statistically independent. The angles are measured as the meridional angle which has a range of $-90^{\circ}$ (southward) to $+90^{\circ}$ (northward) and the azimuthal angle 

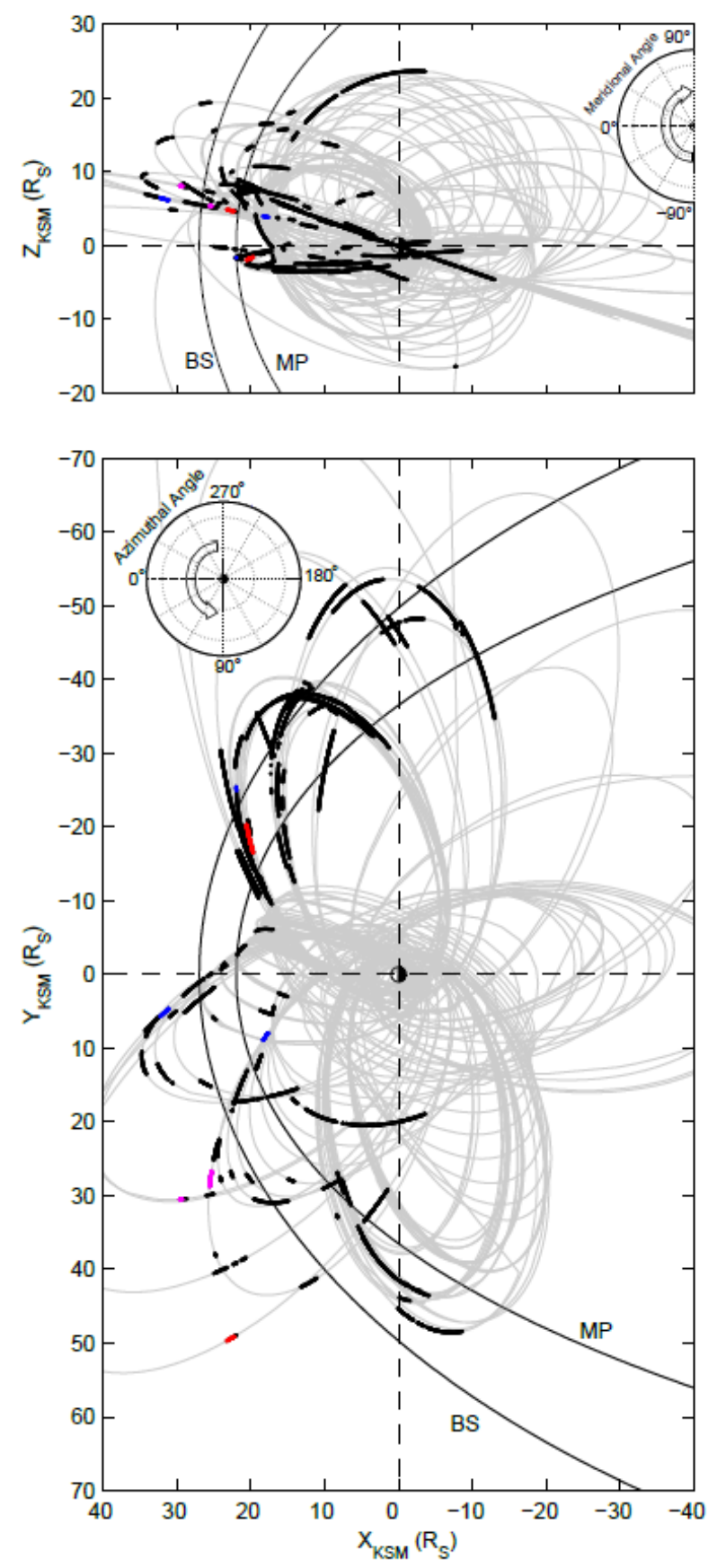

Figure 6.2.: An overview of the trajectory of the Cassini spacecraft (grey) between July 2004 - December 2010 with observed magnetosheath boundary-to-boundary traversals (black) indicated and projected onto (a) the $X-Z$ and (b) the $X-Y$ planes. The IMF orientation was relatively steady throughout the traversals in colour. Blue indicates northward IMF, red indicates traversals used in the case study and magenta indicates a combination of both. In both figures the projections of the Kanani et al. [2010] magnetopause and Went et al. [2011] bow shock models are shown with median subsolar distances of $22 R_{S}$ and $27 R_{S}$ respectively for a solar wind dynamic pressure of $\sim 0.02 \mathrm{nPa}$. The medians of the sets of respective boundary crossings are used so that errors in the models or extreme events which produce anomalous estimates do not significantly skew the determination of the typical subsolar distance. The insets on the top and bottom panels define the meridional and azimuthal angles respectively. 
which is calculated counter-clockwise from $+X$ and has a range $0^{\circ}$ to $360^{\circ}$ (see insets in Figure 6.2).

\subsubsection{Upstream Conditions and the Overall Configuration of the Magnetic Field in Saturn's Magnetosheath}

Cassini is presently the only spacecraft probing the kronian vicinity. This poses a difficulty in magnetosheath analyses since in-situ measurements of upstream conditions, which play an important role in driving the magnetosheath structure and dynamics, cannot be obtained simultaneously. In most cases, the timescales of solar wind variability are small compared to Cassini's time of flight through the magnetosheath, meaning the spacecraft is measuring particle and field parameters influenced by upstream conditions different to those measured just before the spacecraft crossed the bow shock (say for an inbound flight path). The bow shock and magnetopause boundaries exhibit global oscillations principally driven by the variability in the solar wind dynamic pressure resulting in changes to the location of the magnetosheath relative to Saturn (further away at lower dynamic pressures and vice versa). In addition, the two boundaries may respond differently to the solar wind dynamic pressure [Slavin et al., 1985; Hendricks et al., 2005] hence a change in the dynamic pressure is not necessarily proportional to the magnetosheath subsolar and polar thicknesses i.e. the planetocentric distances between the magnetopause and the bow shock at $\mathrm{X}_{\mathrm{KSM}}=0$ and $\mathrm{Z}_{\mathrm{KSM}}=0$ respectively (neglecting aberration).

Cassini sampled the upstream environment of Saturn for over a year before Saturn Orbit Insertion and the IMF orientation was measured to exhibit a bimodal distribution of the Parker spiral (in the ecliptic plane) angle averaging $90.6^{\circ} \pm$ $0.4^{\circ}$ and $276.4^{\circ} \pm 0.5^{\circ}$ (for angles $<180^{\circ}$ and $\geq 180^{\circ}$ respectively) with a slight meridional (out of the ecliptic plane) angle averaging $1.4^{\circ} \pm 0.3^{\circ}$ [Jackman et al., 2008]. 

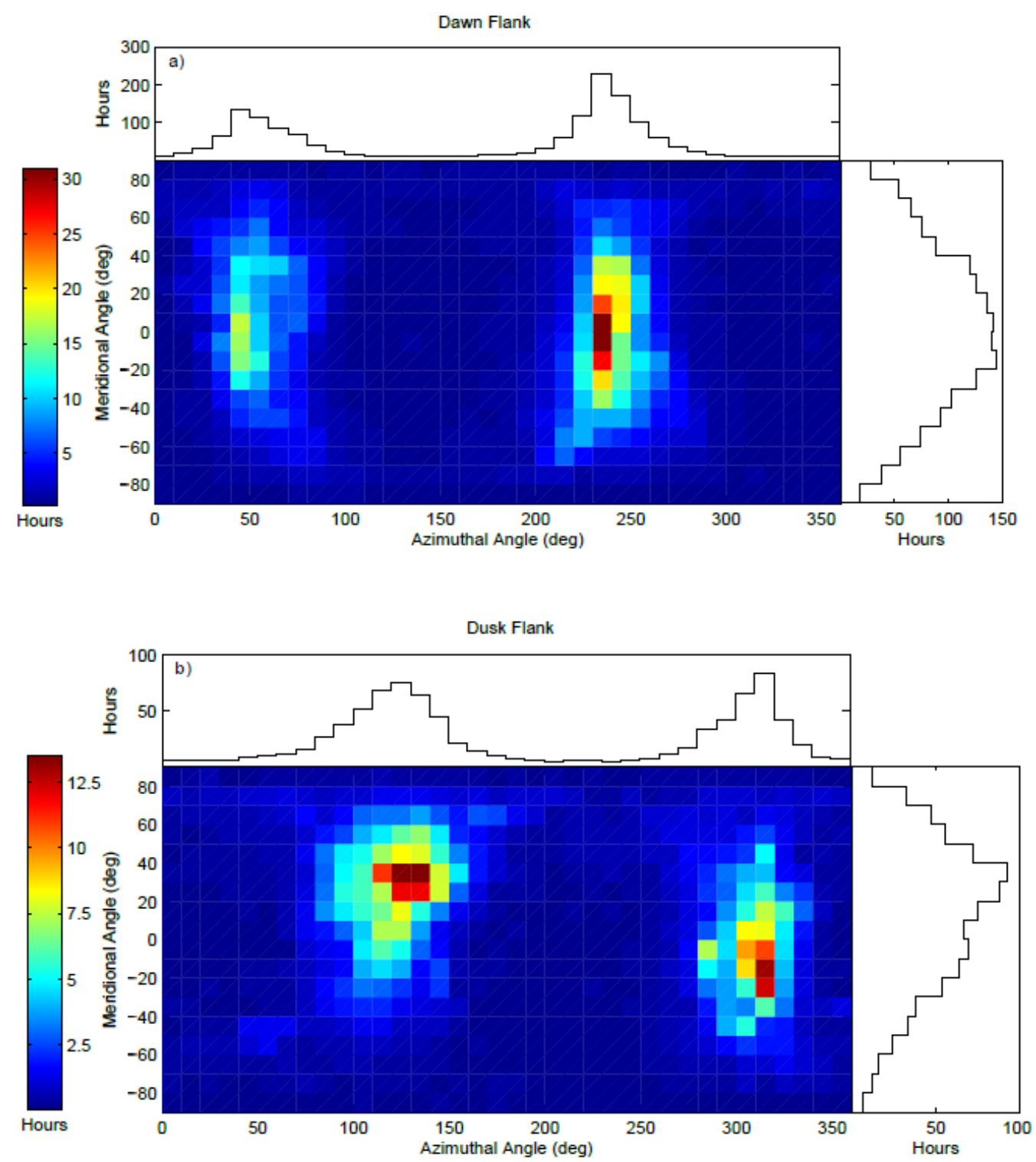

Figure 6.3.: The statistical configuration of the magnetic field on the a) dawn flank and b) dusk flank of the magnetosheath. The 2-D histograms are colour scaled to the length of time the magnetic field has been observed in a particular combination of meridional and azimuthal directions. The adjacent histograms project these angles individually. 
Figure 6.3 illustrates the distribution of the observed configuration of the magnetic field in the magnetosheath for both the dawn and dusk flanks. The bin size for all plots is $10^{\circ}$. The colour plots are $2 \mathrm{D}$ histograms consolidating both meridional and azimuthal angles. The colour bar represents the length of time the angles were observed to be in a particular orientation. The histograms on their sides project the distributions of the individual angles. The orientation in the dawn flank shows a bimodal distribution of the azimuthal angle, with peaks shifted to the left from the Parker spiral, averaging at $65.9^{\circ} \pm 0.2^{\circ}$ and $245.5^{\circ} \pm$ $0.1^{\circ}$ (for angles $<180^{\circ}$ and $\geq 180^{\circ}$ respectively). The dusk flank has peaks shifted to the right from the Parker spiral and averaging at $112.9^{\circ} \pm 0.2^{\circ}$ and $297.4^{\circ} \pm$ $0.2^{\circ}$. The relative amplitude of each pair of peaks indicate the ratio of time Cassini spent on the inward and outward pointing regions either side of the Heliospheric Current Sheet. The meridional angles average at $1.9^{\circ} \pm 0.1^{\circ}$ for the dawn flank and a more substantial $12.1^{\circ} \pm 0.2^{\circ}$ for the dusk flank. The distributions are also more dispersed than that of the IMF upstream of Saturn [Jackman et al., 2008].

\subsection{Results}

\subsubsection{MHD Simulations}

We compare the magnetic field observations to the global BATSRUS MHD model which solves the governing MHD equations using a conservative finite-volume method [Gombosi et al., 2002; Jia et al., 2012]. The model has been tailored to simulate the kronian environment and outputs are generated for two IMF limiting cases: duskward and northward. This will allow us to estimate the (predominantly dayside) angular change of the magnetic field with respect to longitude on the equatorial $X-Y$ plane (for duskward IMF) and the angular change with respect to latitude on the meridional $X$ - $Z$ plane (for northward IMF). We do not place particular emphasis on the directions of the IMF, but rather on their alignments with the planes such that the third components orthogonal to the two planes are zero. These results are compared against observations to assess how well the MHD simulations capture and thus predict field line draping and to reveal any asymmetry between the two planes. The expectation is that polar- 

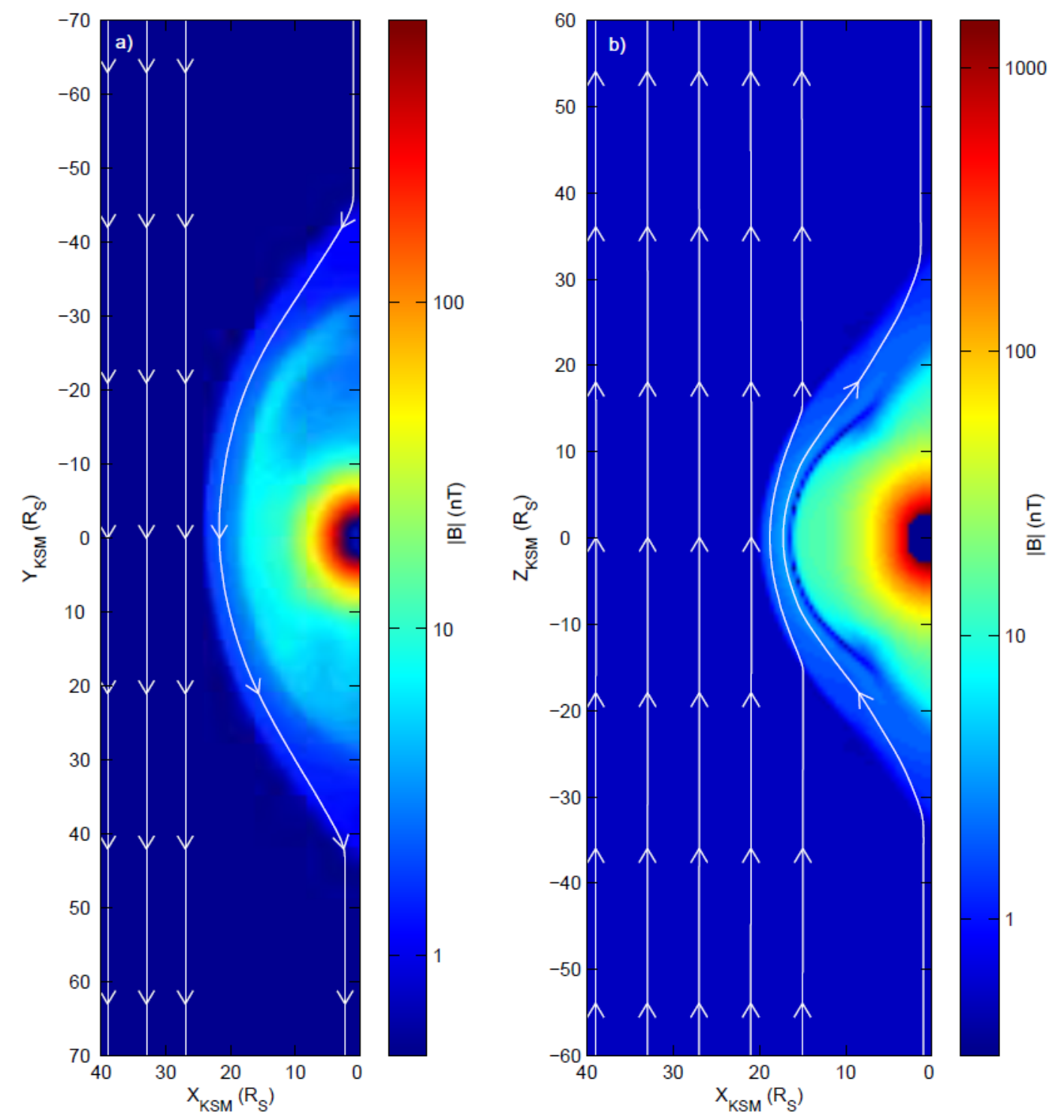

Figure 6.4.: BATSRUS MHD simulation snapshots of dayside draping of the magnetic field for a) duskward and b) northward IMF orientations along the $X-Y\left(Z_{K S M}=0\right)$ and $X-Z\left(Y_{K S M}=0\right)$ planes respectively. The colour scales represent the logarithmic magnetic field magnitude and the white arrows are magnetic field lines which bend upon encountering the bow shock (first anti-planetward boundary) and arrange tangentially to the magnetopause (boundary enclosing high magnetic field magnitude region). See Jia et al. (2012) for a comprehensive report on the simulations boundary conditions and output interpretations. 
flattening of the magnetosphere is manifested on the draping pattern of the field lines and hence that the asymmetry can therefore be estimated.

\begin{tabular}{cccccc}
\hline Direction & $\mathrm{B}_{\mathrm{x}}(\mathrm{nT})$ & $\mathrm{B}_{\mathrm{y}}(\mathrm{nT})$ & $\mathrm{B}_{\mathrm{z}}(\mathrm{nT})$ & $\mathrm{D}_{\mathrm{p}}(\mathrm{nPa})$ & Grid Resolution $\left(\mathrm{R}_{\mathrm{s}}\right)$ \\
\hline Northward & $-2.41 \mathrm{E}-05$ & $-1.28 \mathrm{E}-05$ & $4.96 \mathrm{E}-01$ & $4.22 \mathrm{E}-04$ & 0.5 \\
Duskward & $3.38 \mathrm{E}-05$ & $4.94 \mathrm{E}-01$ & $3.26 \mathrm{E}-05$ & $2.43 \mathrm{E}-04$ & 0.5 \\
\hline
\end{tabular}

Table 6.1.: Upstream conditions of MHD simulations

Figure 6.4 is a snapshot of the two MHD simulated IMF configurations of duskward and northward viewed at $Z_{\mathrm{KSM}}=0$ and $\mathrm{Y}_{\mathrm{KSM}}=0$ respectively where the magnetic field vectors are perfectly aligned with the planes. The precise location and shape of the magnetopause are ambiguous but the draping pattern of the field lines and hence their angular change with longitude and latitude can be clearly deduced. The upstream conditions of both runs are given in Table 1 and are set such that the IMF is initially northward and duskward respectively.

Figure 6.5 shows the distribution of the observed azimuthal angles against local time. There are two clear linear correlations showing organised draping in the equatorial plane. Near to the subsolar point ( 1200 LT), the corresponding angles are $\sim 90^{\circ}$ (duskward) and $\sim 270^{\circ}$ (dawnward), indicative of the configuration at which the IMF is incident on the Saturnian magnetosphere and consistent with the expected directions of the Parker spiral. The gradient in the meridional angle with respect to local time for both duskward and dawnward IMF orientations are very similar and there is no indication of dependence on direction on a global scale. With increasing (decreasing) local time, the azimuthal angle of the magnetic field lines increases (decreases), asymptotically approaching the planar geometry of the magnetopause. The red line is the MHD simulated draping of a magnetic field line for a duskward configuration in the magnetosheath (see Figure 6.4a) taken at $Z_{\mathrm{KSM}}=0 \mathrm{R}_{\mathrm{S}}$. The angles deduced are averages at different local times in the magnetosheath proper. The MHD model is in good agreement with the observations and reveals a gradient of 0.47 degrees of azimuthal angle per degree longitude for $Z_{\mathrm{KSM}}=0 \mathrm{R}_{\mathrm{S}}$. 


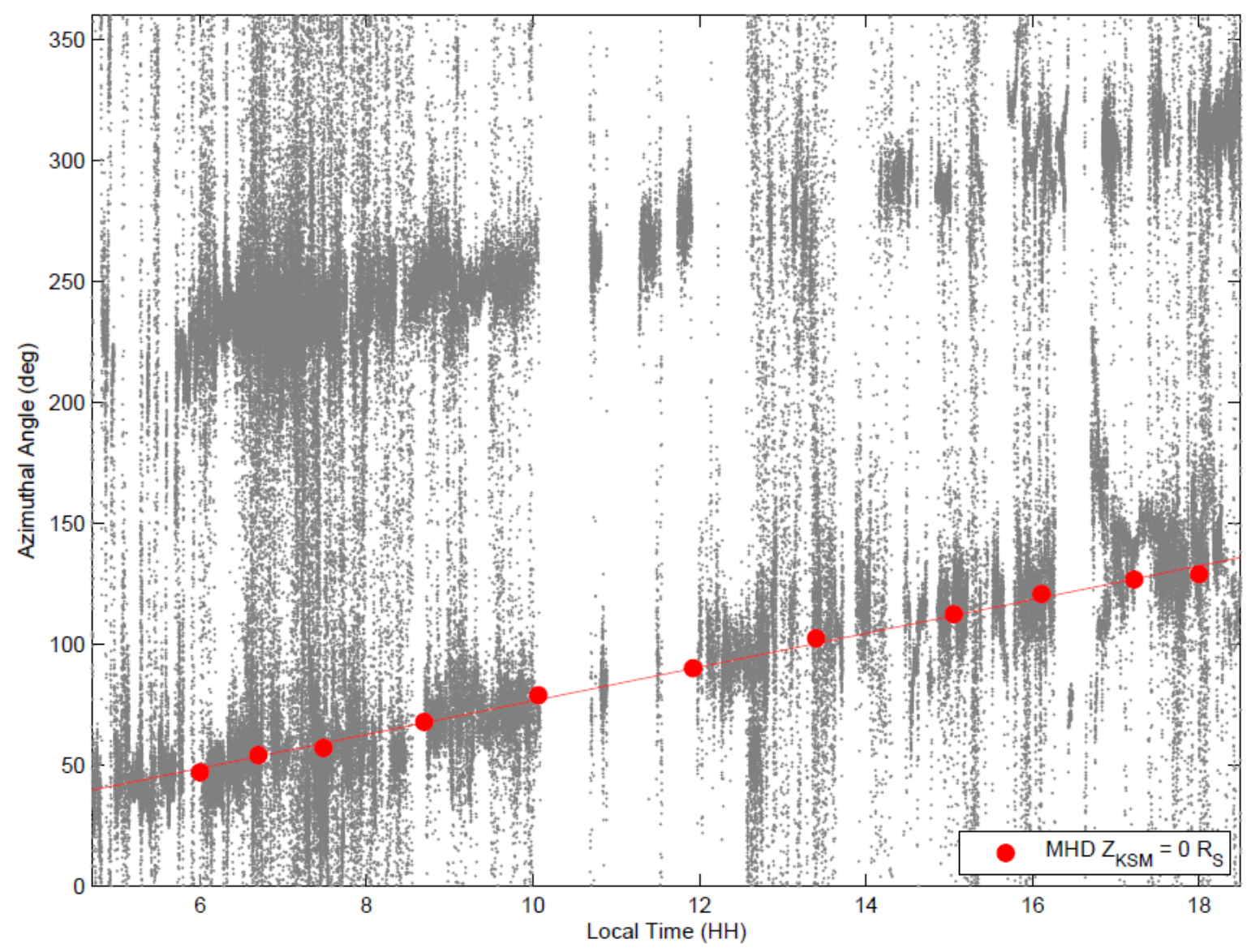

Figure 6.5.: Distribution of the observed azimuthal angle in the magnetosheath with local time projected on a plane. Overlain are the MHDsimulated angles at different local times for a duskward IMF. 


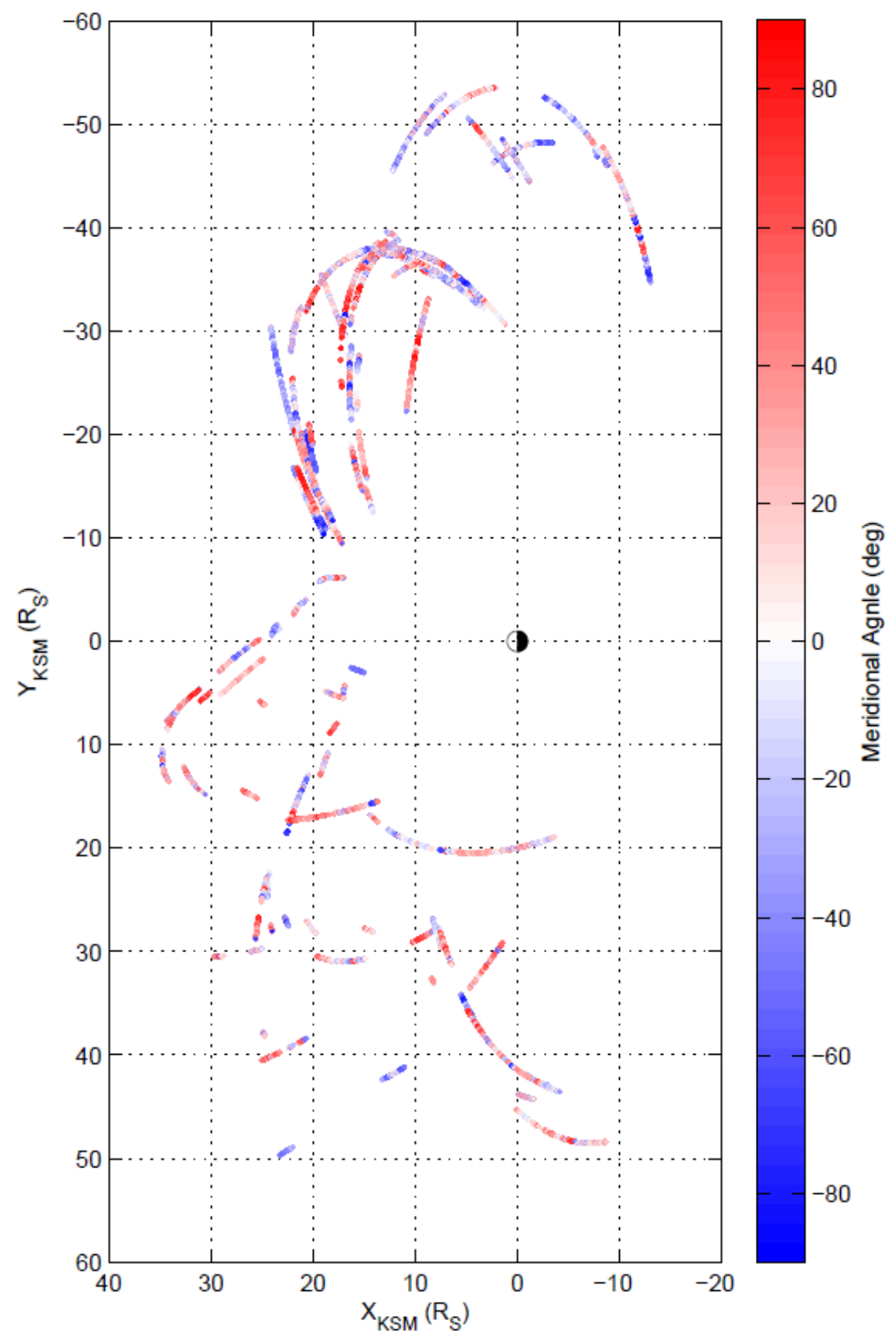

Figure 6.6.: Distribution of observed meridional angle on the equatorial plane. 
Quantifying the angular change per degree latitude on magnetic field lines in the meridional plane is more difficult since the range of latitudinal coverage is far more restricted than that of the longitudinal coverage. In addition, the IMF is statistically most likely to be incident at the Parker spiral angle which lies in the $X-Y$ plane making it impossible to assess the latitudinal draping as simulated in Figure 6.4b. The gradient from this MHD plot reveals a draping of 0.54 degrees per degree latitude for $\mathrm{Y}_{\mathrm{KSM}}=0 \mathrm{R}_{\mathrm{S}}$.

What could be tested, however, was the distribution of the meridional angle on the equatorial plane as shown in Figure 6.6. The purpose of this is to show the significance of a $Z$-component in the magnetosheath field. Since the unshocked IMF generally lies in the equatorial plane, we would expect the field in the magnetosheath to also remain in that plane. Figure 6.6 , on the other hand, shows significant Z-component in the field which may be attributed to the nonaxisymmetric magnetosheath as advocated by Erkaev et al. (1996) and this is further interpreted in $\$ 6.4$.

\subsubsection{Correspondence between observations and predictive model}

Kobel and Flückiger [1994] developed an analytical model (herein referred to as KF94) to characterize the magnetic field in the magnetosheath which has been used extensively in Earth studies [e.g. Longmore et al., 2006; Cooling et al., 2001; Petrinec, 2012] as well as at Mercury and Saturn. The model describes a static magnetic field in the magnetosheath by means of a scalar potential which is a solution to Laplace's equation. The model uses the IMF as input, imposes jump conditions at the first boundary (bow shock) and a boundary condition is set such that the magnetic field is zero at the second boundary (magnetopause). Two key features of this model are that the boundaries are paraboloids symmetric along the planet-Sun line and field-flow coupling effects are not taken into account. 
A formal procedure to carry out a comparison of the observed magnetosheath magnetic field with the model and hence testing the model's ability to predict the draping pattern is best done via a statistical study (such as in Longmore et al. [2006]). This work used a multi-spacecraft technique with the ACE spacecraft providing the IMF data while the Cluster spacecraft provided good coverage of the magnetosheath. The effects of boundary motion were overcome by normalizing each measurement within the magnetosheath to a local position between the two boundaries. As for the effects of solar wind variability, the magnetic field measurements in the magnetosheath were normalized to corresponding time-lagged ACE measurements. Since the standoff distance of a boundary is determined only at the time of crossing, it is not possible to determine the locations of both boundaries as well as the upstream conditions simultaneously at any given time using a single spacecraft such as we have with Cassini at Saturn. In order to mitigate transient effects, a case study approach is taken using four time series when the IMF orientation is relatively steady throughout Cassini's inbound traversals in the magnetosheath. The model is employed to predict the draping pattern of the field lines in the magnetosheath using the IMF as measured by Cassini before crossing the bow shock. The clock angles of the predicted field lines will then be compared with observations by Cassini during its magnetosheath traversals. An instructive comparison would be of the magnetic field against maps of the near-magnetopause region by Desroche et al. [2013] and/or profiles showing how the magnetic field evolves as a consequence of nonaxisymmetry along the subsolar line in the magnetosheasth [Erkaev et al., 1996; Farrugia et al., 1998]. The aforementioned limitations, however, do not allow for the variability to be separated from being spatial or temporal even for the steadiest traversals. The standard deviation of the magnetic field along the magnetosheath was found to be up to $15^{\circ}$ for steady cases and thus cannot distinguish a near-magnetopause magnetic field orientation from an adjacent one.

In this section we conduct a case study to examine the correspondence between the observed draping in the magnetosheath with that predicted by KF94 under different IMF orientations. By testing its validity, this will also provide additional means in approximating the magnetic field in a region where Cassini is 
not present to make observations. The magnetosheath during these four traversals was measured to have relatively steady IMF orientations throughout the traversal, also used in the work by Masters et al. [2014]. This one-to-one approach is not suitable with the MHD simulation since the grid size near the magnetopause and bow shock is $\sim 0.5 \mathrm{R}_{\mathrm{s}}$. Typically a traversal can span six grids and this would thereby return six vectors which is not useful for this study considering the high variability of the magnetic field in the magnetosheath. The inset in Figure $6.7 \mathrm{c}$ shows the four different clock angles considered. They are categorized into a higher $B_{z}$ component, labelled $\mathrm{S}$ and $\mathrm{N}$ for southward and northward orientations respectively, and a lower $B_{z}$ component labelled $\mathrm{O} 1$ and $\mathrm{O} 2$. Figures $6.7 \mathrm{a}$ and $6.7 \mathrm{~b}$ show the pairs of observed (red) and KF94 predicted (black) 3D vector plots for the $\mathrm{S}$ and $\mathrm{O} 1$ orientations during their traversals respectively.

These traversals started and ended at $\sim 5 \%$ of the transit time from each boundary to alleviate the effects of near-boundary activities (such as the PDL near the magnetopause) which are not accommodated by KF94. The four points corresponding to every time history do not represent the same fractional distance since each traversal had a different transit time. Since boundaries can only be observed during the time of the crossing, determining an accurate fractional distance in the magnetosheath during a traversal is not possible. However, full traversals such as these in the case study are generally caused by magnetospheric compression/expansion and we therefore expect to traverse the magnetosheath monotonically from bow shock to magnetopause or vice versa. IMF orientations with higher $B_{z}(\mathrm{~S}$ and $\mathrm{N})$ show a significant clock angular rotation between the observations and KF94. This rotation becomes less pronounced with increasing equatorial orientation (O2 then $\mathrm{O} 1)$. In addition, the rotation tends to increase nearer the magnetopause for $\mathrm{S}, \mathrm{N}$ and $\mathrm{O} 2$ and there is no correlation for $\mathrm{O} 1$. 

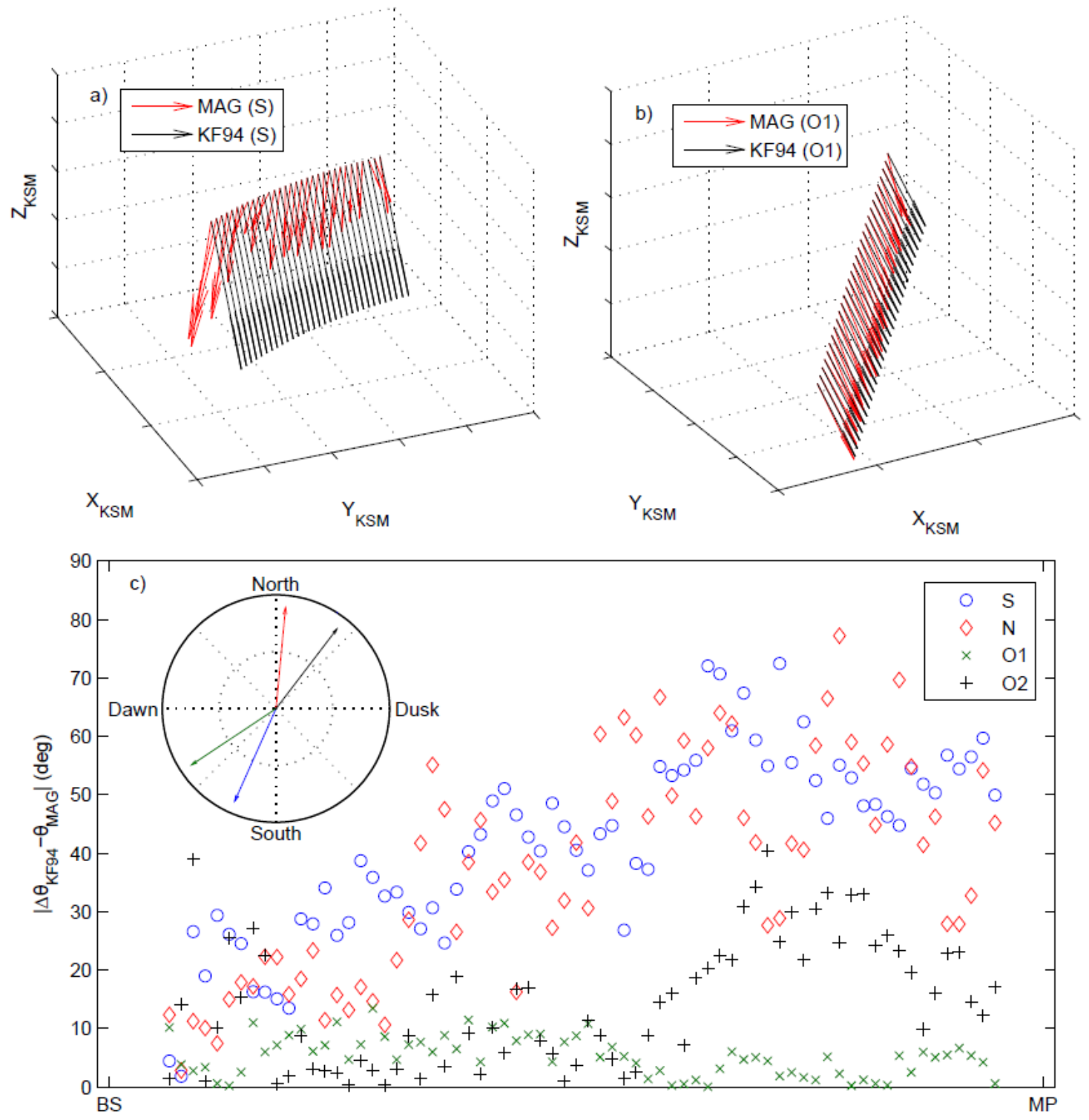

Figure 6.7.: Observed and KF94 predicted vector plots of draped field lines throughout magnetosheath traversal for a) near-southward and b) near-equatorial orientations. c) The angular difference between observed and KF94 for different IMF clock angles (inset). 


\subsection{Discussion}

Our results present the dayside draping of the observed magnetosheath, which were mainly originated as the Parker spiral configuration. We assessed the asymmetry of the field line curvatures using MHD simulations with the expectation that the asymmetry of the magnetopause will be manifested. Our analysis from observations were limited due to the lack of an upstream monitor to mitigate the effects of solar wind variability and boundary motion, both of which dictate Cassini's fractional position in the magnetosheath as well as restricting the model comparisons to (quasi-) steady state traversals.

The MHD simulations show a clear-cut asymmetry in the angular variations with longitude and latitude for IMF vectors aligned in the equatorial and meridional planes respectively. This revealed $\sim 15 \%$ more curvature from the angular gradients calculated in the meridional over the equatorial planes and this is indicative of the effect of the poles being more flattened than the flanks on the global magnetic structure. The MHD simulation is in broad agreement with measurements of the azimuthal angle of the magnetosheath across a wide range of longitudes by having a similar gradient to the distribution of observed azimuthal angles on the equatorial plane. The statistics for the meridional angle, on the other hand, are weak due to the lack of high latitude coverage as well as the very few incidents of steady northward or southward IMF orientations. The five events studied exhibit some trend compared to the MHD simulated sets although they are not statistically conclusive and there is some spread owing to their nonperfect alignment as well as some variability in the magnetosheath. See inset in Figure $6.7 \mathrm{c}$ for $\mathrm{S}, \mathrm{N}$ and $\mathrm{O} 2$ (three of the five events).

Nevertheless, despite the magnetic field lines being organised equatorially, the scattering of the observations in Figure 6.6 reveal the significance of nonzero meridional angles. This is also highlighted in Figure 6.3. The distributions of the meridional angles in both flanks show substantially more non-zero meridional angles compared with the Parker spiral upstream of Saturn [Jackman et al., 2008] which generally has a small $B_{z}$ component. Apart from the temporal variability of the solar wind, it is likely that the non-axisymmetry of Saturn's magne- 
tosphere is responsible for twisting the magnetic field out of the equatorial plane; consistent with the prediction by Erkaev et al. [1996] and Farrugia et al. [1998].

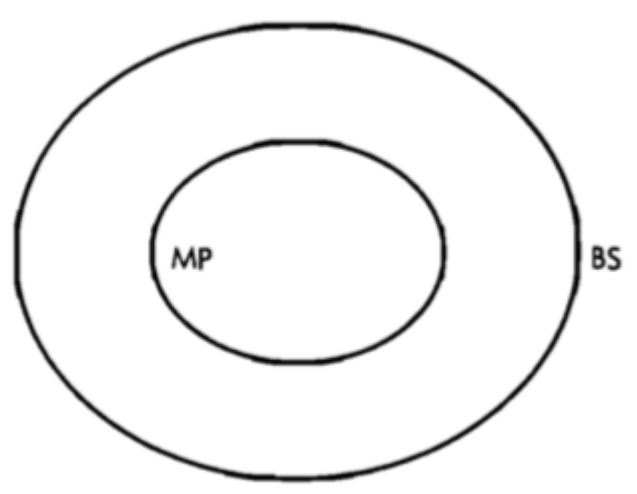

Figure 6.8.: A conceptual representation of the magnetopause (MP) and bow shock (BS) shapes of Saturn in the terminator plane. The ring bounded by the two ellipses is the magnetosheath. Adapted from Slavin et al. (1985), Licence \#3790801410531.

The higher $M_{A}$ regime at Saturn compared to Earth means that the dynamic pressure term (containing the flow speed $u$ ) is much more important than the magnetic pressure term (containing $\boldsymbol{B}$ ). The magnetic field lines in a sense are 'along for the ride' with the flow. In the context of momentum, the more important pressure gradient drives the flow as opposed to the relatively weaker $\boldsymbol{J} \times \boldsymbol{B}$ force (where $\boldsymbol{J}=1 / \mu_{0} \nabla \times \boldsymbol{B}$ ). This naturally makes the flow behaviour as the source of the field line twisting and this can be explained from Figure 6.8 which shows the polar-flattened magnetopause obstacle 'head-on' in the flow direction. The flow streamlines in this arrangement are non-axially symmetric with mass flux having to be transported along longer paths in the equatorial (broader) region and shorter paths in the polar (narrower) region. This would lead to preferential flow polar flow with the discrepancy increasing with degree of polar flattening. Thus a convected equatorial field line would experience a net torque and twist in the direction of the preferential flow leading to an increase in its $Z$ component.

Using KF94, we see better correspondence between the clock angles of the predicted and observed magnetic field vectors with a lower $B_{z}$ component. Longmore et al. [2006] conclude this is due to field-flow coupling effects in 
which the bulk flow acts on the magnetic field lines to twist the IMF. These contribute towards rotating the magnetic field lines in the direction of the accelerated flankward flow. Consistent with the study, a higher $B_{z}$ component leads to a rotation in the clock angle relative to KF94. However with limited coverage of the magnetosheath and plasma instruments, the role this plays in Saturn's magnetosheath cannot be corroborated. Here in particular, we find a more significant rotation for the higher $B_{z}$ compared to Earth and this could also possibly be due to the significant meridional confinement at Saturn. Thus the neglect of both field-flow coupling effects and boundary asymmetry lead to departures from the prediction and this is indicative of their role in the overall draping pattern. Nevertheless, KF94 has a potential reliability in determining the draping pattern and ultimately the shear angle near the magnetopause when Cassini is in the IMF region given that the orientation is near equatorial and assuming it will remain fairly steady. The Michigan Solar Wind Model (MSWiM) is commonly used to predict conditions of the solar wind upstream of Saturn. This is a one-dimensional (1-D) magnetohydrodynamic code that uses near-Earth spacecraft measurements as boundary conditions at $1 \mathrm{AU}$ and simulates the evolution of solar wind parameters along heliocentric distances through to 10 AU (beyond Saturn's orbit). Comparisons of the predicted and observed data are found to be most in agreement when the solar wind exhibited a recurrent pattern during the declining phase of the solar cycle. Furthermore, the speed propagations were most accurate within approximately \pm 75 days of apparent opposition (Sun-Earth-Saturn alignment taking into account transit time of the solar wind between Earth and Saturn) [Zieger and Hansen, 2008]. KF94 can be used in conjunction with propagations from MSWiM and remote auroral observations to investigate reconnection at the magnetopause when Cassini is in the IMF or magnetosphere during the aforementioned apparent opposition.

Future work will focus on the effect of the non-axisymmetry on the evolution of and twisting of the magnetic field lines out of the equatorial plane. It will be interesting to compare with works by Desroche et al., [2013]. More observations and specifically steady traversals will be investigated which could potentially support these findings. 


\section{Summary and Perspective}

This thesis utilises observations of the near-Saturn magnetic field environment to address questions related to very broad and well-established subjects; namely shock physics and field draping patterns. The longstanding interests of these areas are reflected in the literature which span decades. Detailed theoretical models and sophisticated computer simulations have been developed, both of which have immensely advanced our understanding of space plasmas. The work in this thesis builds on the strong foundations by taking advantage of the Cassini mission to present and interpret relevant observations that have not been presented either sufficiently or at all.

In Chapter 4, we show a distribution of the Alfvén Mach number $M_{A}$ and magnetic contact angle $\theta_{B n}$ of Saturn's dayside bow shock. Owing to both the weaker magnetic field and the Parker spiral at $10 \mathrm{AU}$, the shock is typically characterised as supercritical and quasi-perpendicular. The very wide range of $M_{A}$, across two orders of magnitude, means we are able to explore the evolution of magnetic overshoots and variability as a function of $M_{A}$ and $\theta_{B n}$. The overshoot was shown to strongly correlate with the $M_{A}$ and the view of the author is that the Mach number is indeed the primary controller.

This chapter also requires the use of a technique to determine the $M_{A}$ of each crossing and is used here for the first time. This uses dynamic pressure estimates from the W11 model and magnetic field measurements to bypass the need of operating plasma instruments and favourable pointing directions to determine arguably the most important plasma shock parameter, $M_{A}$. The development of an $M_{A}$ parameter space (Figure 4.6) has not only presented the most comprehensive picture of Saturn's bow shock but, more interestingly, has laid the groundwork for a new chapter in which the most exotic and rarest of shocks were explored. 
In Chapter 5, we use a combination of magnetic field and particle data (where available) to focus on the poorly-explored highest Mach numbers. Regular pulses in the magnetic field time series become more apparent with increasing $M_{A}$. When normalised to the upstream gyroperiod $\tau_{c i}$, the average periods of these pulses are $\sim 0.3 \tau_{c i}$ and consistent with the predicted timescale for specular reflection. These are interpreted as reformation cycles with specular reflection probably being the dominant process taking place. The crossings with these regular pulses or 'reformation cycles' are also shown to exhibit a noticeably larger maximum magnetic field at the overshoot than crossings without these features (for similar $\left.M_{A}\right)$.

In Chapter 6, we present a global overview of the magnetic field structure in Saturn's magnetosheath. The polar flattened magnetosphere is found be manifested in the overall draping pattern. This average draping pattern is well captured and in broad agreement for given upstream conditions with those of the MHD simulations. In comparison with an analytical model of axisymmetric geometry, deviations suggest that non-axisymmetry is indeed invariably a key feature of the magnetosphere's global structure. The main finding here is the significant rotation of the magnetic field out of the ecliptic plane which may be useful for models on reconnection at Saturn's magnetopause; since the magnetic shear angle is a key parameter.

The work in this thesis can have broader implications, particularly outside the Saturnian environment. Firstly, the technique of estimating $M_{A}$ and $\theta_{B n}$ can be used for non-terrestrial planets such as Mercury and Jupiter (with the MESSENGER and JUICE spacecraft respectively) where there are single/non-spinning spacecraft and possibly limited plasma observations. The technique only requires a magnetometer and a traditional bow shock model (as widely developed for decades).

Even broader, there might be scope for extrapolating the work here to exoplanets where in situ data is non-existent. Exo-planetary research is becoming increasingly popular and there have already been reports of exo-planets similar to ones in our solar system [e.g. Nichols, 2011]. For example, some useful 
knowledge of the flow and thus draping of magnetic field lines could be deduced from the rotation rate of a gaseous/Jupiter-like exoplanet. A very fast rotating gaseous exo-planet would expect to have a significant degree of polar flattening. Some conclusions could therefore be drawn about the momentum and energy transfer from the external stellar wind into its magnetosphere through reconnection, which depends on the magnetic shear angle as explained in Chapter 6. In addition, some estimate of the magnetic field strength just upstream of an exoplanet may provide knowledge on the nature and variability of a bow shock, though this may be more useful in the context of supernova remnants (e.g. particle acceleration and cosmic rays) as described in Chapter 5.

As for future work directly following this thesis, the $M_{A}$ parameter space can be further explored to investigate the evolution of quasi-parallel shocks with $M_{A}$. The main limitation here would be the much smaller sample of quasi-parallel shocks compared to quasi-perpendicular owing to the Parker spiral. For lower $M_{A}$ the use of the Radio and Plasma Wave Science (RPWS) instrument can be used to complement the magnetometer in investigating phase-locked whistler waves standing upstream of subcritical shocks. This could help identify the highlysought after whistler critical Mach number, $M_{w}$. Further work can also make use of more MHD runs for different upstream parameters to quantify the how the rotation evolves with increasing distance in the magnetosheath. The availability of high latitude orbits near the end of Cassini's mission could prove useful in corroborating and completing the picture of field line draping around nonaxisymmetric magnetospheres. 


\section{Bibliography}

Abraham-Shrauner, B. (1972), Determination of magnetohydrodynamic shock normal, J. Geophys. Res., 77,736, doi:10.1029/JA077i004p00736.

Achilleos, N., et al. (2006), Orientation, location, and velocity of Saturn's bow shock: Initial results from the Cassini spacecraft, J. Geophys. Res., 111, 3201, doi:10.1029/2005JA011297.

Achilleos, N., et al. (2008), Large-scale dynamics of Saturn's magnetopause:

Observations by Cassini, J. Geophys. Res., 113, A11209, doi:10.1029/2008JA013265.

Acuna, M. H., and N. F. Ness (1980), The magnetic field of Saturn - Pioneer 11 observations, Science, 207, 444-446, doi:10.1126/science.207.4429.444.

Alfvén, H. (1942), Existence of electromagnetic-hydromagnetic waves, Nature, 150, 405, doi:10.1038/150405d0.

Andrés, N., et al. (2013), Saturn's ULF wave foreshock boundary: Cassini observations, Planet. Space Sci., 79, 64-75, doi:10.1016/j.pss.2013.01.014.

Badman, S. V. and S. W. H. Cowley (2007), Significance of Dungey-cycle flows in Jupiter's and Saturn's magnetosphere, and their identification on closed equatorial field lines, Ann. Geophys., 25, 941-951, doi:10.5194/angeo-25-941-2007.

Bagenal, F., et al. (1987), The Uranian bow shock: Voyager 2 inbound observations of a high Mach number shock, J. Geophys Res., 92, 8603, doi:10.1029/JA092iA08p08603.

Bale, S. D., et al. (1998), Bipolar electrostatic structures in the shock transition region: Evidence of electron phase space holes, Geophys. Res. Lett., 25, 2929-2932, doi:10.1029/98GL02111. 
Bale, S. D., et al. (2003), Density-Transition Scale at Quasiperpendicular Collisionless Shocks, Phys. Rev. Lett., 91, 5001-5004, doi:10.1103/PhysRevLett.91.265004.

Balikhin, M. and M. Gedalin (1994), Kinematic mechanism of electron heating in shocks: Theory vs observations, Geophys. Res. Lett., 21, 841-844, doi:10.1029/94GL00371.

Balogh, A., et al. (1992), The magnetic field investigation on the ULYSSES mission - Instrumentation and preliminary scientific results, Astron. Astrophys., 92, 221-236.

Baumjohann, W., and R. A. Treumann (1997), Basic Space Plasma Physics, Imperial College Press.

Belcher, J. W. (1973), A variation of the Davis-Smith method for in-flight determination of spacecraft magnetic fields, J. Geophys. Res., 78, 6840-6490, doi:10.1029/JA078i028p06480.

Bertucci, C., et al. (2007), Low-frequency waves in the foreshock of Saturn: First results from Cassini, J. Geophys. Res., 112, 9219, doi:10.1029/2006JA012098.

Bertucci, C., et al. (2015), Titan's interaction with the supersonic solar wind, Geophys. Res. Lett., 42, 193-200, doi:10.1002/2014GL062106.

Billig, F. S. (1967), Shock-wave shapes around spherical-and cylindrical-nosed bodies, Journal of Spacecraft and Rockets, 4, 822-823, doi:10.2514/3.28969.

Biskamp, D., and H. Welter (1972), Numerical studies of magnetosonic collisionless shock waves, Nucl. Fusion, 12, 663, doi:10.1088/0029-5515/12/6/006.

Bondi, H., and T. Gold (1950), On the generation of magnetism by fluid motion, Mon. Not. R. Astron. Soc., 110, 607-611, doi:10.1093/mnras/110.6.607.

Boyd, T. J. M., and J. J. Sanderson (2003), The Physics of Plasmas, Cambridge University Press, Cambridge.

Bridge, H. S., et al. (1982), Plasma observations near Saturn - Initial results from Voyager 2, Science, 215, 563-570, doi:10.1126/science.215.4532.563. 
Bunce, E. J. et al., (2008), Origin of Saturn's aurora: Simultaneous observations by Cassini and the Hubble Space Telescope, J. Geophys. Res., 113, A09209, doi:10.1029/2008JA013257.

Burgess, D. (1995), Collisionless Shocks, in Introduction to Space Physics, edited by M. G. Kivelson and C. T. Russell, Cambridge University Press, Cambridge.

Burgess, D. (2006), Interpreting multipoint observations of substructure at the quasiperpendicular bow shock: Simulations, J. Geophys. Res., 111, 10210, doi:10.1029/2006JA011691.

Burton, M. E., et al. (2001), The Cassini/Huygens Venus and earth flybys: An overview of operations and results, J. Geophys. Res., 106, 30099-30108, doi:10.1029/2001JA900088.

Chapman, S. C., et al. (2005), Perpendicular shock refomraion and ion reflection, Space Sci.Rev., 121, 5, doi:10.1007/s11214-006-4481-x.

Chapman, S., and V. C. A. Ferraro (1930), A new theory of magnetic storms, Nature, 126, 129-130.

Comisel, H., et al. (2011), Non-stationarity of the quasi-perpendicular bow shock: comparison between Cluster observations and simulations, Ann. Geophys., 29, 263, doi:10.5194/angeo-29-263-2011.

Cooling, B. M. A., et al. (2001), Role of the magnetosheath flow in determining the motion of open flux tubes, J. Geophys. Res., 106, 18763-18776, doi:10.1029/2000JA000455.

Crary, F. J., et al. (2005), Solar wind dynamic pressure and electric field as the main factors controlling Saturn's aurorae, Nature, 433, 720-722, doi:10.1038/nature03333.

Crooker, N. U., et al. (1985), Magnetic field draping against the dayside magnetopause, J. Geophys. Res., 90, 3505-3510, doi:10.1029/JA090iA04p03505.

de Hoffmann, F. and E. Teller (1950), Magneto-hydrodynamic Shocks, Phys. Rev., 80, 692-703, doi:10.1103/PhysRev.80.692. 
Debye, P., and E. Hückel (1923), On the Debye length in strong electrolytes, Physikal.Z., 24(9), 185-206.

Desroche, M., et al. (2013), Conditions at the magnetopause of Saturn and implications for the solar wind interaction, J. Geophys. Res., 118, 3087- 3095, doi10.1002/jgra.50294.

DiBraccio, G. A., et al. (2013), MESSENGER observations of magnetopause structure and dynamics at Mercury, J. Geophys. Res., 118, 997-1008, doi:10.1002/jgra.50123.

Dougherty, M. K., et al. (2004), The Cassini magnetic field investigation, Space Sci. Rev., 114(1-4), 331-383, doi:10.1007/s11214-004-1432-2.

Dougherty, M. K., et al. (2006), Identification of a dynamic atmosphere at Enceladus with the Cassini magnetometer, Science, 311, 1406-1409, doi:10.1126/science.1120985.

Dungey, J. W. (1961), Interplanetary magnetic field and the auroral zones, Phys. Rev. Lett., 6, 47-48, doi:10.1103/PhysRevLett.6.47.

Dunlop, M. W. (1999), Operation of the dual magnetometer on Cassini: science performance, Planet. Space Sci., 47, 1389-1405, doi:10.1016/S0032-0633(99)000604.

Erkaev, N. V., et al. (1996), Effects on the Jovian magnetosheath arising from solar wind flow around nonaxisymmetric bodies, J. Geophys. Res., 101, 10,665-10,672, doi:10.1029/95JA03518.

Erkaev, N. V., et al. (2011), On accelerated magnetosheath flows under northward IMF, Geophys. Res. Lett., 38, L01104, doi:10.1029/2010GL045998.

Erkaev, N. V., et al. (2012), Accelerated magnetosheath flows caused by IMF draping: Dependence on latitude, Geophys. Res. Lett., 39, L01103, doi:10.1029/2011GL050209.

Espinosa, S. A., and M. K. Dougherty (2000), Periodic perturbations in Saturn's magnetic field, Geophys. Res. Lett., 27, 2785-2788, doi:10.1029/2000GL000048. 
Fairfield, D. H. (1967), The ordered magnetic Field of the magnetosheath, $J$. Geophys. Res., 72, 5865-5877, doi:10.1029/JZ072i023p05865.

Farrugia, C. J., et al. (1998), The effect of the magnetopause shapes of Jupiter and Saturn on magnetosheath parameters, Planet. Space Sci., 46, 507-514, doi:10.1016/S0032- 0633(97)00225-0.

Formisano, V. (1979), Orientation and shape of the earth's bow shock in three dimensions, Planet. Space Sci.Rev., 27, 1151-1161, doi:10.1016/00320633(79)90135-1.

Gershman, D. J., et al. (2013), Magnetic flux pileup and plasma depletion in Mercury's subsolar magnetosheath., J. Geophys. Res., 118, 7181-7199, doi:10.1002/2013JA019244.

Ghavamian, P., et al. (2013), Electron-Ion Temperature Equilibration in Collisionless Shocks: The Supernova Remnant-Solar Wind Connection, Space Sci.Rev., 178, 633663, doi:10.1007/s11214-013-9999-0.

Goldstein, M. L., et al. (1995), Magnetohydrodynamic turbulence in the solar wind, Astron. Astrophys., 33, 283, doi:10.1146/annurev.aa.33.090195.001435.

Gombosi, T. I., et al. (2002), Semi-relativistic magnetohydrodynamics and physics based convergence acceleration, J. Comput. Phys., 177, 176-205, doi:10.1006/jeph.2002.7009.

Gosling, J. T., and M. F. Thomsen (1985), Specularly reflected ions, shock foot thicknesses, and shock velocity determinations in space, J. Geophys. Res., 90, A10, doi:10.1029/JA090iA10p09893.

Gringauz, K. I., et al. (1960), Study of the interplanetary high energy electrons and solar corpuscular radiation by means of three electrode traps for charged particles on the second Soviet cosmic rocket, Soviet Phys. Doklady, 53, 61.

Guo, F., and J. Giacalone (2015), The Acceleration of Electrons at Collisionless Shocks Moving Through a Turbulent Magnetic Field, Astrophys. J., 802, 97, doi:10.1088/0004-637X/802/2/97. 
Gurnett, D. A., et al. (2004), The Cassini radio and plasma wave investigation, Space Sci.Rev., 114(1-4), 395-463, doi:10.1007/s11214-004-1434-0.

Gurnett, D. A., et al. (2013), In situ observations of interstellar plasma with voyager 1, Science, 341, 1489-1492, doi:10.1126/science.1241681.

Hellinger, P., et al. (2002), Reformation of perpendicular shocks: Hybrid simulations, Geophys. Res. Lett., 29, 2234, doi:10.1029/2002GL015915.

Hendricks, S., et al. (2005), Variability in Saturn's bow shock and magnetopause from Pioneer and Voyager: Probabilistic predictions and initial observations by Cassini, Geophys. Res. Lett., 32, L20S08, doi:10.1029/2005GL022569.

Henley, E. M. (2010), Scales and variability of Earth's bow shock, PhD Thesis, Imperial College London.

Hill, T. W. (1979), Inertial limit on corotation, J. Geophys. Res., 84, 6554-6558, doi:10.1029/JA084iA11p06554.

Horbury, T. S., et al. (2001), Cluster magnetic field observations of the bowshock: Orientation, motion and structure, Ann. Geophys., 19, 1399, doi:10.5194/angeo-191399-2001.

Hundhausen, A. J. (1995), The Solar Wind, in Introduction to Space Physics, edited by M. G. Kivelson and C. T. Russell, Cambridge University Press, Cambridge.

Jackman, C. M., and C. S. Arridge (2011), Solar Cycle Effects on the Dynamics of Jupiter's and Saturn's Magnetospheres, Solar Phys., 274, 481-502, doi:10.1002/2014GL059288.

Jackman, C. M., et al. (2007), Strong rapid dipolarizations in Saturn's magnetotail: In situ evidence of reconnection, Geophys. Res. Lett., 34, L11203, doi:10.1029/2007GL029764.

Jackman, C. M., et al. (2008), The overall configuration of the interplanetary magnetic field upstream of Saturn as revealed by Cassini observations, J. Geophys. Res., 113, 8114, doi:10.1029/2008JA013083. 
Jia, X., et al. (2012), Magnetospheric configuration and dynamics of Saturn's magnetosphere: A global MHD simulation, J. Geophys. Res., 117, A05225, doi:10.1029/2012JA017575.

Kanani, S. J., et al. (2010), A new form of Saturn's magnetopause using a dynamic pressure balance model, based on in situ, multi-instrument Cassini measurements, $J$. Geophys. Res., 115, A06207, doi:10.1029/2009JA014262.

Kellock, S., et al. (1996), Cassini dual technique magnetometer instrument (MAG), SPIE, 2803. 141-152.

Kivelson, M. G. (1995), Physics of Space Plasmas, in Introduction to Space Physics, edited by M. G. Kivelson and C. T. Russell, Cambridge University Press, Cambridge.

Kivelson, M. G. and D. J. Southwood (2005), Dynamical consequences of two modes of centrifugal instability in Jupiter's outer magnetosphere, J. Geophys. Res., 110, A9, doi:10.1029/2005JA011176.

Klein, L. E., and L. F. Burlaga (1982), Interplanetary magnetic clouds at 1 AU, J. Geophys. Res., 87, 613-624, doi:10.1029/JA087iA02p00613.

Kobel, E., and Flückiger, E. O. (1994), A model of the steady state magnetic field in the magnetosheath, J. Geophys. Res., 99, 23617, doi:10.1029/94JA01778.

Krasnoselskikh, V. V., et al. (2002), Nonstationarity of strong collisionless quasiperpendicular shocks: Theory and full particle numerical simulations, Phys. Plasmas, 9, 1192, doi:10.1063/1.1457465.

Landau, L. D., and E. M. Lifshitz (1959), Fluid Mechanics, pp. 310-313, Pergamon, New York.

Lembege, B., and J. M. Dawson (1987), Plasma heating through a supercritical oblique collisionless shock, Phys. Fluids, 30, 1110, doi:10.1063/1.866309.

Lepping, R. P., et al. (1981), Surface waves on Saturn's magnetopause, Nature, 292, 750-753, doi:10.1038/292750a0. 
Leroy, M. M., et al. (1981), Simulation of a perpendicular bow shock, Geophys. Res. Lett., 8, 1269-1272, doi:10.1029/GL008i012p01269.

Leroy, M. M., et al. (1982), The structure of perpendicular bow shocks, J. Geophys Res., 87, A7, doi:10.1029/JA087iA07p05081.

Lobzin, V. V., et al. (2007), Nonstationarity and reformation of high-Mach-number quasiperpendicular shocks: Cluster observations, Geophys. Res. Lett., 34, L05107, doi:10.1029/2006GL029095.

Longmore, M., et al. (2006), Rotation of the magnetic field in Earth's magnetosheath by bulk magnetosheath plasma flow, Ann. Geophys., 24, 339-354, doi:10.5194/angeo-24-339-2006.

Marshall, W., (1955), The structure of magneto-hydrodynamic shock waves, Proc. R. Soc. Lond. A., 233, 367-376, doi:10.1098/rspa.1955.0272.

Masters, A., et al. (2008), An empirical model of Saturn's bow shock: Cassini observations of shock location and shape, J. Geophys. Res., 113, 10210, doi:10.1029/2008JA013276.

Masters, A., et al. (2011), Electron heating at Saturn's bow shock, J. Geophys. Res., 116, 10107, doi:10.1029/2011JA016941.

Masters, A., et al. (2012), The importance of plasma $\beta$ conditions for magnetic reconnection at Saturn's magnetopause, Geophys. Res. Lett., 39, L08103, doi:10.1029/2012GL051372.

Masters, A., et al. (2013), Electron acceleration to relativistic energies at a strong quasi-parallel shock wave, Nature Physics, 9, 164, doi:10.1038/nphys2541.

Masters, A., et al. (2014), The plasma depletion layer in Saturn's magnetosheath, $J$. Geophys. Res., 119, 121-130, doi:10.1002/2013JA019516.

Mistry, R., et al. (2014), Separating drivers of Saturnian magnetopause motion, $J$. Geophys. Res., 119, 1514-1522, doi: 10.1002/2013JA019489. 
Mitchell, J. J., and S. J. Schwartz (2013), Nonlocal electron heating at the Earth's bow shock and the role of the magnetically tangent point, 118, J. Geophys. Res., 7566-7575, doi:10.1002/2013JA019226.

Morse, D. L., et al. (1972), Nonstationarity behavior of collisionless shocks, Phys Rev. Lett., 28, 13, doi:10.1103/PhysRevLett.28.13.

Morton, R. J., et al. (2012), Observations of ubiquitous compressive waves in the Sun's chromosphere, Nat. Commun., 3, 1315, doi:10.1038/ncomms2324.

Murray, C. D., et al. (2008), The determination of the structure of Saturn's F ring by nearby moonlets, Nature, 453, 739-744, doi:10.1038/nature06999.

Ness, N. F., et al. (1981), Magnetic field studies by Voyager 1 - Preliminary results at Saturn, Science, 212, 211-217, doi:10.1126/science.212.4491.211.

Newbury, J. A., and C. T. Russell (1996), Observations of a very thin collisionless shock, Geophys. Res. Lett., 23, 781, doi:10.1029/96GL00700.

Nichols, J. D. (2011), Magnetosphere-ionosphere coupling at Jupiter-like exoplanets with internal plasma sources: implications for detectability of auroral radio emissions, Mon. Not. R. Astron. Soc., 414, 2125-2138, doi:10.1111/j.13652966.2011.18528.x.

O'Donoghue, J., et al. (2013), The domination of Saturn's low-latitude ionosphere by ring 'rain', Nature, 496, 193-195, doi:10.1038/nature12049.

Parker, E. N. (1958), Dynamics of the interplanetary gas and magnetic fields, Astrophys. J., 128, 664-676, doi: 10.1086/146579.

Paschmann, G. (2008), Recent in-situ observations of magnetic reconnection in nearEarth space, Geophys. Res. Lett., 35, 19109, doi:10.1029/2008GL035297.

Peredo, M., et al. (1995), Three-dimensional position and shape of the bow shock and their variation with Alfvenic, sonic and magnetosonic Mach numbers and interplanetary magnetic field orientation, J. Geophys. Res., 100, 7907-7916, doi:10.1029/94JA02545. 
Petrinec, S. M. (2012), On the magnetic field configutation of the magnetosheath, Terr. Atmos. Ocean. Sci., 24, 265-272, doi:10.3319/TAO.2012.10.17.02(SEC).

Pokhotelov, D., et al. (2013), Ion distributions upstream and downstream of the Earth's bow shock: first results from Vlasiator, Ann. Geophys., 31, 2207-2212, doi: 10.5194/angeo-31-2207-2013.

Porco, C. C., et al. (2005), Imagine of Titan from the Cassini spacecraft, Nature, 434, 159-168, doi:10.1038/nature03436.

Quest, K. B. (1985), Simulations of high-Mach-number collisionless perpendicular shocks in astrophysical plasmas, Phys. Rev. Lett., 54, 16, doi:10.1103/PhysRevLett.54.1872.

Richardson, J. D. (2002), The magnetosheaths of the outer planets, Planet. Space Sci., 50, 503-517, doi:10.1016/S0032-0633(02)00029-6.

Russell, C. T. (1976), Reconnection in physics of solar planetary environments, edited by D. J. Williams, pp. 526-540, American Geophysical Union, Washington D.C.

Russell, C. T., and E. W. Greenstadt (1979), Initial ISEE magnetometer results Shock observation, Space Sci.Rev., 23, 3-37, doi:10.1007/BF00174109.

Russell, C. T., et al. (1979), Initial Pioneer Venus magnetic field results - Dayside observations, Science, 203, 745-748, doi:10.1126/science.203.4382.745.

Russell, C. T., et al. (1982), Overshoots in planetary bow shocks, Nature, 296, 4548, doi:10.1038/296045a0.

Rymer, A. M., et al. (2013), Saturn's magnetospheric refresh rate, Geophys. Res. Lett., 40, 2479-2483, doi:10.1002/grl.50530.

Scholer, M., et al. (2003), Quasi-perpendicular shocks: Length scale of the crossshock potential, shock reformation, and implication for shock surfing, J. Geophys. Res., 108, 1014, doi:10.1029/2002JA009515. 
Scholer, M. and D. Burgess (2006), Transition scale at quasiperpendicular collisionless shocks: Full particle electromagnetic simulations, Phys. Plasmas, 13, 062101, doi:10.1063/1.2207126.

Scholer, M. and D. Burgess (2007), Whistler waves, core ion heating, and nonstationarity in oblique collisionless shocks, Phys. Plasmas, 14(1), 012108012109, doi:10.1063/1.2748391.

Scholer, M., and S. Matsukiyo (2004), Nonstationarity of quasi-perpendicular shocks: a comparison of full particle simulations with different ion to electron mass ratio, Ann. Geophys., 22, 2345, doi:10.5194/angeo-22-2345-2004.

Schwartz, S. J. and D. Burgess (1991), Quasi-parallel shocks - A patchwork of threedimensional structures, Geophys. Res. Lett., 18, 373-376, doi:10.1029/91GL00138.

Schwartz, S. J., et al. (2002), Astrophysical Plasmas, Postgraduate course, Queen Mary University of London.

Sckopke, N., et al. (1983), Evolution of ion distributions across the nearly perpendicular bow shock - Specularly and non-specularly reflected-gyrating ions, $J$. Geophys. Res., 88, 6121-6136, doi:10.1029/JA088iA08p06121.

Slavin, J. A., et al. (1983), A Pioneer-Voyager study of the solar wind interaction with Saturn, Geophys. Res. Lett., 10, 9-12, doi:10.1029/GL010i001p00009.

Slavin, J. A., et al. (1984), Planetary Mach cones - Theory and observations, $J$. Geophys. Res., 89, 2708-2714, doi:10.1029/JA089iA05p02708.

Slavin, J. A., et al. (1985), Solar wind flow about the outer planets - Gas dynamic modeling of the Jupiter and Saturn bow shocks, J. Geophys. Res., 90, 6275-6286, doi:10.1029/JA090iA07p06275.

Slavin, J. A., et al. (2009), MESSENGER observations of magnetic reconnection in Mercury's magnetosphere, Science, 324, 606-610, doi:10.1126/science.1172011.

Smith, B. A., et al. (1982), A new look at the Saturn system - The Voyager 2 images, Science, 215, 504-537, doi:10.1126/science.215.4532.504. 
Snyder, C. W., and M. Neugebaur (1966), The relation of Mariner-2 plasma data to solar phenomena, in The Solar Wind, pp. 25-32, Oxford: Permanon Press.

Sonnerup, B. U. O. (1974), Magnetopause reconnection rate, J. Geophys. Res., 79, 1546-1549, doi:10.1029/JA079i010p01546.

Southwood, D. J. and M. G. Kivelson (1989), Magnetospheric interchange motions, J. Geophys. Res., 94, 299-307, doi:10.1029/JA094iA01p00299.

Southwood, D. J. and M. G. Kivelson (1993), Mirror instability. I - Physical mechanism of linear instability, J. Geophys. Res., 98, 9181-9187, doi:10.1029/92JA02837.

Spreiter, J. R., and S. S. Stahara (1980), A new predictive model for determining solar wind-terrestrial planet interactions, J. Geophys. Res., 85, A12, 6769-6777, doi:10.1029/JA085iA12p06769.

Spreiter, J. R., et al. (1966), Hydrodynamic flow around the magnetosphere, Planet. Space Sci., 14, 223-253, doi:10.1016/0032-0633(66)90124-3.

Stevenson, D. J. (1982), Formation of the giant planets, Planet. Space Sci., 30, 755764, doi:10.1016/0032-0633(82)90108-8.

Thomsen, M. F., et al. (1987), On the noncoplanarity of the magnetic field within a fast collinsionless shock, J. Geophys. Res., 92, 2305-2314, doi:10.1029/JA092iA03p02305.

Thomsen, M. F., et al. (2013), Saturn's magnetospheric dynamics, Geophys. Res. Lett., 40, 5337-5344, doi:10.1002/2013GL057967.

Tiu, D., et al. (2011), Evidence for reformation of the Uranian bow shock: Hybrid simulations and comparisons with Voyager data, J. Geophys. Res., 116, A04228, doi:10.1029/2010JA016057.

Tonks, L., and I. Langmuir (1929), Oscillations in ionized gases, Phys. Rev., 33, 195-210. 
Treumann, R. A. (2009), Fundamentals of collisionless shocks for astrophysical application, 1. Non-relativistic shocks, Astron. Astrophys. Rev., 17, 409-535, doi:10.1007/s00159-009-0024-2.

Vasyliunas, V. M. (1983), Plasma distribution and flow, in Physics of the Jovian magnetosphere, pp. 395-453.

Violante, L., et al. (1995), Observations of mirror mode waves and plasma depletion layer upstream of Saturn's magnetopause, J. Geophys. Res., 100, A7, 12047-12055, doi:10.1029/94JA02703.

Vlasov, A. A. (1968), The vibrational properties of an electron gas, Soviet Physics Uspekhi, 10, 721, doi:10.1070/PU1968v010n06ABEH003709.

Went, D. R., et al. (2011), A new semi-empirical model of Saturn's bow shock based on propagated solar wind parameters, J. Geophys. Res., 116, A07202, doi:10.1029/2010JA016349.

Winske, D. and N. Omidi (1996), A nonspecialist's guide to kinetic simulations of space plasmas, J. Geophys. Res., 101, 17289-17304, doi:10.1029/96JA00982.

Young, D. T., et al. (2004), Cassini plasma spectrometer investigation, Space Sci. Rev., 114(1-4), 1-112, doi:10.1007/s11214-004-1406-4.

Yuan, X., et al. (2009), Confirmation of quasi-perpendicular shock reformation in two-dimensional hybrid simulations, Geophy Res. Lett., 36, 5, doi:10.1029/2008GL036675.

Zieger, B., and Hansen, K. C. (2008), Statistical validation of a solar wind propagation model from 1 to $10 \mathrm{AU}$, J. Geophys. Res., 113, A08107, doi:10.1029/2008JA013046.

Zwan, B. J., and R. A. Wolf (1976), Depletion of solar wind plasma near a planetary boundary, J. Geophys. Res., 81, 1636-1648, doi:10.1029/JA081i010p01636. 